DOI 10.4171/JEMS/261

Thomas Duyckaerts · Carlos Kenig · Frank Merle

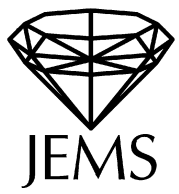

\title{
Universality of blow-up profile for small radial type II blow-up solutions of the energy-critical wave equation
}

Received August 1, 2009 and in revised form September 17, 2009

\begin{abstract}
Consider the energy-critical focusing wave equation on the Euclidian space. A blow-up type II solution of this equation is a solution which has finite time of existence but stays bounded in the energy space. The aim of this work is to exhibit universal properties of such solutions.

Let $W$ be the unique radial positive stationary solution of the equation. Our main result is that in dimension 3, under an appropriate smallness assumption, any type II blow-up radial solution is essentially the sum of a rescaled $W$ concentrating at the origin and a small remainder which is continuous with respect to the time variable in the energy space. This is coherent with the solutions constructed by Krieger, Schlag and Tataru. One ingredient of our proof is that the unique radial solution which is compact up to scaling is equal to $W$ up to symmetries.
\end{abstract}

\section{Contents}

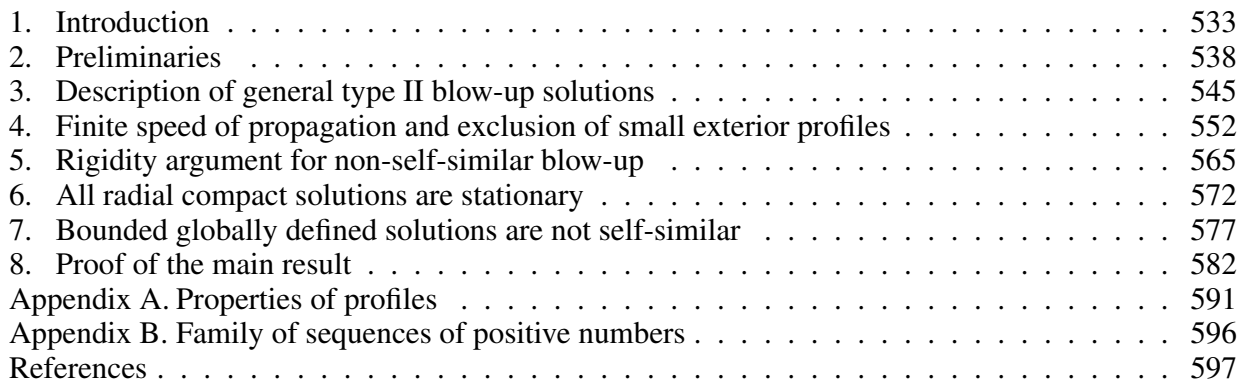

\section{Introduction}

Consider the focusing energy-critical wave equation on an interval $I(0 \in I)$,

$$
\left\{\begin{array}{l}
\partial_{t}^{2} u-\Delta u-|u|^{\frac{4}{N-2}} u=0, \quad(t, x) \in I \times \mathbb{R}^{N}, \\
u_{\uparrow t=0}=u_{0} \in \dot{H}^{1}, \quad \partial_{t} u_{\uparrow t=0}=u_{1} \in L^{2},
\end{array}\right.
$$

T. Duyckaerts, F. Merle: Département de mathématiques, Université de Cergy-Pontoise, Site de Saint Martin, 2, avenue Adolphe Chauvin, 95302 Cergy-Pontoise Cedex, France; e-mail: tduyckae@u-cergy.fr, frank.merle@u-cergy.fr

C. Kenig: Department of Mathematics, University of Chicago, 5734 University Avenue,

Chicago, IL 60637-1514, USA; e-mail: cek@ math.uchicago.edu 
where $u$ is real-valued, $N \in\{3,4,5\}$, and $\dot{H}^{1}:=\dot{H}^{1}\left(\mathbb{R}^{N}\right)$. We set $S(I):=$ $L^{\frac{2(N+1)}{N-2}}\left(I \times \mathbb{R}^{N}\right)$. We will often restrict ourselves to the case of radial solutions in space dimension $N=3$.

The Cauchy problem (1.1) is locally well-posed in $\dot{H}^{1} \times L^{2}$. This space is invariant under the scaling of the equation: if $u$ is a solution to [1.1], $\lambda>0$ and

$$
u_{\lambda}=\frac{1}{\lambda^{(N-2) / 2}} u\left(\frac{t}{\lambda}, \frac{x}{\lambda}\right)
$$

then $u_{\lambda}$ is also a solution and $\left\|u_{\lambda}(0)\right\|_{\dot{H}^{1}}=\left\|u_{0}\right\|_{\dot{H}^{1}},\left\|\partial_{t} u_{\lambda}(0)\right\|_{L^{2}}=\left\|u_{1}\right\|_{L^{2}}$.

Let $T_{+} \in(0,+\infty]$ be the maximal positive time of definition for the solution $u$. It satisfies the following finite time blow-up criterion:

$$
T_{+}<\infty \Rightarrow\|u\|_{S\left(0, T_{+}\right)}=\infty .
$$

Note that this criterion does not rule out type II blow-up, i.e. solutions such that $T_{+}<\infty$ and

$$
\sup _{t \in\left[0, T_{+}\right)}\left[\left\|\partial_{t} u(t)\right\|_{L^{2}}^{2}+\|\nabla u(t)\|_{L^{2}}^{2}\right]<\infty .
$$

This is different from the case of lower order nonlinearity (of the form $|u|^{p-1} u$ with $p<(N+2) /(N-2))$, where the finite time blow-up implies the blow-up of the energy norm.

Energy arguments of Levine type [Lev74] are not expected to give directly type II blow-up. Examples of radial type II blow-up solutions of (1.1) were constructed in space dimension $N=3$ by Krieger, Schlag and Tataru [KST09]. The aim of this article is to exhibit universal properties of this type of solutions.

Let

$$
W:=\frac{1}{\left(1+\frac{|x|^{2}}{N(N-2)}\right)^{(N-2) / 2}},
$$

which is a stationary solution of [1.1]. The construction of [KST09] relies on an elaborate fixed point argument which yields the following description of the solution:

$$
u(t)=\frac{1}{\lambda(t)^{1 / 2}} W\left(\frac{x}{\lambda(t)}\right)+\varepsilon(t),
$$

where $\lambda(t)=\left(T_{+}-t\right)^{1+v}, v>0$ and

$$
\lim _{t \rightarrow T_{+}} \int_{|x| \leq T_{+}-t}|\nabla \varepsilon(t)|^{2} d x+\int_{|x| \leq T_{+}-t}\left|\partial_{t} \varepsilon(t)\right|^{2} d x+\int_{|x| \leq T_{+}-t}|\varepsilon(t)|^{6} d x=0 .
$$

In this work, we investigate the converse problem: if we consider an arbitrary type II radial blow-up solution, does such a decomposition hold?

We will obtain this result under an appropriate smallness assumption. From [KM08] (see also [DKM] $)$ if $N=3,4$ or $u$ is radial, and

$$
\sup _{t \in\left[0, T_{+}\right)}\left[\|\nabla u(t)\|_{L^{2}}^{2}+\left\|\partial_{t} u(t)\right\|_{L^{2}}^{2}\right]<\|\nabla W\|_{L^{2}}^{2},
$$


then $T_{+}=\infty$ and the solution scatters forward in time, and in particular does not blow up. In this work, the authors introduce a general road map to tackle such critical problems in focusing and defocusing situations. From a concentration-compactness result (in this case [BG99]), one reduces the proof to some rigidity property of solutions of (1.1] that are compact in the energy space up to the invariances of the equation. This rigidity property has to be shown by independent arguments.

The threshold $\|\nabla W\|_{L^{2}}^{2}$ is sharp. Indeed, from [KST09], for all $\eta_{0}>0$ there exists a type II blow-up solution such that

$$
\sup _{t \in\left[0, T_{+}\right)}\left[\|\nabla u(t)\|_{L^{2}}^{2}+\left\|\partial_{t} u(t)\right\|_{L^{2}}^{2}\right] \leq\|\nabla W\|_{L^{2}}^{2}+\eta_{0} .
$$

In the present article, we consider type II blow-up solutions such that 1.7 holds. Our main result is the following.

Theorem 1. Assume that $N=3$. There exists $\eta_{0}>0$ such that for any radial solution $u$ of (1.1) such that $T_{+}(u)=T_{+}<\infty$ that satisfies (1.7), there exist a solution $v(t)$ of (1.1) defined in a neighborhood of $t=T_{+}$, a sign $\iota_{0} \in\{ \pm 1\}$, and a positive $C^{0}$ function $\lambda(t)$ on $\left(0, T_{+}\right)$such that, as $t \stackrel{<}{\rightarrow} T_{+}$,

$$
\begin{aligned}
u(t) & =v(t)+\frac{\iota_{0}}{\lambda(t)^{1 / 2}} W\left(\frac{x}{\lambda(t)}\right)+o(1) \quad \text { in } \dot{H}^{1}, \\
\partial_{t} u(t) & =\partial_{t} v(t)+o(1) \quad \text { in } L^{2} \\
\lambda(t) & =o\left(T_{+}-t\right) .
\end{aligned}
$$

Note that 1.8, 1.9) imply that $u$ is of the form 1.5) with $\varepsilon$ satisfying (1.6): any radial blow-up solution satisfying (1.7) is of the type of the solutions constructed by Krieger, Schlag and Tataru.

As is now well-known from previous works on similar problems (see remarks below), the result is based on classification of solutions of (1.1) that are compact up to the symmetry of the equation. We state this result for its own interest.

Theorem 2. Let u be a nonzero radial solution of (1.1) in space dimension $N=\{3,4,5\}$. Assume that there exists a function $\lambda(t)$ of $t \in\left(T_{-}(u), T_{+}(u)\right)$ such that

$$
K=\left\{\left(\lambda(t)^{N / 2-1} u(t, \lambda(t) \cdot), \lambda(t)^{N / 2} \partial_{t} u(t, \lambda(t) \cdot)\right): t \in \mathbb{R}\right\}
$$

has compact closure in $\dot{H}^{1} \times L^{2}$. Then there exist $\lambda_{0}>0$ and a sign $\iota_{0} \in\{ \pm 1\}$ such that

$$
u(t, x)=\frac{\iota_{0}}{\lambda_{0}^{N / 2-1}} W\left(\frac{x}{\lambda_{0}}\right) .
$$

Remark 1.1. The proof of Theorem 2 (see Section 6) uses the material of [DM08], where a first classification result of this type was obtained. Namely, at the energy threshold $E\left(u_{0}, u_{1}\right)=E(W, 0)$, all solutions such that $\int\left|\nabla u_{0}\right|^{2}+\int\left|u_{1}\right|^{2} \leq \int|\nabla W|^{2}$ are globally defined, and the only ones that do not scatter are (up to the transformations of the equation) $W$ and a solution $W^{-}$, which scatters backward in time and tends to $W$ exponentially as $t$ goes to $\infty$. 
Remark 1.2. These results are essential to understand type II blow-up. After one has exhibited a universal profile for blow-up, one can hope using local dynamics near $W$, or linearization around $W$ (see e.g. [KS07]) to understand the possible blow-up speeds, which will complete the program to understand type II blow-up. Moreover, this is the first step to prove that the boundary of the set of initial data that lead to blow-up is given by type II blow-up solutions.

The proof of Theorem 1 highlights, through the mechanism of profile decomposition and the finite speed of propagation, why the only candidates to be type II blow-up profiles are compact solutions. The only case where such a striking fact was established was for GKdV by Martel and Merle [MM00, MM01].

Remark 1.3. In the case of nonlinear wave maps, all blow-up solutions are of type II: the equation is defocusing, in the sense that the energy provides a bound on the energy norm. An analogue of Theorem 1 is known locally in space for a sequence of times (without size condition due to the defocusing nature of the equation). Namely, if $u$ is a blow-up solution, there exist a sequence of times $t_{n} \rightarrow T_{+}$and a sequence $\lambda_{n} \rightarrow 0^{+}$such that $u\left(t_{n}, x / \lambda_{n}\right)$ tends to a nonlinear object. This follows from a remarkable paper of Christodoulou and Tahvildar-Zadeh [CTZ93]. See also the article of Shatah and Tahvildar-Zadeh [STZ97] which established a result similar to Theorem 2 in this context, the articles of Struwe [Str02, Str03], and the recent article of Sterbenz and Tataru [ST10] for the general case of solutions without any special invariant properties. We also refer to the works of Rodnianski and Sterbenz [RS], Krieger, Schlag and Tataru [KST08] and Raphaël and Rodnianski $[\mathrm{RR}]$ for the construction of blow-up solutions.

Remark 1.4. Universality of blow-up profiles for a critical equation, as $t$ goes to the blow-up time $T_{+}$(without restriction to a sequence of times), was established, also under a smallness condition, in two cases:

- for the critical $\mathrm{KdV}$ equation

$$
u_{t}=\left(u_{x x}+u^{5}\right)_{x}, \quad x \in \mathbb{R},
$$

by classification of compact solutions of the GKdV equation: see Martel and Merle [MM00, MM01, MM02];

- for the mass-critical NLS equation

$$
i u_{t}=\Delta u+|u|^{4 / N} u, \quad 1 \leq N \leq 5,
$$

by Merle and Raphaël, by classification of solutions that are nondispersive (in a weak sense) [MR04].

See also the subcritical wave equation in dimension one where all blow-up profiles were found by Merle and Zaag [MZ07, MZ08] for general data (see the work of Caffarelli and Friedman for specific data [CF86]).

We next sketch the proof of Theorem 1

Consider a radial, blow-up solution $u$ of 1.1 in space dimension $N=3$ that satisfies (1.7) and assume for simplicity that the blow-up time $T_{+}(u)$ is 1 . As is shown in Section 3 . 
$u$ may be decomposed as the sum of a solution to 1.1 which is well-defined around the blow-up time, and a singular part $a(t, x)$ which is supported in the light cone $|x| \leq$ $1-t$. Choose a sequence $t_{n} \rightarrow 1^{-}$and a Bahouri-Gérard [BG99] profile decomposition associated to the sequence $\left\{\left(a\left(t_{n}\right), \partial_{t} a\left(t_{n}\right)\right)_{n}\right\}$. According to the result of [KM08], the bound (1.7) implies that there is one large profile and that the other profiles are small (see Remark 3.10]. This contrasts with [KM08] where the minimality of the solution imposes automatically that there is only one profile (the large one). In our case, we must show by another mechanism that the small profiles do not exist, which would imply by Theorem 2 that the large profile is $W$, yielding Theorem 1 .

The main idea to exclude the small profiles is that any small block of energy norm decoupled from the main profile would yield, for each time $t_{n}$, a nonnegligible amount of energy norm localized on a light cone ${ }^{1}$ By finite speed of propagation one can show that these small energy blocks, localized in disjoint light cones, sum up, implying the blow-up of the energy norm, which contradicts the bound (1.7). A similar phenomenon is highlighted in the context of the nonlinear Schrödinger equation in [MR05, MR08]. Unfortunately, this strategy can be implemented only for a class of profiles that are very small and exterior in a certain sense (see Proposition 4.4 ), and we must exclude the other small profiles by indirect means (see Sections 5, 7 and 8).

By Proposition 5.1, the existence of a sequence $\tau_{n} \rightarrow 1^{-}$such that $a\left(\tau_{n}\right)$ concentrates at a speed faster than self-similar implies, at least for another sequence of times $t_{n} \rightarrow 1^{-}$, that all profiles are equal to the stationary solution $W$. This property follows from rigidity arguments involving virial type identities. In particular, there cannot be small profiles, and the bound (1.7) implies that $W$ is the only profile for this particular sequence $t_{n}$. This yields the strong condition that the energy of the singular part $E\left(a, \partial_{t} a\right)$ tends to $E(W, 0)$ as $t \rightarrow 1^{-}$(see Corollary 8.3 , which can be combined with the results of [KM08] to complete the proof (see $\$ 8.3$ and $\$ 8.4$ ).

It remains to exclude the case of self-similar concentration, which is the object of Proposition 8.2. Towards a contradiction, we show (as a consequence of the nonexistence of small exterior profiles) that this self-similar concentration, if it exists, must concern the large profile. The solution of (1.1) corresponding to this profile is globally defined and nonscattering backward in time, satisfies a global bound similar to (1.7) for negative times, and is partially located around the light cone $|t|=|x|$ as $t \rightarrow-\infty$. This type of solution is excluded by Proposition 7.1, using the nonexistence, shown in [KM08], of self-similar blow-up solutions of 1.1 which are compact up to scaling.

The outline of the paper is as follows.

After some preliminaries (Section 2), we give in Section 3 general results on type II blow-up solutions of (1.1) in space dimensions $N=3,4,5$. In the next two sections we restrict ourselves to radial solutions in space dimension 3. In Section 4 we show the nonexistence of small exterior profiles for a radial type II blow-up solution. In Section 5 . we assume that the solution does not concentrate at a self-similar rate, and show that

1 This follows from a property of the radial three-dimensional linear wave equation that does not hold in the nonradial setting or in higher dimensions (see Lemma 4.2), which is the main reason why we restricted ourselves to radial solutions in space dimension $N=3$. 
in this case, there exists a sequence $t_{n} \rightarrow T_{+}$such that $u\left(t_{n}\right)$ decomposes as a sum of rescaled stationary solutions.

In the following two sections, we consider solutions of $1.1 p$ that do not blow up in finite time. Section 6 is devoted to the proof of Theorem 2, which is a consequence of the classification result of [DM08]. Section77is concerned with the localization of the energy for globally defined, bounded, nonscattering solutions of 1.1.

Section 8 gathers the results of all previous sections to prove Theorem 1 In Appendix $\mathrm{A}$ we prove some technical properties of profile decompositions. Appendix B shows a simple result on a family of sequences of positive numbers which is needed in some parts of the proof.

In all the article, for sequences of positive numbers $\left\{\alpha_{n}\right\}_{n}$ and $\left\{\beta_{n}\right\}_{n}$, we will write $\alpha_{n} \ll \beta_{n}$ when $\alpha_{n} / \beta_{n} \rightarrow 0$ as $n \rightarrow \infty$, and $\alpha_{n} \approx \beta_{n}$ when $C^{-1} \alpha_{n} \leq \beta_{n} \leq C \alpha_{n}$ for some large constant $n$. We will denote by $o_{n}(1)$ a sequence that goes to 0 as $n$ goes to $\infty$.

Let us mention that the nonradial case will be the object of a subsequent paper to appear in JEMS [DKM].

\section{Preliminaries}

\subsection{Cauchy problem}

The Cauchy problem for equation 1.1] was developed in [Pec84, GSV92, LS95, SS94, SS98, Sog95, Kap94]. If $I$ is an interval, we define

$$
S(I)=L^{\frac{2(N+1)}{N-2}}\left(I \times \mathbb{R}^{N}\right), \quad W(I)=L^{\frac{2(N+1)}{N-1}}\left(I \times \mathbb{R}^{N}\right), \quad N(I)=L^{\frac{2(N+1)}{N+3}}\left(I \times \mathbb{R}^{N}\right) .
$$

Let $\mathrm{S}_{\mathrm{L}}(t)$ be the one-parameter group associated to the linear wave equation. By definition, if $\left(v_{0}, v_{1}\right) \in \dot{H}^{1} \times L^{2}$ and $t \in \mathbb{R}, v(t)=\mathrm{S}_{\mathrm{L}}(t)\left(v_{0}, v_{1}\right)$ is the solution of

$$
\begin{gathered}
\partial_{t}^{2} v-\Delta v=0, \\
v_{\mid t=0}=v_{0}, \quad \partial_{t} v_{\mid t=0}=v_{1} .
\end{gathered}
$$

We have

$$
\mathrm{S}_{\mathrm{L}}(t)\left(v_{0}, v_{1}\right)=\cos (t \sqrt{-\Delta}) v_{0}+\frac{1}{\sqrt{-\Delta}} \sin (t \sqrt{-\Delta}) v_{1} .
$$

By the Strichartz and Sobolev estimates,

$$
\|v\|_{S(\mathbb{R})}+\left\|D_{x}^{1 / 2} v\right\|_{W(\mathbb{R})} \leq C_{S}\left(\left\|v_{0}\right\|_{\dot{H}^{1}}+\left\|v_{1}\right\|_{L^{2}}\right) .
$$

A solution of (1.1) on an interval $I$, where $0 \in I$, is a function $u \in C^{0}\left(I, \dot{H}^{1}\right)$ such that $\partial_{t} u \in C^{0}\left(I, L^{2}\right)$,

$$
J \Subset I \Rightarrow\left\|D_{x}^{1 / 2} u\right\|_{W(J)}+\|u\|_{S(J)}<\infty
$$

satisfying the Duhamel formulation

$$
u(t)=\mathrm{S}(t) u_{0}+\int_{0}^{t} \frac{\sin ((t-s) \sqrt{-\Delta})}{\sqrt{-\Delta}}|u(s)|^{\frac{4}{N-2}} u(s) d s .
$$


We recall there exists a small $\delta_{0}>0$ such that for any interval $I$ containing 0 and any $\left(u_{0}, u_{1}\right) \in \dot{H}^{1} \times L^{2}$ such that

$$
\left\|\mathrm{S}_{\mathrm{L}}(t)\left(u_{0}, u_{1}\right)\right\|_{S(I)}<\delta_{0},
$$

there exists a unique solution $u$ of (1.1) on $I$. Furthermore if $\delta_{0}$ is chosen small enough, this solution satisfies

$$
\|u\|_{S(I))} \leq 2\left\|\mathrm{~S}_{\mathrm{L}}(t)\left(u_{0}, u_{1}\right)\right\|_{S(I)} .
$$

Sticking together these local solutions, we see that for any initial condition $\left(u_{0}, u_{1}\right)$ in the energy space, there exists a unique solution $u$ of $[1.1]$, which is defined on a maximal interval of definition

$$
I_{\max }=I_{\max }\left(u_{0}, u_{1}\right)=\left(T_{-}\left(u_{0}, u_{1}\right), T_{+}\left(u_{0}, u_{1}\right)\right) .
$$

We will often write $I_{\max }(u), T_{ \pm}(u)$, instead of $I_{\max }\left(u_{0}, u_{1}\right), T_{ \pm}\left(u_{0}, u_{1}\right)$.

If $\left\|\mathrm{S}_{\mathrm{L}}(t)\left(u_{0}, u_{1}\right)\right\|_{S(I)}=\delta<\delta_{0}$, then $u$ is close to the linear solution with initial condition $\left(u_{0}, u_{1}\right)$ in the following sense: if $A=\left\|D_{x}^{1 / 2} \mathrm{~S}_{\mathrm{L}}(t)\left(u_{0}, u_{1}\right)\right\|_{W(I)}$, we have

$\left\|u(\cdot)-\mathrm{S}_{\mathrm{L}}(\cdot)\left(u_{0}, u_{1}\right)\right\|_{S(I)}+\sup _{t \in I}\left[\left\|u(t)-\mathrm{S}_{\mathrm{L}}(t)\left(u_{0}, u_{1}\right)\right\|_{\dot{H}^{1}}+\left\|\partial_{t} u(t)-\partial_{t}\left(\mathrm{~S}_{\mathrm{L}}(t)\left(u_{0}, u_{1}\right)\right)\right\|_{L^{2}}\right]$

$$
\leq \operatorname{CA} \frac{4}{N-2}
$$

(see for example [KM06, proof of Theorem 2.7]).

Any solution $u$ of (1.1) satisfies the blow-up criterion 1.2), and the analogue for negative time. As a consequence, if $\|u\|_{S\left(0, T_{+}\right)}<\infty$, then $T_{+}=\infty$. Furthermore in this case, the solution scatters forward in time in $\dot{H}^{1} \times L^{2}$ : there exists a solution $v$ of the linear equation (2.1) such that

$$
\lim _{t \rightarrow \infty}\left[\|u(t)-v(t)\|_{L^{2}}+\left\|\partial_{t} u(t)-\partial_{t} v(t)\right\|_{L^{2}}\right]=0 .
$$

Of course an analogous statement holds backward in time also.

We next recall a long-time perturbation theory result for (1.1) (see Theorem 2.20 of [KM08]).

Theorem 2.1. Let $M>0$. There exists $\varepsilon_{0}=\varepsilon_{0}(M)$ with the following property. Let $I \subset \mathbb{R}$ be a time interval such that $0 \in I$, and $\tilde{u}$ be defined on $I \times \mathbb{R}^{N}$ such that

$$
\|\tilde{u}\|_{S(I)}+\sup _{t \in I}\left[\|\tilde{u}(t)\|_{\dot{H}^{1}}+\left\|\partial_{t} \tilde{u}(t)\right\|_{L^{2}}\right] \leq M, \quad J \Subset I \Rightarrow\left\|D_{x}^{1 / 2} \tilde{u}\right\|_{W(J)}<\infty .
$$

Denote $\left(\tilde{u}_{0}, \tilde{u}_{1}\right)=\left(\tilde{u}(0), \partial_{t} \tilde{u}(0)\right)$. Let $\left(u_{0}, u_{1}\right) \in \dot{H}^{1} \times L^{2}$ and $\varepsilon \in\left(0, \varepsilon_{0}\right)$. Assume

$$
\begin{gathered}
\partial_{t}^{2} \tilde{u}-\Delta \tilde{u}-|\tilde{u}|^{\frac{4}{N-2}} \tilde{u}=e, \quad(t, x) \in I \times \mathbb{R}^{N}, \\
\left\|u_{0}-\tilde{u}_{0}\right\|_{\dot{H}^{1}}+\left\|u_{1}-\tilde{u}_{1}\right\|_{L^{2}}+\left\|D_{x}^{1 / 2} e\right\|_{N(I)} \leq \varepsilon .
\end{gathered}
$$

Then the solution $u$ of (1.1) with initial condition $\left(u_{0}, u_{1}\right)$ satisfies $I_{\max }(u) \subset I$ and for $a \beta_{0}>0$,

$$
\|u\|_{S(I)} \leq C(M), \quad \sup _{t \in I}\left[\|u(t)-\tilde{u}(t)\|_{\dot{H}^{1}}+\left\|\partial_{t} u(t)-\partial_{t} \tilde{u}(t)\right\|_{L^{2}}\right] \leq C(M) \varepsilon^{\beta_{0}} .
$$




\subsection{Remarks on stationary solutions of (1.1)}

Recall from (1.4) the definition of the stationary solution $W$. It is known from the works of T. Aubin [Aub76] and G. Talenti [Tal76] that $W$ is the unique minimizer, up to translation, scaling and multiplication by a scalar constant, for the Sobolev inequality on $\mathbb{R}^{N}$,

$$
\|f\|_{L^{2 N /(N-2)}} \leq C_{N}\|\nabla f\|_{L^{2}} .
$$

By a classical ODE argument, we also have the following uniqueness result:

Claim 2.2. Let $U$ be a (real) $\dot{H}^{1}\left(\mathbb{R}^{N}\right)$ radial solution of

$$
\Delta U+|U|^{\frac{4}{N-2}} U=0
$$

Then

$$
U=0 \quad \text { or } \exists \lambda_{0}>0, U= \pm \frac{1}{\lambda_{0}^{(N-2) / 2}} W\left(\frac{x}{\lambda_{0}}\right) .
$$

Note that the equation $\Delta W+W^{(N+2) /(N-2)}=0$ implies $E(W, 0)=N^{-1} \int|\nabla W|^{2}>0$. This fact is used to prove the following variational properties of $W$ which will be needed throughout the paper.

Claim 2.3. Let $v \in \dot{H}^{1}$. Then

$$
\begin{aligned}
\|\nabla v\|_{L^{2}}^{2} \leq\|\nabla W\|_{L^{2}}^{2} \text { and } E(v, 0) & \leq E(W, 0) \\
\Rightarrow\|\nabla v\|_{L^{2}}^{2} & \leq \frac{\|\nabla W\|_{L^{2}}^{2}}{E(W, 0)} E(v, 0)=N E(v, 0) .
\end{aligned}
$$

Furthermore, if $\|\nabla v\|_{L^{2}}^{2} \leq\left(\frac{N}{N-2}\right)^{(N-2) / 2}\|\nabla W\|_{L^{2}}^{2}$, then $E(v, 0) \geq 0$.

Proof. The first part of the claim is shown in [DM08 proof of Claim 2.4]. For the second part, write

$E(v, 0)=\frac{1}{2} \int|\nabla v|^{2}-\frac{N-2}{2 N} \int|v|^{\frac{2 N}{N-2}} \geq \frac{1}{2} \int|\nabla v|^{2}-\frac{N-2}{2 N} C_{N}^{\frac{2 N}{N-2}}\left(\int|\nabla v|^{2}\right)^{\frac{N}{N-2}}$,

where $C_{N}$ is the best constant in the Sobolev inequality $\|v\|_{2 N /(N-2)} \leq C_{N}\|\nabla v\|_{L^{2}}$. Let $y=\int|\nabla v|^{2}$ and assume that $E(v, 0)$ is negative. Then

$$
0>\frac{1}{2} y-\frac{N-2}{2 N} C_{N}^{\frac{2 N}{N-2}} y^{\frac{N}{N-2}} .
$$

This shows that $y \geq y_{*}$, where $y_{*}$ is the unique positive solution of $\frac{1}{2} y-\frac{N-2}{2 N} C_{N}^{\frac{2 N}{N-2}} y^{\frac{N}{N-2}}$ $=0$. Using that $C_{N}^{-N}=\int|\nabla W|^{2}$, we obtain $y_{*}=\left(\frac{N}{N-2}\right)^{(N-2) / 2} \int|\nabla W|^{2}$, which concludes the proof. 


\subsection{Profile decomposition}

We recall here the profile decomposition of H. Bahouri and P. Gérard [BG99]. This paper is written in space dimension $N=3$ but the results stated below hold in all dimensions $N \geq 3$. See also [BC85] and [Lio85] for the elliptic case and [MV98] for the Schrödinger equation.

Choose a sequence $\left\{\left(v_{0, n}, v_{1, n}\right)\right\}_{n}$ which is bounded in $\dot{H}^{1} \times L^{2}$. Let $\left\{U_{\mathrm{L}}^{j}\right\}_{j \geq 0}$ be a sequence of solutions of the linear equation 2.1], with initial data $\left(U_{0}^{j}, U_{1}^{j}\right) \in \dot{H}^{1} \times L^{2}$, and $\left(\lambda_{j, n}, x_{j, n}, t_{j, n}\right) \in(0, \infty) \times \mathbb{R}^{N} \times \mathbb{R}, j, n \in \mathbb{N}$, be a family of parameters satisfying the pseudo-orthogonality relation

$$
j \neq k \Rightarrow \lim _{n \rightarrow \infty}\left[\frac{\lambda_{j, n}}{\lambda_{k, n}}+\frac{\lambda_{k, n}}{\lambda_{j, n}}+\frac{\left|t_{j, n}-t_{k, n}\right|}{\lambda_{j, n}}+\frac{\left|x_{j, n}-x_{k, n}\right|}{\lambda_{j, n}}\right]=\infty .
$$

We say that $\left\{\left(v_{0, n}, v_{1, n}\right)\right\}_{n}$ admits a profile decomposition $\left\{U_{\mathrm{L}}^{j}\right\}_{j},\left\{\lambda_{j, n}, x_{j, n}, t_{j, n}\right\}_{j, n}$ when

$$
\left\{\begin{array}{l}
v_{0, n}=\sum_{j=1}^{J} \frac{1}{\lambda_{j, n}^{N-2) / 2}} U_{\mathrm{L}}^{j}\left(\frac{-t_{j, n}}{\lambda_{j, n}}, \frac{x-x_{j, n}}{\lambda_{j, n}}\right)+w_{0, n}^{J}(x), \\
v_{1, n}=\sum_{j=1}^{J} \frac{1}{\lambda_{j, n}^{N / 2}} \partial_{t} U_{\mathrm{L}}^{j}\left(\frac{-t_{j, n}}{\lambda_{j, n}}, \frac{x-x_{j, n}}{\lambda_{j, n}}\right)+w_{1, n}^{J}(x),
\end{array}\right.
$$

with

$$
\lim _{n \rightarrow \infty} \limsup _{J \rightarrow \infty}\left\|w_{n}^{J}\right\|_{S(\mathbb{R})}=0,
$$

where $w_{n}^{J}$ is the solution of 2.1) with initial conditions $\left(w_{0, n}^{J}, w_{1, n}^{J}\right)$. Then:

Theorem 2.4 ([तG99]). If the sequence $\left\{\left(v_{0, n}, v_{1, n}\right)\right\}_{n}$ is bounded in the energy space $\dot{H}^{1} \times L^{2}$, there always exists a subsequence of $\left\{\left(v_{0, n}, v_{1, n}\right)\right\}_{n}$ which admits a profile decomposition. Furthermore,

$$
j \leq J \Rightarrow\left(\lambda_{j, n}^{(N-2) / 2} w_{n}^{J}\left(t_{j, n}, x_{j, n}+\lambda_{j, n} y\right), \lambda_{j, n}^{N / 2} \partial_{t} w_{n}^{J}\left(t_{j, n}, x_{j, n}+\lambda_{j, n} y\right)\right) \underset{n \rightarrow \infty}{\longrightarrow} 0,
$$

weakly in $\dot{H}_{y}^{1} \times L_{y}^{2}$, and the following Pythagorean expansions hold for all $J \geq 1$ :

$$
\begin{gathered}
\left\|v_{0, n}\right\|_{\dot{H}^{1}}^{2}=\sum_{j=1}^{J}\left\|U_{\mathrm{L}}^{j}\left(\frac{-t_{j, n}}{\lambda_{j, n}}\right)\right\|_{\dot{H}^{1}}^{2}+\left\|w_{0, n}^{J}\right\|_{\dot{H}^{1}}^{2}+o_{n}(1) \\
\left\|v_{1, n}\right\|_{L^{2}}^{2}=\sum_{j=1}^{J}\left\|\partial_{t} U_{1}^{j}\left(\frac{-t_{j, n}}{\lambda_{j, n}}\right)\right\|_{L^{2}}^{2}+\left\|w_{1, n}^{J}\right\|_{L^{2}}^{2}+o_{n}(1) \\
E\left(v_{0, n}, v_{1, n}\right)=\sum_{j=1}^{J} E\left(U_{\mathrm{L}}^{j}\left(-\frac{t_{j, n}}{\lambda_{j, n}}\right), \partial_{t} U_{\mathrm{L}}^{j}\left(-\frac{t_{j, n}}{\lambda_{j, n}}\right)\right)+E\left(w_{0, n}^{J}, w_{1, n}^{J}\right)+o_{n}(1)
\end{gathered}
$$


Replacing $U_{\mathrm{L}}^{j}(t, x)$ by $V_{\mathrm{L}}^{j}(t, x)=\frac{1}{\lambda_{j}^{(N-2) / 2}} U_{\mathrm{L}}^{j}\left(\frac{t-t_{j}}{\lambda_{j}}, \frac{x-x_{j}}{\lambda_{j}}\right)$ for some good choice of the parameters $\lambda_{j}, t_{j}, x_{j}$, and extracting subsequences, we can always assume that one of the following two cases occurs:

$$
\forall n, t_{j, n}=0 \quad \text { or } \quad \lim _{n \rightarrow \infty} \frac{t_{j, n}}{\lambda_{j, n}} \in\{-\infty, \infty\}
$$

We will need the following bound on the parameters:

Lemma 2.5. Let $v_{n}$ be as above and $\left\{\mu_{n}\right\}_{n}$ be a sequence of positive numbers. Assume

$$
\lim _{R \rightarrow \infty} \limsup _{n \rightarrow \infty} \int_{|x| \geq R \mu_{n}}\left[\left|\nabla v_{0, n}\right|^{2}+v_{1, n}^{2}\right] d x=0 .
$$

Then for all $j$, the sequences $\left\{\lambda_{j, n} / \mu_{n}\right\}_{n},\left\{t_{j, n} / \mu_{n}\right\}_{n}$ and $\left\{x_{j, n} / \mu_{n}\right\}_{n}$ are bounded. Furthermore, there is at most one $j$ such that $\left\{\lambda_{j, n} / \mu_{n}\right\}_{n}$ does not converge to 0 .

Proof. The case $\mu_{n}=1$ follows from [BG99, pp. 154-155]. For the general case, apply the result of the case $\mu_{n}=1$ to the rescaled sequence $\left\{\left(\tilde{v}_{0, n}, \tilde{v}_{1, n}\right)\right\}_{n}$ defined by

$$
\tilde{v}_{0, n}(t, x)=\mu_{n}^{N / 2-1} v_{0, n}\left(\mu_{n} x\right), \quad \tilde{v}_{1, n}(t, x)=\mu_{n}^{N / 2} v_{1, n}\left(\mu_{n} x\right) .
$$

Notation 2.6. For any profile decomposition with profiles $\left\{U_{\mathrm{L}}^{j}\right\}$ and parameters $\left\{\lambda_{j, n}, t_{j, n}, x_{j, n}\right\}$, we will denote by $\left\{U^{j}\right\}$ the nonlinear profiles associated with $\left\{U_{\mathrm{L}}^{j}\left(-t_{j, n} / \lambda_{j, n}\right), \partial_{t} U_{\mathrm{L}}^{j}\left(-t_{j, n} / \lambda_{j, n}\right)\right\}$, which are the unique solutions of 1.1 such that for all $n,-t_{j, n} / \lambda_{j, n} \in I_{\max }\left(U^{j}\right)$ and

$$
\lim _{n \rightarrow \infty}\left[\left\|U^{j}\left(\frac{-t_{j, n}}{\lambda_{j, n}}\right)-U_{\mathrm{L}}^{j}\left(\frac{-t_{j, n}}{\lambda_{j, n}}\right)\right\|_{\dot{H}^{1}}+\left\|\partial_{t} U^{j}\left(\frac{-t_{j, n}}{\lambda_{j, n}}\right)-\partial_{t} U_{\mathrm{L}}^{j}\left(\frac{-t_{j, n}}{\lambda_{j, n}}\right)\right\|_{L^{2}}\right]=0 .
$$

Assuming 2.17), the proof of the existence of $U^{j}$ follows from the local existence for (1.1) if $t_{j, n}=0$ and from the existence of wave operators for equation (1.1) if $t_{j, n} / \lambda_{j, n}$ tends to $\pm \infty$. By the Strichartz inequalities for the linear problem, and the small data Cauchy theory (see 2.7)), if $\lim _{n \rightarrow \infty}-t_{j, n} / \lambda_{j, n}=\infty$, then $T_{+}\left(U^{j}\right)=\infty$ and

$$
s_{0}>T_{-}\left(U^{j}\right) \Rightarrow\left\|U^{j}\right\|_{S\left(s_{0}, \infty\right)}<\infty ;
$$

an analogous statement holds in the case $\lim _{n \rightarrow \infty} t_{j, n} / \lambda_{j, n}=\infty$.

Notation 2.7. We will often write, for the sake of simplicity,

$$
\begin{aligned}
U_{n}^{j}(t, x) & =\frac{1}{\lambda_{j, n}^{N / 2-1}} U^{j}\left(\frac{t-t_{j, n}}{\lambda_{j, n}}, \frac{x-x_{j, n}}{\lambda_{j, n}}\right), \\
U_{\mathrm{L}, n}^{j}(t, x) & =\frac{1}{\lambda_{j, n}^{N / 2-1}} U_{\mathrm{L}}^{j}\left(\frac{t-t_{j, n}}{\lambda_{j, n}}, \frac{x-x_{j, n}}{\lambda_{j, n}}\right) .
\end{aligned}
$$


We will need the following approximation result, which follows from Theorem 2.1 and is an adaptation to the focusing case of the result of Bahouri-Gérard (see the Main Theorem, p. 135 in [BG99]).

Proposition 2.8. Let $\left\{\left(v_{0, n}, v_{1, n}\right)\right\}_{n}$ be a bounded sequence in $\dot{H}^{1} \times L^{2}$ which admits the profile decomposition (2.11). Let $\theta_{n} \in(0, \infty)$. Assume that for all $j, n$,

$$
\frac{\theta_{n}-t_{j, n}}{\lambda_{j, n}}<T_{+}\left(U^{j}\right) \quad \text { and } \quad \limsup _{n \rightarrow \infty}\left\|U^{j}\right\|_{S\left(-t_{j, n} / \lambda_{j, n},\left(\theta_{n}-t_{j, n}\right) / \lambda_{j, n}\right)}<\infty
$$

Let $u_{n}$ be the solution of (1.1) with initial data $\left(v_{0, n}, v_{1, n}\right)$. Then for large $n, u_{n}$ is defined on $\left[0, \theta_{n}\right)$,

$$
\limsup _{n \rightarrow \infty}\left\|u_{n}\right\|_{S\left(0, \theta_{n}\right)}<\infty
$$

and

$$
\forall t \in\left[0, \theta_{n}\right), \quad u_{n}(t, x)=\sum_{j=1}^{J} U_{n}^{j}(t, x)+w_{n}^{J}(t, x)+r_{n}^{J}(t, x)
$$

where

$$
\lim _{n \rightarrow \infty} \limsup _{J \rightarrow \infty}\left[\left\|r_{n}^{J}\right\| S\left(0, \theta_{n}\right)+\sup _{t \in\left(0, \theta_{n}\right)}\left(\left\|\nabla r_{n}^{J}(t)\right\|_{L^{2}}+\left\|\partial_{t} r_{n}^{J}(t)\right\|_{L^{2}}\right)\right]=0
$$

An analogous statement holds if $\theta_{n}<0$.

Remark 2.9. Assume that for all $j$, at least one of the following occurs:

(a) $\left\|U_{0}^{j}\right\|_{\dot{H}^{1}}+\left\|U_{1}^{j}\right\|_{L^{2}}<\delta_{0} / C_{S}$, where the constant $C_{S}$ is given by the Strichartz estimate 2.3 and $\delta_{0}$ by the small data theory;

(b) $\lim _{n \rightarrow \infty} \frac{-t_{j, n}}{\lambda_{j, n}}=\infty$,

(c) $\limsup _{n \rightarrow \infty} \frac{\theta_{n}-t_{j, n}}{\lambda_{j, n}}<T_{+}\left(U^{j}\right)$.

Then (2.8) holds. Indeed in case (a), this follows from (2.3) and the small data theory. In case (b), it follows directly from the small data theory: see 2.19). It remains to treat case (c), when $t_{j, n}=0$ or $-t_{j, n} / \lambda_{j, n} \rightarrow-\infty$. If $t_{j, n}=0$, then by definition $T_{-}\left(U^{j}\right)<0$ and 2.8) is a consequence of (2.4) and (c). If $-t_{j, n} / \lambda_{j, n} \rightarrow-\infty$, then the analogue of 2.19) for negative times and (c) imply 2.8.

Remark 2.10. When $N$ is odd, under the assumptions of Proposition 2.8, we have localized pseudo-orthogonality properties for all time of the interval $\left(0, \theta_{n}\right)$ as follows: let $\tau_{n} \in\left(0, \theta_{n}\right)$ for all $n,\left\{\mu_{n}\right\}$ be any sequence of positive numbers, and $\chi \in C_{0}^{\infty}\left(\mathbb{R}^{N}\right)$ be 
radial and such that $\chi=1$ in a neighborhood of 0 . Then, if $\varphi=1, \varphi=\chi$ or $\varphi=1-\chi$, one can show after extraction the following Pythagorean expansion:

$$
\begin{aligned}
& \int \varphi\left(\frac{x}{\mu_{n}}\right)\left|\nabla_{t, x} u\left(\tau_{n}\right)\right|^{2} d x \\
& \quad=\sum_{j=1}^{J} \int \varphi\left(\frac{x}{\mu_{n}}\right)\left|\nabla_{t, x} U_{n}^{j}\left(\tau_{n}\right)\right|^{2} d x+\int \varphi\left(\frac{x}{\mu_{n}}\right)\left|\nabla_{t, x} w_{n}^{J}\left(\tau_{n}\right)\right|^{2} d x+o_{n}(1),
\end{aligned}
$$

where $\left|\nabla_{t, x} u\right|^{2}=\left(\partial_{t} u\right)^{2}+\left|\nabla_{x} u\right|^{2}$. This follows easily from Claim A.1 in the appendix and we omit the proof.

Sketch of proof of Proposition 2.8. Denote the nonlinearity by $F(u)=|u|^{4 /(N-2)} u$. Let

$$
\tilde{u}_{n}^{J}(t, x)=\sum_{j=1}^{J} \frac{1}{\lambda_{j, n}^{(N-2) / 2}} U^{j}\left(\frac{t-t_{j, n}}{\lambda_{j, n}}, \frac{x-x_{j, n}}{\lambda_{j, n}}\right)+w_{n}^{J}(t, x) .
$$

We will apply Theorem 2.1 to $\tilde{u}_{n}$ and $u_{n}$ for large $n$.

We notice that there exists $J_{0}>0$ such that

$$
\forall j \geq J_{0}+1, \quad\left\|U_{\mathrm{L}}^{j}\right\|_{S(\mathbb{R})}<\delta_{0},
$$

where $\delta_{0}$ is given by the small data theory for 1.1 . Indeed, this is an immediate consequence of the Pythagorean expansions 2.14, 2.15) and Strichartz estimates. Thus we can use the small data theory which implies, by (2.7),

$$
\forall j \geq J_{0}+1, \quad\left\|U^{j}\right\|_{S(\mathbb{R})} \leq C\left(\left\|U_{0}^{j}\right\|_{\dot{H}^{1}}+\left\|U_{1}^{j}\right\|_{L^{2}}\right) .
$$

Fixing a large $J$, one can show, as a consequence of the orthogonality 2.10 of the parameters,

$$
\begin{aligned}
& \left\|\sum_{j=1}^{J} \frac{1}{\lambda_{j, n}^{(N-2) / 2}} U^{j}\left(\frac{t-t_{j, n}}{\lambda_{j, n}}, \frac{x-x_{j, n}}{\lambda_{j, n}}\right)\right\|_{S\left(0, \theta_{n}\right)}^{\frac{2(N+1)}{N-2}} \\
& \quad=\sum_{j=1}^{J}\left\|\frac{1}{\lambda_{j, n}^{(N-2) / 2}} U^{j}\left(\frac{t-t_{j, n}}{\lambda_{j, n}}, \frac{x-x_{j, n}}{\lambda_{j, n}}\right)\right\|_{S\left(0, \theta_{n}\right)}^{\frac{2(N+1)}{N-2}}+o_{n}(1) .
\end{aligned}
$$

Combining this with 2.25) and the Pythagorean expansions 2.14, 2.15, we get

$$
\limsup _{J \rightarrow \infty} \limsup _{n \rightarrow \infty}\left\|\tilde{u}_{n}^{J}\right\|_{S\left(0, \theta_{n}\right)}<\infty .
$$

Let $J \geq J_{0}$ and $e_{n}^{J}=\left(\partial_{t}^{2}-\Delta\right) \tilde{u}_{n}^{J}-F\left(\tilde{u}_{n}^{J}\right)$. Then

$$
e_{n}^{J}(t, x)=\sum_{j=1}^{J} F\left(\frac{1}{\lambda_{j, n}^{(N-2) / 2}} U^{j}\left(\frac{t-t_{j, n}}{\lambda_{j, n}}\right)\right)-F\left(\sum_{j=1}^{J} \frac{1}{\lambda_{j, n}^{(N-2) / 2}} U^{j}\left(\frac{t-t_{j, n}}{\lambda_{j, n}}\right)+w_{n}^{J}\right) .
$$


From 2.8 and again the orthogonality 2.10 of the parameters, we can deduce

$$
\lim _{J \rightarrow \infty} \limsup _{n \rightarrow \infty}\left\|D_{x}^{1 / 2} e_{n}^{J}\right\|_{N\left(0, \tau_{n}\right)}=0
$$

Furthermore

$$
\tilde{u}_{n}^{J}(0)=u_{n}^{J}(0), \quad \partial_{t} \tilde{u}_{n}^{J}(0)=\partial_{t} u_{n}^{J}(0),
$$

which yields by Theorem 2.1 the conclusion of the proposition.

We will also need the following technical claim. The proof is postponed to Appendix A

Claim 2.11. Assume that $N$ is odd. Let $\left\{w_{n}\right\}$ be a sequence of radial solutions to the linear wave equation 2.1] with bounded energy and such that

$$
\lim _{n \rightarrow \infty}\left\|w_{n}\right\|_{S(\mathbb{R})}=0 .
$$

Let $\left(w_{0, n}, w_{1, n}\right)$ be the initial data of $w_{n}, \chi \in C_{0}^{\infty}\left(\mathbb{R}^{N}\right)$ be radial and such that $\chi=1$ around the origin, and $\left\{\tilde{\lambda}_{n}\right\}$ be a sequence of positive numbers. Consider the solution $\tilde{w}_{n}$ to 2.1 with initial data $\left(\tilde{w}_{0, n}, \tilde{w}_{1, n}\right)=\left(\varphi\left(|x| / \tilde{\lambda}_{n}\right) w_{0, n}, \varphi\left(|x| / \tilde{\lambda}_{n}\right) w_{1, n}\right)$, where $\varphi=\chi$ or $\varphi=1-\chi$. Then

$$
\lim _{n \rightarrow \infty}\left\|\tilde{w}_{n}\right\|_{S(\mathbb{R})}=0
$$

\section{Description of general type II blow-up solutions}

In this section we consider a general type II blow-up solution of (1.1) in space dimension $N \in\{3,4,5\}$, that is, a solution $u$ bounded in the energy space and such that $T_{+}(u)<\infty$. We do not assume that $u$ is spherically symetric.

Definition 3.1. Let $x_{0} \in \mathbb{R}^{N}$. We will say that the point $x_{0}$ is regular if

$$
\forall \varepsilon>0, \exists R, \forall t \in\left[0, T_{+}(u)\right), \quad \int_{\left|x-x_{0}\right| \leq R}\left[|\nabla u|^{2}+\frac{u^{2}}{\left|x-x_{0}\right|^{2}}+\left(\partial_{t} u\right)^{2}\right] \leq \varepsilon .
$$

If $x_{0}$ is not regular, we will say that it is singular. We will denote by $S$ the set of singular points.

Theorem 3.2. Let $u$ be a solution of (1.1) with type II blow-up forward in time, and $T_{+}=$ $T_{+}(u)$ the blow-up time. Then there exists $K \in \mathbb{N}^{*}$ and $K$ distinct points $m_{1}, \ldots, m_{K}$ of $\mathbb{R}^{N}$ such that $S=\left\{m_{1}, \ldots, m_{K}\right\}$. Furthermore there exists $\left(v_{0}, v_{1}\right) \in \dot{H}^{1} \times L^{2}$ such that

$$
\left(u(t), \partial_{t} u(t)\right) \underset{t \rightarrow T_{+}}{\longrightarrow}\left(v_{0}, v_{1}\right) \quad \text { weakly in } \dot{H}^{1} \times L^{2} .
$$

If $\varphi \in C_{0}^{\infty}\left(\mathbb{R}^{N}\right)$ is equal to 1 around each singular point, we have

$$
\lim _{t \rightarrow T_{+}}\left[\left\|(1-\varphi)\left(u(t)-v_{0}\right)\right\|_{\dot{H}^{1}}+\left\|(1-\varphi)\left(\partial_{t} u(t)-v_{1}\right)\right\|_{L^{2}}\right]=0 .
$$


Furthermore, if $k \in\{1, \ldots, K\}$, assuming moreover $N=3,4$ for (3.4),

$$
\begin{aligned}
& \limsup _{t \rightarrow T_{+}} \int_{\left|x-m_{k}\right| \leq\left|t-T_{+}\right|}\left[|\nabla u(t, x)|^{2}+\left|\partial_{t} u(t, x)\right|^{2}\right] \geq \int|\nabla W|^{2}, \\
& \liminf _{t \rightarrow T_{+}} \int_{\left|x-m_{k}\right| \leq\left|t-T_{+}\right|}\left[|\nabla u(t, x)|^{2}+\left|\partial_{t} u(t, x)\right|^{2}\right] \geq \frac{2}{N} \int|\nabla W|^{2} .
\end{aligned}
$$

Definition 3.3. Under the assumptions of Theorem 3.2, le $v$ be the solutions of (1.1) such that $\left(v\left(T_{+}\right), \partial_{t} v\left(T_{+}\right)\right)=\left(v_{0}, v_{1}\right)$. We will call $v$ the regular part of $u$ at the blow-up time $T_{+}$, and $a=u-v$ the singular part of $u$. Note that 3.3 implies, together with the finite speed of propagation, that

$$
\operatorname{supp} a \subset \bigcup_{k=1}^{K}\left\{(t, x):\left|x-m_{k}\right| \leq\left|t-T_{+}\right|\right\} .
$$

This section is divided into two parts. In $\$ 3.1$, we perform a first analysis of the behavior of $u$ around each singular point, showing (3.2) and (3.3). In $\$ 3.2$, we write a profile decomposition of the solution around each singular point to show (3.4) and 3.5).

We will assume throughout without loss of generality that the blow-up time is $T_{+}(u)=1$.

\subsection{Generalities on regular and singular points}

Lemma 3.4. There exists a constant $\delta_{1}>0$ with the following properties:

(a) for all $x_{0} \in \mathbb{R}^{N}, t_{0} \in(0,1)$ and $R>0$, if

$$
\int_{\left|x-x_{0}\right| \leq\left|t_{0}-1\right|+R}\left[\left|\nabla u\left(t_{0}\right)\right|^{2}+\left|\partial_{t} u\left(t_{0}\right)\right|^{2}+\frac{1}{\left|x-x_{0}\right|^{2}}\left|u\left(t_{0}\right)\right|^{2}\right] \leq \delta_{1},
$$

and $\varphi \in C_{0}^{\infty}\left(\mathbb{R}^{N}\right)$ has compact support in $\left\{\left|x-x_{0}\right| \leq R\right\}$, then $\left(\varphi u(t), \varphi \partial_{t} u(t)\right)$ has a limit in $\dot{H}^{1} \times L^{2}$ as $t \stackrel{<}{\rightarrow}$;

(b) for all $t_{0} \in(0,1)$ and $R>0$, if

$$
\int_{|x| \geq R}\left[\left|\nabla u\left(t_{0}\right)\right|^{2}+\left|\partial_{t} u\left(t_{0}\right)\right|^{2}+\frac{1}{|x|^{2}}\left|u\left(t_{0}\right)\right|^{2}\right] \leq \delta_{1},
$$

and $\varphi \in C^{\infty}\left(\mathbb{R}^{N}\right)$ is equal to 1 at infinity and is supported in the set $|x| \geq R+\left|1-t_{0}\right|$, then $\left(\varphi u(t), \varphi \partial_{t} u(t)\right)$ has a limit in $\dot{H}^{1} \times L^{2}$ as $t \stackrel{<}{\rightarrow} 1$.

Proof. Let us prove (a). Assume that for some parameter $\eta_{0}>0$ to be determined later,

$$
\int_{\left|x-x_{0}\right| \leq\left|t_{0}-1\right|+R}\left[\left|\nabla u\left(t_{0}\right)\right|^{2}+\left|\partial_{t} u\left(t_{0}\right)\right|^{2}+\frac{1}{\left|x-x_{0}\right|^{2}}\left|u\left(t_{0}\right)\right|^{2}\right] \leq \eta_{0} E(W, 0) .
$$


If $\eta_{0}$ is chosen small enough, then, by a standard extension theorem, there exist $\tilde{u}_{0} \in \dot{H}^{1}$ and $\tilde{u}_{1} \in L^{2}$ compactly supported on $\mathbb{R}^{N}$ and such that

$$
\begin{aligned}
& \tilde{u}_{0}(x)=u\left(t_{0}, x\right) \quad \text { and } \quad \tilde{u}_{1}=\partial_{t} u\left(t_{0}, x\right) \quad \text { if }\left|x-x_{0}\right| \leq\left|t_{0}-1\right|+R, \\
& \int_{\mathbb{R}^{N}}\left[\left|\nabla \tilde{u}_{0}\right|^{2}+\left|\tilde{u}_{1}\right|^{2}+\frac{1}{\left|x-x_{0}\right|^{2}}\left|\tilde{u}_{0}\right|^{2}\right] \leq C \eta_{0} E(W, 0)<E(W, 0) .
\end{aligned}
$$

Consider the solution $\tilde{u}$ of 1.1 with initial condition $\left(\tilde{u}_{0}, \tilde{u}_{1}\right)$ at $t=t_{0}$. By 3.7 , we have

$$
\begin{aligned}
E\left(\tilde{u}_{0}, \tilde{u}_{1}\right) & =\frac{1}{2} \int_{\mathbb{R}^{N}}\left|\nabla \tilde{u}_{0}\right|^{2}+\frac{1}{2} \int_{\mathbb{R}^{N}}\left|\tilde{u}_{1}\right|^{2}-\frac{N-2}{2 N} \int_{\mathbb{R}^{N}}\left|\tilde{u}_{0}\right|^{\frac{2 N}{N-2}}<E(W, 0), \\
\left\|\nabla \tilde{u}_{0}\right\|_{L^{2}}^{2} & \leq E(W, 0)<\int|\nabla W|^{2} .
\end{aligned}
$$

By the result of Kenig-Merle [KM08], $\tilde{u}$ is globally defined. The mapping $t \mapsto$ $\left(\tilde{u}(t), \partial_{t} \tilde{u}(t)\right)$ is continuous from $\mathbb{R}$ to $\dot{H}^{1} \times L^{2}$. By finite speed of propagation and 3.6 ,

$\forall t \in\left[t_{0}, 1\right], \forall x \in \mathbb{R}^{N},\left|x-x_{0}\right| \leq|t-1|+R \Rightarrow u(t, x)=\tilde{u}(t, x), \partial_{t} u(t, x)=\partial_{t} \tilde{u}(t, x)$.

In particular, $\left(\varphi u(t), \varphi \partial_{t} u(t)\right)=\left(\varphi \tilde{u}(t), \varphi \partial_{t} \tilde{u}(t)\right)$ has a limit as $t \rightarrow 1$, which concludes the proof of case (a).

Case (b) is similar. Indeed, in this case, if $\delta_{1}$ is small enough, there exist $\tilde{u}_{0}$ and $\tilde{u}_{1}$ such that

$$
\begin{gathered}
\tilde{u}_{0}(x)=u\left(t_{0}, x\right) \quad \text { and } \quad \tilde{u}_{1}=\partial_{t} u\left(t_{0}, x\right) \text { if }|x| \geq R, \\
\int_{\mathbb{R}^{N}}\left[\left|\nabla \tilde{u}_{0}\right|^{2}+\left|\tilde{u}_{1}\right|^{2}+\frac{1}{|x|^{2}}\left|\tilde{u}_{0}\right|^{2}\right]<E(W, 0) .
\end{gathered}
$$

Consider the solution $\tilde{u}$ with initial data $\left(\tilde{u}_{0}, \tilde{u}_{1}\right)$ at $t=t_{0}$. By finite speed of propagation, $u$ and $\tilde{u}$ coincide if $|x|>\left|t_{0}-t\right|+R$, and the result follows again by the global existence of $\tilde{u}$.

Corollary 3.5. For any singular point $m$, and all $t \in I_{\max }=I_{\max }(u)$,

$$
\delta_{1} \leq \int_{|x-m| \leq|t-1|}\left[|\nabla u(t)|^{2}+\left|\partial_{t} u(t)\right|^{2}+\frac{1}{|x-m|^{2}}|u(t)|^{2}\right],
$$

where $\delta_{1}$ is given by Lemma 3.4 Furthermore, the set $S$ of singular points is finite.

Proof. The finiteness of $S$ follows immediately from (3.8) and the fact that the blow-up is of type II.

Let us show 3.8). Towards a contradiction, consider a singular point $m$, and assume that for some $t_{0} \in I_{\max }$ and $\varepsilon>0$,

$$
\int_{|x-m| \leq\left|t_{0}-1\right|+\varepsilon}\left[\left|\nabla u\left(t_{0}\right)\right|^{2}+\left|\partial_{t} u\left(t_{0}\right)\right|^{2}+\frac{1}{|x-m|^{2}}\left|u\left(t_{0}\right)\right|^{2}\right]<\delta_{1} .
$$


Let $\varphi \in C_{0}^{\infty}\left(\mathbb{R}^{N}\right)$ be such that $\varphi(x)=0$ if $|x-m| \geq \varepsilon$ and $\varphi(x)=1$ if $|x-m| \leq \varepsilon / 2$. By Lemma 3.4 $\left(\varphi u, \varphi \partial_{t} u\right)$ converges in $\dot{H}^{1} \times L^{2}$ as $t$ tends to 1 , contradicting, in view of the continuous embedding of $\dot{H}^{1}$ into $L^{2}\left(|x-m|^{-2} d x\right)$, the assumption that $m$ is a singular blow-up point.

We have proven that for all $t \in I_{\max }$ and all $\varepsilon>0$,

$$
\delta_{1} \leq \int_{|x-m| \leq|t-1|+\varepsilon}\left[|\nabla u(t)|^{2}+\left|\partial_{t} u(t)\right|^{2}+\frac{1}{|x-m|^{2}}|u(t)|^{2}\right],
$$

concluding the proof of 3.8.

We are now ready to prove 3.2 and 3.3 of Theorem 3.2. Let us first show that $\left(u(t), \partial_{t} u(t)\right)$ has a weak limit in $\dot{H}^{1} \times L^{2}$ as $t \stackrel{<}{\rightarrow} 1$. It is equivalent to show that all weak limits of sequences $\left\{\left(u\left(t_{n}\right), \partial_{t} u\left(t_{n}\right)\right)\right\}_{n}$, where $t_{n} \stackrel{<}{\rightarrow} 1$, coincide. For this, notice that the definition of a regular point and Lemma 3.4 show that if $\left(v_{0}, v_{1}\right)$ and $\left(\tilde{v}_{0}, \tilde{v}_{1}\right)$ are such limits, then they must coincide around any regular point. As the set of singular points is finite, this shows as desired that $\left(v_{0}, v_{1}\right)=\left(\tilde{v}_{0}, \tilde{v}_{1}\right)$. Denote

$$
\left(v_{0}, v_{1}\right)=w-\lim _{t \rightarrow 1}\left(u(t), \partial_{t} u(t)\right)
$$

By Lemma 3.4 a,$\left(u, \partial_{t} u\right)$ has a limit in $\dot{H}_{\text {loc }}^{1}\left(\mathbb{R}^{N} \backslash S\right) \times L_{\text {loc }}^{2}\left(\mathbb{R}^{N} \backslash S\right)$ as $t$ goes to 1 . The uniqueness of limits shows that this limit must be $\left(v_{0}, v_{1}\right)$. Using Lemma 3.4 , we conclude that the convergence to $v$ is also global, hence (3.3).

We finish this part by noting that there is at least one singular point. If not, 3.3 shows that $\left(u(t), \partial_{t} u(t)\right)$ has a limit as $t \rightarrow 1$, which shows that 1 is not the maximal positive time of existence, a contradiction.

\subsection{Bounds from below on the norm of the main profile}

In this subsection we will complete the proof of Theorem 3.2 by studying the behavior of $u$ in the neighborhood of singular points by using a profile decomposition. We assume that

$$
0 \in S
$$

Choose an increasing sequence $\left\{\tau_{n}\right\} \in\left(t_{0}, 1\right)^{\mathbb{N}}$ that tends to 1 and a function $\psi \in$ $C_{0}^{\infty}\left(\mathbb{R}^{N}\right)$ such that $\psi=1$ close to 0 and supp $\psi \cap S=\{0\}$. After extracting a subsequence, we can assume that there exists a profile decomposition

$$
\left\{\begin{array}{l}
\psi u\left(\tau_{n}\right)-\psi v\left(\tau_{n}\right)=\sum_{j=1}^{J} \frac{1}{\lambda_{j, n}^{N / 2-1}} U_{\mathrm{L}}^{j}\left(\frac{-t_{j, n}}{\lambda_{j, n}}, \frac{x-x_{j, n}}{\lambda_{j, n}}\right)+w_{0, n}(x), \\
\psi \frac{\partial u}{\partial t}\left(\tau_{n}\right)-\psi \frac{\partial v}{\partial t}\left(\tau_{n}\right)=\sum_{j=1}^{J} \frac{1}{\lambda_{j, n}^{N / 2}} \frac{\partial U_{\mathrm{L}}^{j}}{\partial t}\left(\frac{-t_{j, n}}{\lambda_{j, n}}, \frac{x-x_{j, n}}{\lambda_{j, n}}\right)+w_{1, n}(x),
\end{array}\right.
$$

where $U_{\mathrm{L}}^{j}$ is a solution of the linear wave equation 2.1 with initial conditions $\left(U_{0}^{j}, U_{1}^{j}\right)$. 
As $\psi(u-v)$ is supported in $\{|x| \leq 1-t\}$ when $t$ is close to 1 , Lemma2.5 implies

$$
\forall j \geq 1, \exists C_{j}, \forall n, \quad\left|\lambda_{j, n}\right|+\left|t_{j, n}\right|+\left|x_{j, n}\right| \leq C_{j}\left(1-\tau_{n}\right) .
$$

Let us first show:

Lemma 3.6. Reorder the decomposition (3.9) so that

$$
\left\|\nabla U_{0}^{1}\right\|_{L^{2}}^{2}+\left\|U_{1}^{1}\right\|_{L^{2}}^{2}=\sup _{j \geq 1}\left[\left\|\nabla U_{0}^{j}\right\|_{L^{2}}^{2}+\left\|U_{1}^{j}\right\|_{L^{2}}^{2}\right] .
$$

Then

$$
\left\|\nabla U_{0}^{1}\right\|_{L^{2}}^{2}+\left\|U_{1}^{1}\right\|_{L^{2}}^{2} \geq \frac{2}{N}\|\nabla W\|_{L^{2}}^{2}
$$

Lemma 3.6, together with the Pythagorean expansions 2.14 and 2.15, implies 3.5 immediately.

Remark 3.7. In space dimension $N=3$, we have the following immediate corollary of Lemma 3.6 , If

$$
\liminf _{t \rightarrow 1^{-}}\left[\|\nabla u(t)\|_{L^{2}}^{2}+\left\|\partial_{t} u(t)\right\|_{L^{2}}^{2}\right]<\frac{4}{3}\|\nabla W\|_{L^{2}}^{2}
$$

then there is only one singular point. See Remark 3.10 below for an improvement.

Proof of Lemma 3.6. Assume that

$$
\left\|\nabla U_{0}^{1}\right\|_{L^{2}}^{2}+\left\|U_{1}^{1}\right\|_{L^{2}}^{2}<\frac{2}{N}\|\nabla W\|_{L^{2}}^{2}
$$

and thus for all $j \geq 1$,

$$
\left\|\nabla U_{0}^{j}\right\|_{L^{2}}^{2}+\left\|U_{1}^{j}\right\|_{L^{2}}^{2}<\frac{2}{N}\|\nabla W\|_{L^{2}}^{2}
$$

Using that $2 E(f, g) \leq\|\nabla f\|_{L^{2}}^{2}+\|g\|_{L^{2}}^{2}$ and that $E(W, 0)=\frac{1}{N}\|\nabla W\|_{L^{2}}^{2}$, we find that there exists an $\varepsilon_{0}>0$ such that for all $j, n$,

$$
E\left(U_{\mathrm{L}}^{j}\left(-t_{j, n} / \lambda_{j, n}\right), \partial_{t} U_{\mathrm{L}}^{j}\left(-t_{j, n} / \lambda_{j, n}\right)\right) \leq E(W, 0)-\varepsilon_{0}, \quad\left\|\nabla U_{0}^{j}\right\|_{L^{2}}^{2} \leq\|\nabla W\|_{L^{2}}^{2}-\varepsilon_{0} .
$$

Then according to [KM08], for all $j, U^{j}$ is globally defined and scatters. By Proposition 2.8 the solution with initial condition $\left(\psi u\left(\tau_{n}\right), \psi \partial_{t} u\left(\tau_{n}\right)\right)$ is globally defined and scatters for large $n$. Using the finite speed of propagation, we get a contradiction with the fact that 0 is singular. Hence 3.12 follows.

It remains to show (3.4). We first recall the following scattering result (see [KM08, Corollary 7.4]) and [DKM]:

Proposition 3.8. Assume $N=3,4$. Let $u$ be a solution of 1.1 such that

$$
\limsup _{t \rightarrow T_{+}(u)}\left[\|\nabla u(t)\|_{L^{2}}^{2}+\left\|\partial_{t} u(t)\right\|_{L^{2}}^{2}\right]<\|\nabla W\|_{L^{2}}^{2} .
$$

Then $u$ is globally defined and scatters. 
The following proposition implies (3.4) by the Pythagorean expansions 2.14, (2.15):

Proposition 3.9. Assume $N=3$, 4. Let $\varepsilon_{0}>0$. There exists a sequence $\left\{\tilde{\tau}_{n}\right\} \in\left(t_{0}, 1\right)^{\mathbb{N}}$ that tends to 1 such that $\left(\psi a\left(\tilde{\tau}_{n}\right), \psi \partial_{t} a\left(\tilde{\tau}_{n}\right)\right)$ admits a profile decomposition $\left\{\widetilde{U}_{\mathrm{L}}^{j}\right\}_{j}$, $\left\{\tilde{\lambda}_{j, n}, \tilde{x}_{j, n}, \tilde{t}_{j, n}\right\}_{j, n}$ such that $\tilde{t}_{1, n}=0$ and

$$
\left\|\nabla \widetilde{U}_{0}^{1}\right\|_{L^{2}}^{2}+\left\|\widetilde{U}_{1}^{1}\right\|_{L^{2}}^{2} \geq\|\nabla W\|_{L^{2}}^{2}-\varepsilon_{0}
$$

Proof. We follow the proof of [KM08, Corollary 7.5]. In all the proof, we will always work up to extraction of a subsequence for sequences indexed by $n$. In particular, any real sequence indexed by $n$ will be assumed to have a limit in $\mathbb{R} \cup\{ \pm \infty\}$.

Select an increasing sequence $\left\{\tau_{n}\right\} \in\left(t_{0}, 1\right)^{\mathbb{N}}$ that tends to 1 . Let $\tilde{u}_{n}$ and $\tilde{v}_{n}$ be the solutions of (1.1) such that

$$
\left(\tilde{u}_{n}, \partial_{t} \tilde{u}_{n}\right)_{\mid t=\tau_{n}}=\left(\psi u\left(\tau_{n}\right), \psi \partial_{t} u\left(\tau_{n}\right)\right), \quad\left(\tilde{v}_{n}, \partial_{t} \tilde{v}_{n}\right)_{\left\lceil t=\tau_{n}\right.}=\left(\psi v\left(\tau_{n}\right), \psi \partial_{t} v\left(\tau_{n}\right)\right) .
$$

By finite speed of propagation and the fact that $x=0$ is a singular point for $u, T_{+}\left(\tilde{u}_{n}\right) \leq 1$. Furthermore, $\left(\psi v\left(\tau_{n}\right), \psi \partial_{t} v\left(\tau_{n}\right)\right)$ has a limit in $\dot{H}^{1} \times L^{2}$ as $n \rightarrow \infty$, which implies that there exists a small $t_{0}>0$ such that $\tilde{v}_{n}\left(\tau_{n}+t\right)$ is well-defined for large $n$ and $|t| \leq t_{0}$.

After extracting a subsequence, there exists a profile decomposition with profiles $\left\{U_{\mathrm{L}}^{j}\right\}$ and parameters $\left\{\lambda_{j, n}, x_{j, n}, t_{j, n}\right\}$ associated to the sequence $\left\{\left(\tilde{u}_{n}\left(\tau_{n}\right)-\tilde{v}_{n}\left(\tau_{n}\right)\right.\right.$, $\left.\left.\partial_{t} \tilde{u}_{n}\left(\tau_{n}\right)-\partial_{t} \tilde{v}_{n}\left(\tau_{n}\right)\right)\right\}_{n}$. The fact that $\tilde{\psi}(u-v)$ is supported in $\{|x| \leq 1-t\}$ and Lemma 2.5 imply

$$
\forall j \geq 1, \exists C_{j}, \forall n, \quad\left|\lambda_{j, n}\right|+\left|t_{j, n}\right|+\left|x_{j, n}\right| \leq C_{j}\left(1-\tau_{n}\right)
$$

Let us consider the associated nonlinear profiles $U^{j}$ (see Notation 2.6. Reordering the profiles, we get a $J_{0}$ such that

$$
\forall j \leq J_{0}, \quad\left\|U^{j}\right\|_{S\left(0, T_{+}\left(U^{j}\right)\right)}=\infty, \quad \forall j \geq J_{0}+1, \quad\left\|U^{j}\right\|_{S\left(0, T_{+}\left(U^{j}\right)\right)}<\infty .
$$

By the finite blow-up criterion, $T_{+}\left(U^{j}\right)=\infty$ if $j \geq J_{0}+1$. By Proposition 2.8 there is at least one solution $U^{j}$ that does not scatter forward in time (otherwise we would have $\left.T_{+}(u)>1\right)$, and thus $J_{0} \geq 1$.

For $1 \leq j \leq J_{0}, \lim _{n}-t_{j, n} / \lambda_{j, n}=\ell_{j} \in\{-\infty\} \cup \mathbb{R}$ (the case $\ell_{j}=\infty$ is excluded as the nonlinear profile does not scatter forward in time). If $\ell_{j}$ is finite, the corresponding profile is compact up to scaling and translation, and we may assume $t_{j, n}=0$. Thus

$$
\forall j \in\left\{1, \ldots, J_{0}\right\}, \quad t_{j, n}=0 \text { or } \lim _{n \rightarrow \infty} \frac{-t_{j, n}}{\lambda_{j, n}}=-\infty .
$$

By Proposition 3.8 , for all $j \in\left\{1, \ldots, J_{0}\right\}$, there exists a time $T_{j}$ such that

$$
T_{-}\left(U^{j}\right)<T_{j}<T_{+}\left(U^{j}\right) \quad \text { and } \quad\left\|\nabla u\left(T_{j}\right)\right\|_{L^{2}}^{2}+\left\|\partial_{t} u\left(T_{j}\right)\right\|_{L^{2}}^{2} \geq\|\nabla W\|_{L^{2}}^{2}-\varepsilon_{0},
$$

furthermore, using that $T_{+}\left(U^{j}\right)>0$ if $t_{j, n}=0$, we may choose $T_{j}$ such that

$$
\left(\forall n, t_{j, n}=0\right) \Rightarrow T_{j}>0 .
$$


Extracting subsequences and reordering the profiles, we may assume

$$
\forall n, \quad t_{1, n}+\lambda_{1, n} T_{1}=\min _{1 \leq j \leq J_{0}}\left(t_{j, n}+\lambda_{j, n} T_{j}\right) .
$$

Denote $\theta_{n}=t_{1, n}+\lambda_{1, n} T_{1}$. Note that $\theta_{n} \geq 0$ for large $n$ and, by 3.14,

$$
\lim _{n \rightarrow \infty} \theta_{n}=0 \text {. }
$$

For all $j$, we have, by definition of $\theta_{n},\left(\theta_{n}-t_{j, n}\right) / \lambda_{j, n} \leq T_{j}<T_{+}\left(U^{j}\right)$. According to Remark 2.9 we can use Proposition 2.8 which shows that $\tau_{n}+\theta_{n}<T_{+}\left(\tilde{u}_{n}\right) \leq 1$, that $\left\{\left\|\tilde{u}_{n}\right\|_{S\left(\tau_{n}, \tau_{n}+\theta_{n}\right)}\right\}_{n}$ is bounded and

$$
\begin{aligned}
\tilde{u}_{n}\left(\tau_{n}+t\right)= & \tilde{v}_{n}\left(\tau_{n}+t\right)+\sum_{j=1}^{J} \frac{1}{\lambda_{j, n}^{(N-2) / 2}} U^{j}\left(\frac{t-t_{j, n}}{\lambda_{j, n}}, \frac{x-x_{j, n}}{\lambda_{j, n}}\right) \\
& +w_{n}^{J}(t, x)+r_{n}^{J}(t, x), \quad t \in\left(0, \theta_{n}\right),
\end{aligned}
$$

where $r_{n}^{J}$ satisfies 2.23). If $j \geq 1$, there exists (extracting a subsequence if necessary) a linear wave $\tilde{U}_{\mathrm{L}}^{j}$ such that

$$
\begin{aligned}
\lim _{n \rightarrow \infty}\left[\| U^{j}\left(\frac{\theta_{n}-t_{j, n}}{\lambda_{j, n}}\right)-\right. & \widetilde{U}_{\mathrm{L}}^{j}\left(\frac{\theta_{n}-t_{j, n}}{\lambda_{j, n}}\right) \|_{\dot{H}^{1}} \\
& \left.+\left\|\partial_{t} U^{j}\left(\frac{\theta_{n}-t_{j, n}}{\lambda_{j, n}}\right)-\partial_{t} \widetilde{U}_{\mathrm{L}}^{j}\left(\frac{\theta_{n}-t_{j, n}}{\lambda_{j, n}}\right)\right\|_{L^{2}}\right]=0 .
\end{aligned}
$$

Indeed, if $\left\{\left(\theta_{n}-t_{j, n}\right) / \lambda_{j, n}\right\}_{n}$ converges this is obvious, and if it goes to $-\infty$, then also $\left\{\left(\theta_{n}-t_{j, n}\right) / \lambda_{j, n}\right\}_{n}$ goes to $-\infty$, and we can take $\tilde{U}_{\mathrm{L}}^{j}=U_{\mathrm{L}}^{j}$. Writing $\tilde{\tau}_{n}=\tau_{n}+\theta_{n}$ and $\tilde{t}_{j, n}=t_{j, n}-\theta_{n}$ we get by 3.19,

$$
\left\{\begin{array}{l}
\left(\tilde{u}_{n}-\tilde{v}_{n}\right)\left(\tilde{\tau}_{n}\right)=\sum_{j=1}^{J} \frac{1}{\lambda_{j, n}^{N / 2-1}} \tilde{U}_{\mathrm{L}}^{j}\left(\frac{-\tilde{t}_{j, n}}{\lambda_{j, n}}, \frac{x-x_{j, n}}{\lambda_{j, n}}\right)+w_{n}^{J}\left(\theta_{n}\right)+r_{n}^{J}\left(\theta_{n}\right)+o_{n}(1), \\
\partial_{t}\left(\tilde{u}_{n}-\tilde{v}_{n}\right)\left(\tilde{\tau}_{n}\right)=\sum_{j=1}^{J} \frac{1}{\lambda_{j, n}^{N / 2}} \partial_{t} \tilde{U}_{\mathrm{L}}^{j}\left(\frac{-\tilde{t}_{j, n}}{\lambda_{j, n}}, \frac{x-x_{j, n}}{\lambda_{j, n}}\right)+\partial_{t} w_{n}^{J}\left(\theta_{n}\right)+\partial_{t} r_{n}^{J}\left(\theta_{n}\right)+o_{n}(1),
\end{array}\right.
$$

This is a profile decomposition for the sequence $\left\{\left(\tilde{u}_{n}\left(\tilde{\tau}_{n}\right)-\tilde{v}_{n}\left(\tilde{\tau}_{n}\right), \partial_{t} \tilde{u}_{n}\left(\tilde{\tau}_{n}\right)-\partial_{t} \tilde{v}_{n}\left(\tilde{\tau}_{n}\right)\right)\right\}$, with profiles $\left\{\tilde{U}_{\mathrm{L}}^{j}\right\}$ and parameters $\left\{\lambda_{j, n}, x_{j, n}, \tilde{t}_{j, n}\right\}$. Note that the orthogonality of the parameters follows directly from the equality $\tilde{t}_{j, n}-\tilde{t}_{k, n}=t_{j, n}-t_{k, n}$.

Next notice that by finite speed of propagation and the definitions of $\tilde{u}_{n}$ and $\tilde{v}_{n}$, there exists an $r_{0}>0$ such that if $n$ is large and $|x|<r_{0}$ then $\tilde{u}_{n}\left(\tilde{\tau}_{n}\right)=u\left(\tilde{\tau}_{n}\right), \partial_{t} \tilde{u}_{n}\left(\tilde{\tau}_{n}\right)=$ $\partial_{t} u\left(\tilde{\tau}_{n}\right), \tilde{v}_{n}\left(\tilde{\tau}_{n}\right)=v\left(\tilde{\tau}_{n}\right)$ and $\partial_{t} \tilde{v}_{n}\left(\tilde{\tau}_{n}\right)=\partial_{t} v\left(\tilde{\tau}_{n}\right)$. Using that $u\left(\tilde{\tau}_{n}\right)-v\left(\tilde{\tau}_{n}\right)$ and $\partial_{t} u\left(\tilde{\tau}_{n}\right)-$ $\partial_{t} v\left(\tilde{\tau}_{n}\right)$ are supported in the set $\left\{|x| \leq 1-\tilde{\tau}_{n}\right\}$, one can replace, in the decomposition (3.20), $\tilde{u}_{n}$ and $\tilde{v}_{n}$ by $\psi u$ and $\psi v$.

Finally, $\left(\theta_{n}-t_{1, n}\right) / \lambda_{1, n}=T_{1}$. Thus the first profile $\widetilde{U}^{1}$ in the decomposition 3.20 is compact up to modulation, and we may assume $\tilde{t}_{1, n}=0$ as announced. The inequality (3.13) follows from the choice of $T_{1}$. 
Remark 3.10. In space dimension $N=3$, 4, we can improve Remark 3.7 as follows. If for some $t_{0} \in(0,1)$,

$$
\sup _{t \in\left(t_{0}, 1\right)}\left[\|\nabla u(t)\|_{L^{2}}^{2}+\left\|\partial_{t} u(t)\right\|_{L^{2}}^{2}\right]<\left(1+\frac{2}{N}\right)\|\nabla W\|_{L^{2}}^{2},
$$

then there is only one singular point. This is a direct consequence of 3.4 and 3.5 .

\section{Finite speed of propagation and exclusion of small exterior profiles}

In the next two sections, we assume that $N=3$ and that $u$ is spherically symmetric, blows up at time $T=1$ and satisfies

$$
\sup _{\tau_{0} \leq t<1} \sqrt{\|\nabla u(t)\|_{L^{2}}^{2}+\left\|\partial_{t} u(t)\right\|_{L^{2}}^{2}} \leq C_{0}
$$

In these two sections we will not make any further assumption on $C_{0}>0$. By spherical symmetry, 0 is the only singular point. We denote by

$$
a(t, x)=u(t, x)-v(t, x)
$$

the singular part of $u$ at the blow-up time $t=1$ (see Definition 3.3.

The main result of this section (Proposition 4.4, shown in $\$ 4.2$, is that the norm of the most exterior profile of any profile decomposition of a sequence $\left\{\left(a\left(t_{n}\right), \partial_{t} a\left(t_{n}\right)\right)\right\}$ is bounded from below by a universal constant independent of the solution.

\subsection{Linear behavior}

We start by two preliminary results on the linear problem, valid in odd dimension only, that will be needed later. The first one follows from the Huyghens principle:

Lemma 4.1. Assume that $N$ is odd. Let $v$ be a solution of the linear wave equation (2.1) with initial conditions $\left(v_{0}, v_{1}\right)$, let $\left\{\lambda_{n}\right\}_{n},\left\{t_{n}\right\}_{n}$ be two real sequences with $\lambda_{n}$ positive, set

$$
v_{n}(t, x)=\frac{1}{\lambda_{n}^{N / 2-1}} v\left(\frac{t}{\lambda_{n}}, \frac{x}{\lambda_{n}}\right)
$$

and assume $\lim _{n \rightarrow \infty} t_{n} / \lambda_{n}=\ell \in[-\infty, \infty]$. Then, if $\ell= \pm \infty$,

$$
\lim _{R \rightarrow \infty} \limsup _{n \rightarrow \infty} \int_{|| x|-| t_{n}|| \geq R \lambda_{n}}\left[\left|\nabla v_{n}\left(t_{n}\right)\right|^{2}+\frac{1}{|x|^{2}}\left|v_{n}\left(t_{n}\right)\right|^{2}+\left(\partial_{t} v_{n}\left(t_{n}\right)\right)^{2}\right] d x=0,
$$

and if $\ell \in \mathbb{R}$,

$$
\lim _{R \rightarrow \infty} \limsup _{n \rightarrow \infty} \int_{\substack{\left\{|x| \geq R \lambda_{n}\right\} \\ \cup\left\{|x| \leq \lambda_{n} / R\right\}}}\left[\left|\nabla v_{n}\left(t_{n}\right)\right|^{2}+\frac{1}{|x|^{2}}\left|v_{n}\left(t_{n}\right)\right|^{2}+\left(\partial_{t} v_{n}\left(t_{n}\right)\right)^{2}\right] d x=0 .
$$


Proof. This is a classical property. In the case $\ell \in \mathbb{R}$, just notice that

$$
\begin{aligned}
& v_{n}\left(t_{n}, x\right)=\frac{1}{\lambda_{n}^{N / 2-1}} v\left(\ell, \frac{x}{\lambda_{n}}\right)+o_{n}(1) \text { in } \dot{H}^{1}, \\
& \partial_{t} v_{n}\left(t_{n}, x\right)=\frac{1}{\lambda_{n}^{N / 2}} \partial_{t} v\left(\ell, \frac{x}{\lambda_{n}}\right)+o_{n}(1) \quad \text { in } L^{2} \text {, }
\end{aligned}
$$

which implies the announced estimate (in this case we do not need any assumption on the parity of $N$ ).

Let us treat the case $\ell= \pm \infty$. Let $\varepsilon>0$, and let $\chi \in C_{0}^{\infty}\left(\mathbb{R}^{N}\right)$ be such that $\chi(x)=1$ for $|x| \leq 1 / 2$ and $\chi(x)=0$ for $|x| \geq 1$. Then

$$
\lim _{R \rightarrow \infty}\left[\left\|\nabla\left(v_{0}^{R}-v_{0}\right)\right\|_{L^{2}}+\left\|v_{1}^{R}-v_{1}\right\|_{L^{2}}\right]=0
$$

where

$$
v_{0}^{R}(x)=\chi(x / R) v_{0}(x), \quad v_{1}^{R}(x)=\chi(x / R) v_{1}(x) .
$$

Choose $R_{\varepsilon}$ such that for $R \geq R_{\varepsilon}, \sqrt{\left\|\nabla\left(v_{0}^{R}-v_{0}\right)\right\|_{L^{2}}^{2}+\left\|v_{1}^{R}-v_{1}\right\|_{L^{2}}^{2}} \leq \varepsilon$. Let $R \geq R_{\varepsilon}$ and denote by $v_{n}^{R}$ the solution with initial condition $v_{0, n}^{R}=\lambda_{n}^{1-N / 2} v_{0}^{R}\left(x / \lambda_{n}\right), v_{1, n}^{R}=$ $\lambda_{n}^{-N / 2} v_{1}^{R}\left(x / \lambda_{n}\right)$. By conservation of energy and the scaling of the equation,

$$
\forall n, \quad \sqrt{\left\|\nabla v_{n}^{R}\left(t_{n}\right)-\nabla v_{n}\left(t_{n}\right)\right\|_{L^{2}}^{2}+\left\|\partial_{t} v_{n}^{R}\left(t_{n}\right)-\partial_{t} v_{n}\left(t_{n}\right)\right\|_{L^{2}}^{2}} \leq \varepsilon .
$$

By the strong Huyghens principle, $\left(v_{n}^{R}\left(t_{n}\right), \partial_{t} v_{n}^{R}\left(t_{n}\right)\right)$ is supported in the ring $\left\{\left|t_{n}\right|-\right.$ $\left.R \lambda_{n} \leq|x| \leq\left|t_{n}\right|+R \lambda_{n}\right\}$. Hence for large $n$ (using Hardy's inequality),

$$
\begin{aligned}
& \left(\int_{|| t_{n}|-| x|| \geq R \lambda_{n}}\left[\left|\nabla v_{n}\left(t_{n}\right)\right|^{2}+\frac{1}{|x|^{2}}\left|v_{n}\left(t_{n}\right)\right|^{2}+\left(\partial_{t} v_{n}\left(t_{n}\right)\right)^{2}\right] d x\right)^{1 / 2} \\
& \quad \leq\left(\int_{|| t_{n}|-| x|| \geq R \lambda_{n}}\left[\left|\nabla v_{n}^{R}\left(t_{n}\right)\right|^{2}+\frac{1}{|x|^{2}}\left|v_{n}^{R}\left(t_{n}\right)\right|^{2}+\left(\partial_{t} v_{n}^{R}\left(t_{n}\right)\right)^{2}\right] d x\right)^{1 / 2}+C \varepsilon=C \varepsilon,
\end{aligned}
$$

which concludes the proof of the lemma.

We next give, in Lemma 4.2, a property of the energy of radial solutions to the linear equation in space dimension $N=3$. In Corollary 4.3 we deduce a similar property for solutions of the nonlinear equation which are sums of small profiles.

Lemma 4.2. Assume that $N=3$. Let $v$ be a radial solution of 2.1 , $t_{0} \in \mathbb{R}, 0<r_{0}<r_{1}$. Then the following property holds for all $t \geq t_{0}$ or for all $t \leq t_{0}$ :

$$
\begin{aligned}
\int_{r_{0}+\left|t-t_{0}\right|<r<r_{1}+\left|t-t_{0}\right|}\left[\left(\partial_{r}(r v(t, x))\right)^{2}+r^{2}\left(\partial_{t} v(t, x)\right)^{2}\right] d r \\
\quad \geq \frac{1}{2} \int_{r_{0}<r<r_{1}}\left[\left(\partial_{r}\left(r v\left(t_{0}, x\right)\right)\right)^{2}+r^{2}\left(\partial_{t} v\left(t_{0}, x\right)\right)^{2}\right] d r .
\end{aligned}
$$


Proof. We can assume that $t_{0}=0$. Let $f=r v, f_{0}=f_{\mid t=0}, f_{1}=\partial_{t} f_{\mid t=0}$. Then

$$
\partial_{t}^{2} f=\partial_{r}^{2} f, \quad t \in \mathbb{R}, r>0 .
$$

Furthermore, as $v(t)$ is in $\dot{H}^{1}$ for all $t$, by Hardy's inequality in dimension 3 ,

$$
\int \frac{1}{r^{2}}(f(t, r))^{2} d r+\int\left(\partial_{r} f(t, r)\right)^{2} d r<\infty .
$$

By Sobolev embeddings in dimension 1 , for all $t, f(t, \cdot)$ is continuous and satisfies the condition $f(t, 0)=0$. By explicit computation we get

$$
f(t, r)=F(t+r)-F(t-r), \quad t \in \mathbb{R}, r>0,
$$

where $F$ is defined by

$$
F(s)= \begin{cases}\frac{1}{2} f_{0}(s)+\frac{1}{2} \int_{0}^{s} f_{1}(\sigma) d \sigma, & s>0, \\ -\frac{1}{2} f_{0}(-s)+\frac{1}{2} \int_{0}^{-s} f_{1}(\sigma) d \sigma, & s<0 .\end{cases}
$$

Thus, if $t \in \mathbb{R}$,

$$
\int_{r_{0}+|t|}^{r_{1}+|t|}\left[\left(\partial_{t} f(t, r)\right)^{2}+\left(\partial_{r} f(t, r)\right)^{2}\right] d r=2 \int_{r_{0}+|t|}^{r_{1}+|t|}\left[\left(F^{\prime}(t+r)\right)^{2}+\left(F^{\prime}(t-r)\right)^{2}\right] d r .
$$

Consequently, if $t>0$,

$$
\int_{r_{0}+|t|}^{r_{1}+|t|}\left[\left(\partial_{t} f\right)^{2}+\left(\partial_{r} f\right)^{2}\right] d r \geq 2 \int_{r_{0}}^{r_{1}}\left(F^{\prime}(-r)\right)^{2} d r
$$

and if $t<0$,

$$
\int_{r_{0}+|t|}^{r_{1}+|t|}\left[\left(\partial_{t} f\right)^{2}+\left(\partial_{r} f\right)^{2}\right] d r \geq 2 \int_{r_{0}}^{r_{1}}\left(F^{\prime}(r)\right)^{2} d r .
$$

By (4.4) at $t=0$ we see that

$$
\int_{r_{0}+|t|}^{r_{1}+|t|}\left[\left(\partial_{t} f(t, r)\right)^{2}+\left(\partial_{r} f(t, r)\right)^{2}\right] d r \geq \frac{1}{2} \int_{r_{0}}^{r_{1}}\left[\left(f_{1}(r)\right)^{2}+\left(\partial_{r} f_{0}(r)\right)^{2}\right] d r
$$

for all $t>0$ or for all $t<0$, hence (4.2) follows.

Corollary 4.3. Assume that $N=3$. Let $C_{0}>0$. Then there exists a constant $\delta_{1}=$ $\delta_{1}\left(C_{0}\right)>0$ with the following property. Let $J>0$, and let $\left\{\lambda_{1, n}\right\}_{n}, \ldots,\left\{\lambda_{J, n}\right\}_{n}$ be sequences of positive numbers such that

$$
\lambda_{1, n} \ll \cdots \ll \lambda_{J, n} \quad \text { as } n \rightarrow \infty .
$$


Pick $J$ radial solutions $U^{1}, \ldots, U^{J}$ of 1.1 with initial conditions $\left(U_{0}^{j}, U_{1}^{j}\right), j=$ $1, \ldots, J$, such that

$$
\forall j \in\{1, \ldots, J\}, \quad \sqrt{\left\|\nabla U_{0}^{j}\right\|_{L^{2}}^{2}+\left\|U_{1}^{j}\right\|_{L^{2}}^{2}}=\eta_{j} \leq \delta_{1} .
$$

Select a sequence $\left\{w_{n}\right\}$ of solutions of the linear wave equation 2.1 such that

$\forall j \in\{1, \ldots, J\}, \quad\left(\lambda_{j}^{N / 2-1} w_{0, n}\left(\lambda_{j} x\right), \lambda_{j}^{N / 2} w_{1, n}\left(\lambda_{j} x\right)\right) \underset{n \rightarrow \infty}{\longrightarrow} 0$ weakly in $\dot{H}^{1} \times L^{2}$,

Let $\eta=\sqrt{\sum_{j=1}^{J} \eta_{j}^{2}}$ and assume that $\eta \leq C_{0}$. Let

$$
U_{n}(t, x)=\sum_{j=1}^{J} \frac{1}{\lambda_{j, n}^{N / 2-1}} U^{j}\left(\frac{t}{\lambda_{j, n}}, \frac{x}{\lambda_{j, n}}\right)+w_{n}(t, x) .
$$

Then there exists $r_{1}>0$ such that for large $n$, the inequality

$$
\sqrt{\int_{r_{1} \lambda_{1, n}+|t|<|x|}\left[\left|\nabla U_{n}(t, x)\right|^{2}+\left(\partial_{t} U_{n}(t, x)\right)^{2}\right] d x} \geq \frac{\eta}{4}
$$

holds for all $t>0$ or for all $t<0$.

Proof. Denote

$$
U_{0, n}(x)=U_{n}(0, x), \quad U_{1, n}(x)=\partial_{t} U_{n}(0, x) .
$$

Let $U_{\mathrm{L}}^{j}$ be the solution of 2.1 with initial conditions $\left(U_{0}^{j}, U_{1}^{j}\right), j=1, \ldots, J$, and

$$
U_{\mathrm{L}, n}(t, x)=\sum_{j=1}^{J} \frac{1}{\lambda_{j, n}^{N / 2-1}} U_{\mathrm{L}}^{j}\left(\frac{t}{\lambda_{j, n}}, \frac{x}{\lambda_{j, n}}\right)+w_{n}(t, x) .
$$

Step 1. We first show that if $\delta_{1}=\delta_{1}\left(C_{0}\right)$ is chosen small enough, then

$$
\sup _{t \in \mathbb{R}} \sqrt{\left\|U_{n}(t)-U_{\mathrm{L}, n}(t)\right\|_{\dot{H}^{1}}^{2}+\left\|\partial_{t} U_{n}(t)-\partial_{t} U_{\mathrm{L}, n}(t)\right\|_{L^{2}}^{2}} \leq \frac{\eta}{4} .
$$

Indeed by (2.8), if $\delta_{1}^{3} \leq 1 /\left(C C_{0}\right)$ for some large constant $C$, then $\sqrt{\left\|\nabla U_{0}^{j}\right\|_{L^{2}}^{2}+\left\|U_{1}^{j}\right\|_{L^{2}}^{2}}$ $=\eta_{j} \leq \delta_{1}$ implies

$$
\sup _{t \in \mathbb{R}} \sqrt{\left\|U^{j}(t)-U_{\mathrm{L}}^{j}(t)\right\|_{\dot{H}^{1}}^{2}+\left\|\partial_{t} U^{j}(t)-\partial_{t} U_{\mathrm{L}}^{j}(t)\right\|_{L^{2}}^{2}} \leq C \eta_{j}^{5} \leq \frac{\eta_{j}^{2}}{4 C_{0}} .
$$

By the triangle inequality and the fact that $\eta \leq C_{0}$,

$$
\sup _{t \in \mathbb{R}} \sqrt{\left\|U_{n}(t)-U_{\mathrm{L}, n}(t)\right\|_{\dot{H}^{1}}^{2}+\left\|\partial_{t} U_{n}(t)-\partial_{t} U_{\mathrm{L}, n}(t)\right\|_{L^{2}}^{2}} \leq \frac{\eta^{2}}{4 C_{0}} \leq \frac{\eta}{4} .
$$

Hence 4.6 follows. 
Step 2. We next show that there exists $r_{1}>0$ such that

$$
\liminf _{n \rightarrow \infty} \int_{r_{1} \lambda_{1, n}}^{\infty}\left[\left(\partial_{r}\left(r U_{0, n}(r)\right)\right)^{2}+\left(r U_{1, n}(r)\right)^{2}\right] d r \geq \frac{\eta^{2}}{2} .
$$

Indeed, if $f \in \dot{H}^{1}$ is a radial function and $0<R_{0}<R_{1}$,

$$
\begin{aligned}
\int_{R_{0}}^{R_{1}}\left(\partial_{r}(r f(r))\right)^{2} d r & =\int_{R_{0}}^{R_{1}}\left[f^{2}+r^{2}\left(\partial_{r} f\right)^{2}+2 r f \partial_{r} f\right] d r \\
& =\int_{R_{0}}^{R_{1}}\left[f^{2}+r^{2}\left(\partial_{r} f\right)^{2}+r \partial_{r}\left(f^{2}\right)\right] d r \\
& =\int_{R_{0}}^{R_{1}} r^{2}\left(\partial_{r} f\right)^{2} d r+R_{1} f^{2}\left(R_{1}\right)-R_{0} f^{2}\left(R_{0}\right) .
\end{aligned}
$$

By Hardy's inequality, $\int f(t, r)^{2} d r<\infty$, which implies that there exist sequences $R_{n} \rightarrow \infty$ and $\tilde{R}_{n} \rightarrow 0$ such that $R_{n} f\left(R_{n}\right)^{2} \rightarrow 0$ and $\tilde{R}_{n} f\left(\tilde{R}_{n}\right)^{2} \rightarrow 0$. Letting $R_{1}=R_{n}$ and $n \rightarrow \infty$, we get

$$
\int_{R_{0}}^{\infty}\left(\partial_{r}(r f(r))\right)^{2} d r=\int_{|x| \geq R_{0}}|\nabla f|^{2} d x-R_{0} f\left(R_{0}\right)^{2} \leq \int_{|x| \geq R_{0}}|\nabla f|^{2} d x .
$$

Letting $R_{0}=\tilde{R}_{n}$ and $n \rightarrow \infty$ we get

$$
\int_{0}^{\infty}\left(\partial_{r}(r f(r))\right)^{2} d r=\int_{\mathbb{R}^{3}}|\nabla f|^{2} d x .
$$

By 4.9), there exists $r_{1}>0$ such that

$$
\int_{r_{1}}^{\infty}\left[\left(\partial_{r}\left(r U_{0}^{1}(r)\right)\right)^{2}+\left(r U_{1}^{1}(r)\right)^{2}\right] d r \geq \frac{\eta_{1}^{2}}{2} .
$$

Let $g^{j}=\partial_{r}\left(r U_{0}^{j}(r)\right) \in L^{2}(d r)$. Then

$$
\begin{aligned}
A_{n}^{j, k} & :=\left|\int_{r_{1} \lambda_{1, n}}^{\infty} \partial_{r}\left(\frac{r}{\lambda_{j, n}^{1 / 2}} U_{0}^{j}\left(\frac{r}{\lambda_{j, n}}\right)\right) \partial_{r}\left(\frac{r}{\lambda_{k, n}^{1 / 2}} U_{0}^{k}\left(\frac{r}{\lambda_{k, n}}\right)\right) d r\right| \\
& =\left|\int_{r_{1} \lambda_{1, n}}^{\infty} \frac{1}{\lambda_{j, n}^{1 / 2}} g_{j}\left(\frac{r}{\lambda_{j, n}}\right) \frac{1}{\lambda_{k, n}^{1 / 2}} g_{k}\left(\frac{r}{\lambda_{k, n}}\right) d r\right| \\
& =\left|\int_{r_{1} \lambda_{1, n} / \lambda_{j, n}}^{\infty} g_{j}(\rho) \frac{\lambda_{j, n}^{1 / 2}}{\lambda_{k, n}^{1 / 2}} g_{k}\left(\frac{\lambda_{j, n}}{\lambda_{k, n}} \rho\right) d \rho\right| .
\end{aligned}
$$

Letting $j=k$ in 4.11) we deduce that if $j>1$, then $A_{n}^{j, j} \rightarrow \int_{0}^{\infty} g_{j}(\rho)^{2} d \rho$ as $n \rightarrow \infty$. Furthermore if $1 \leq j<k$, using that $\lambda_{k, n} / \lambda_{j, n} \rightarrow \infty$, we find that for all $\varepsilon>0$,

$$
\left|A_{n}^{j, k}\right| \leq C_{j} \sqrt{\int_{0}^{R_{\varepsilon}} \frac{\lambda_{j, n}}{\lambda_{k, n}}\left(g_{k}\left(\frac{\lambda_{j, n}}{\lambda_{k, n}} \rho\right)\right)^{2} d \rho}+C_{k} \sqrt{\int_{R_{\varepsilon}}^{\infty} g_{j}(\rho)^{2} d \rho} \leq o_{n}(1)+\varepsilon .
$$


and hence $A_{n}^{j, k} \rightarrow 0$ as $n \rightarrow \infty$. Similarly, noting that $h_{n}=\partial_{r}\left(r w_{0, n}\right)=w_{0, n}+r \partial_{r} w_{0, n}$ is such that $\lambda_{j, n}^{1 / 2} h_{n}\left(\lambda_{j, n} \cdot\right)$ converges weakly to 0 in $L^{2}(d r)$ we get

$$
\begin{aligned}
\int_{r_{1} \lambda_{1, n}}^{\infty} \partial_{r}\left(\frac{r}{\lambda_{j, n}^{1 / 2}} U_{0}^{j}\left(\frac{r}{\lambda_{j, n}}\right)\right) \partial_{r}\left(r w_{0, n}\right) d r=\int_{r_{1} \lambda_{1, n} / \lambda_{j, n}}^{\infty} g_{j}(\rho) \lambda_{j, n}^{1 / 2} h_{n}\left(\lambda_{j, n} \rho\right) d \rho \\
=\left\{\begin{array}{l}
\int_{r_{1}}^{\infty} g_{1}(\rho) \lambda_{1, n}^{1 / 2} h_{n}\left(\lambda_{1, n} \rho\right) d \rho+o_{n}(1) \quad \text { if } j=1, \\
\int_{0}^{\infty} g_{j}(\rho) \lambda_{j, n}^{1 / 2} h_{n}\left(\lambda_{j, n} \rho\right) d \rho+o_{n}(1) \quad \text { if } j>1,
\end{array}\right.
\end{aligned}
$$

which tends to 0 as $n \rightarrow \infty$. Using similar estimates on $U_{1, n}$ and $w_{1, n}$ and combining with (4.10) we get 4.7).

Step 3: End of the proof. In view of Step 2 and Lemma 4.2 if $n$ is large, then the following holds for all $t>0$ or for all $t<0$ :

$$
\int_{r_{1} \lambda_{1, n}+|t|}^{\infty}\left[\left(\partial_{r}\left(r U_{n, \mathrm{~L}}\right)\right)^{2}+\left(\partial_{t}\left(r U_{n, \mathrm{~L}}\right)\right)^{2}\right] d r \geq \frac{\eta^{2}}{4} .
$$

By [4.8, for all $t>0$ or for all $t<0$,

$$
\int_{|x| \geq r_{1} \lambda_{1, n}+|t|}\left[\left|\nabla U_{n, \mathrm{~L}}\right|^{2}+\left|\partial_{t} U_{n, \mathrm{~L}}\right|^{2}\right] d x \geq \frac{\eta^{2}}{4} .
$$

By Step 1 and the triangle inequality,

$$
\sqrt{\int_{|x| \geq r_{1} \lambda_{1, n}+|t|}\left[\left|\nabla U_{n}\right|^{2}+\left|\partial_{t} U_{n}\right|^{2}\right] d x} \geq \frac{\eta}{2}-\frac{\eta}{4}=\frac{\eta}{4},
$$

which concludes the proof.

\subsection{No small exterior profile}

Before stating the main result of this section, we introduce some notation. Throughout the following, we assume $N=3$. Let $\tau_{n} \rightarrow 1^{-}$and consider a profile decomposition of $\left\{\left(a\left(\tau_{n}\right), \partial_{t} a\left(\tau_{n}\right)\right)\right\}$ with profiles $\left\{U_{\mathrm{L}}^{j}\right\}$ and parameters $\left\{\lambda_{j, n}, t_{j, n}\right\}$. We will consider as usual the nonlinear profiles $\left\{U^{j}\right\}$ associated to $\left\{U_{\mathrm{L}}^{j}\right\},-t_{j, n} / \lambda_{j, n}$, and will write, for the sake of simplicity,

$$
U_{\mathrm{L}, n}^{j}(t, x)=\frac{1}{\lambda_{j, n}^{1 / 2}} U_{\mathrm{L}}^{j}\left(\frac{t-t_{j, n}}{\lambda_{j, n}}, \frac{x}{\lambda_{j, n}}\right), \quad U_{n}^{j}(t, x)=\frac{1}{\lambda_{j, n}^{3 / 2}} U^{j}\left(\frac{t-t_{j, n}}{\lambda_{j, n}}, \frac{x}{\lambda_{j, n}}\right) .
$$

The second expression is defined as long as $\left(t-t_{j, n}\right) / \lambda_{j, n}$ is in $\left(T_{-}\left(U^{j}\right), T_{+}\left(U^{j}\right)\right)$. We will also write

$$
U_{0, n}^{j}=U_{\mathrm{L}, n}^{j}(0, x), \quad U_{1, n}^{j}=\left(\partial_{t} U_{\mathrm{L}, n}^{j}\right)(0, x) .
$$


Let $j \in \mathbb{N}^{*}$. Extracting subsequences and time-translating the profiles if necessary we can assume that

$$
\forall n, t_{j, n}=0 \quad \text { or } \quad \lim _{n \rightarrow \infty} \frac{t_{j, n}}{\lambda_{j, n}} \in\{-\infty, \infty\} .
$$

We define

$$
\rho_{j, n}= \begin{cases}\left|t_{j, n}\right| & \text { if }\left|t_{j, n}\right| / \lambda_{j, n} \rightarrow \infty \\ \lambda_{j, n} & \text { if } t_{j, n}=0\end{cases}
$$

According to Lemma 4.1 the sequence $\left\{\left(U_{0, n}^{j}, U_{1, n}^{j}\right)\right\}_{n}$ is localized, for large $n$, around $|x| \approx \rho_{j, n}$.

Reordering the profiles and extracting subsequences, we can find a $J_{0} \in \mathbb{N}$ such that (here $\delta_{1}\left(C_{0}\right)$ is given by Corollary 4.3, and $C_{0}$ is the constant in assumption (4.1))

$$
\begin{aligned}
j>J_{0} \Leftrightarrow & \left(E\left(U_{0}^{j}, U_{1}^{j}\right) \leq\left(\delta_{1}\left(C_{0}\right)\right)^{2} / N, \text { and }\left\|\nabla U_{0}^{j}\right\|_{L^{2}}<\|\nabla W\|_{L^{2}}\right) \\
& \text { or }\left(\lim _{n \rightarrow \infty}-t_{j, n} / \lambda_{j, n} \in\{ \pm \infty\} \text { and } E\left(U_{0}^{j}, U_{1}^{j}\right)<E(W, 0)\right), \\
\rho_{J_{0}, n} \lesssim & \rho_{J_{0}-1, n} \lesssim \cdots \lesssim \rho_{1, n} .
\end{aligned}
$$

In particular if $j>J_{0}$ and $t_{j, n}=0$ for all $n$, by Claim 2.3 we have $\left\|\nabla U_{0}^{j}\right\|_{L^{2}}^{2}+\left\|U_{1}^{j}\right\|_{L^{2}}^{2} \leq$ $\left(\delta_{1}\left(C_{0}\right)\right)^{2}$.

In this section we show:

Proposition 4.4. Under the above assumptions,

$$
\lim _{R \rightarrow \infty} \limsup _{n \rightarrow \infty} \int_{|x| \geq R \rho_{1, n}}\left[\left|\nabla a\left(\tau_{n}\right)\right|^{2}+\left(\partial_{t} a\left(\tau_{n}\right)\right)^{2}\right] d x=0 .
$$

Proof. If (4.15) does not hold, there exists $\varepsilon_{0}>0$ and a sequence $\{\bar{\rho}\}_{n}$ such that

$$
\int_{|x| \geq \bar{\rho}_{n}}\left[\left|\nabla a\left(\tau_{n}\right)\right|^{2}+\left(\partial_{t} a\left(\tau_{n}\right)\right)^{2}\right] d x \geq \varepsilon_{0}, \quad \lim _{n \rightarrow \infty} \frac{\bar{\rho}_{n}}{\rho_{1, n}}=\infty .
$$

Since supp $a\left(\tau_{n}\right) \subset\left\{|x| \leq 1-\tau_{n}\right\}$, we have $\bar{\rho}_{n} \leq 1-\tau_{n}$. Moreover, by Claim B.1. we get, extracting subsequences in $n$, a sequence $\left\{\tilde{\rho}_{n}\right\}_{n}$ such that

$$
\rho_{1, n} \ll \tilde{\rho}_{n} \ll \bar{\rho}_{n}
$$

and

$$
\forall j, \quad \tilde{\rho}_{n} \ll \rho_{j, n} \text { or } \rho_{j, n} \ll \tilde{\rho}_{n} .
$$

Let $\chi \in C^{\infty}\left(\mathbb{R}^{N}\right)$ be such that $\chi(x)=1$ if $|x| \geq 2$ and $\chi(x)=0$ if $|x| \leq 1$. Then

$$
\begin{gathered}
\chi\left(x / \tilde{\rho}_{n}\right) u\left(\tau_{n}, x\right)=\chi\left(x / \tilde{\rho}_{n}\right) v\left(\tau_{n}, x\right)+\sum_{j=1}^{J} \chi\left(x / \tilde{\rho}_{n}\right) U_{0, n}^{j}+\chi\left(x / \tilde{\rho}_{n}\right) w_{0, n}^{J}, \\
\chi\left(x / \tilde{\rho}_{n}\right) \partial_{t} u\left(\tau_{n}, x\right)=\chi\left(x / \tilde{\rho}_{n}\right) \partial_{t} v\left(\tau_{n}, x\right)+\sum_{j=1}^{J} \chi\left(x / \tilde{\rho}_{n}\right) U_{1, n}^{j}+\chi\left(x / \tilde{\rho}_{n}\right) w_{1, n}^{J} .
\end{gathered}
$$


Claim 4.5. If $\rho_{j, n} \ll \tilde{\rho}_{n}$ then

$$
\lim _{n \rightarrow \infty}\left[\left\|\chi\left(x / \tilde{\rho}_{n}\right) U_{0, n}^{j}\right\|_{\dot{H}^{1}}+\left\|\chi\left(x / \tilde{\rho}_{n}\right) U_{1, n}^{j}\right\|_{L^{2}}\right]=0 .
$$

If $\tilde{\rho}_{n} \ll \rho_{j, n}$ then

$$
\lim _{n \rightarrow \infty}\left[\left\|\chi\left(x / \tilde{\rho}_{n}\right) U_{0, n}^{j}-U_{0, n}^{j}\right\|_{\dot{H}^{1}}+\left\|\chi\left(x / \tilde{\rho}_{n}\right) U_{1, n}^{j}-U_{1, n}^{j}\right\|_{L^{2}}\right]=0 .
$$

Proof. Indeed by Lemma 4.1 .

$$
\lim _{R \rightarrow \infty} \liminf _{n \rightarrow \infty} \int_{\rho_{j, n} / R \leq|x| \leq R \rho_{j, n}}\left[\left|\nabla U_{0, n}^{j}\right|^{2}+\left|U_{1, n}^{j}\right|^{2}\right]=\int_{\mathbb{R}^{N}}\left[\left|\nabla U_{0, n}^{j}\right|^{2}+\left|U_{1, n}^{j}\right|^{2}\right] .
$$

In the case $\rho_{j, n} \ll \tilde{\rho}_{n}$, choose $\varepsilon>0$ and $R=R(\varepsilon)$ such that

$$
\limsup _{n \rightarrow \infty} \int_{R \rho_{j, n} \leq|x|}\left[\left|\nabla U_{0, n}^{j}\right|^{2}+\left|U_{1, n}^{j}\right|^{2}\right] \leq \varepsilon
$$

As $R \rho_{j, n} \ll \tilde{\rho}_{n}$, we see that for large $n$,

$$
\left\|\chi\left(x / \tilde{\rho}_{n}\right) U_{0, n}^{j}\right\|_{\dot{H}^{1}}^{2}+\left\|\chi\left(x / \tilde{\rho}_{n}\right) U_{1, n}^{j}\right\|_{L^{2}}^{2} \leq \int_{R \rho_{j, n} \leq|x|}\left[\left|\nabla U_{0, n}^{j}\right|^{2}+\left|U_{1, n}^{j}\right|^{2}\right] \leq \varepsilon,
$$

which shows the first estimate of the claim. The proof of the second one is similar and we skip it.

Let us denote by $\mathcal{J}_{\text {ext }}$ the set of indices $j$ such that $\tilde{\rho}_{n} \ll \rho_{j, n}$. Note that for $j \in \mathcal{J}_{\text {ext }}$ we have $j>J_{0}$ and thus

$$
\begin{aligned}
j \in \mathcal{J}_{\mathrm{ext}} \Rightarrow & \left(E\left(U_{0}^{j}, U_{1}^{j}\right) \leq\left(\delta_{1}\left(C_{0}\right)\right)^{2} / N \text { and }\left\|\nabla U_{0}^{j}\right\|_{L^{2}}<\|\nabla W\|_{L^{2}}\right) \\
& \text { or }\left(\lim _{n \rightarrow \infty}-t_{j, n} / \lambda_{j, n}= \pm \infty \text { and } E\left(U_{0}^{j}, U_{1}^{j}\right)<E(W, 0)\right),
\end{aligned}
$$

so the corresponding nonlinear profile $U^{j}$ is globally defined and scatters in both time directions. In view of Claim 4.5, we rewrite (4.19), (4.20) as

$$
\begin{gathered}
\chi\left(x / \tilde{\rho}_{n}\right) u\left(\tau_{n}, x\right)=v\left(\tau_{n}, x\right)+\sum_{\substack{j \in \mathcal{J}_{\mathrm{ext}} \\
j \leq J}} U_{0, n}^{j}(x)+\tilde{w}_{0, n}^{J}(x), \\
\chi\left(x / \tilde{\rho}_{n}\right) \partial_{t} u\left(\tau_{n}, x\right)=\partial_{t} v\left(\tau_{n}, x\right)+\sum_{\substack{j \in \mathcal{J}_{\mathrm{ext}} \\
j \leq J}} U_{1, n}^{j}(x)+\tilde{w}_{1, n}^{J}(x),
\end{gathered}
$$

where

$$
\tilde{w}_{0, n}^{J}=\chi\left(x / \tilde{\rho}_{n}\right) w_{0, n}^{J}+o_{n}(1) \text { in } \dot{H}^{1}, \quad \tilde{w}_{1, n}^{J}=\chi\left(x / \tilde{\rho}_{n}\right) w_{1, n}^{J}+o_{n}(1) \text { in } L^{2} .
$$

By Claim 2.11.

$$
\lim _{n \rightarrow \infty} \limsup _{J \rightarrow \infty}\left\|\tilde{w}_{n}^{J}\right\|_{S(\mathbb{R})}=0 .
$$


Indeed, if 4.23 does not hold, one can find, in view of 2.12), sequences $\left\{n_{k}\right\}_{k},\left\{J_{k}\right\}_{k}$ and $\varepsilon>0$ such that

$$
\forall k,\left\|\tilde{w}_{n_{k}}^{J_{k}}\right\|_{S(\mathbb{R})} \geq \varepsilon \quad \text { and } \quad \lim _{k \rightarrow \infty}\left\|w_{n_{k}}^{J_{k}}\right\|_{S(\mathbb{R})}=0
$$

a contradiction with Claim 2.11.

By (4.23), the decomposition 4.21, 4.22) is a profile decomposition of the sequence

$$
\chi\left(x / \tilde{\rho}_{n}\right)\left(u\left(\tau_{n}, x\right), \partial_{t} u\left(\tau_{n}, x\right)\right) .
$$

Denote by $\tilde{u}_{n}$ the solution of $(1.1)$ such that

$$
\tilde{u}_{n \uparrow t=\tau_{n}}=\chi\left(x / \tilde{\rho}_{n}\right) u\left(\tau_{n}, x\right), \quad \partial_{t} \tilde{u}_{n \uparrow t=\tau_{n}}=\chi\left(x / \tilde{\rho}_{n}\right) \partial_{t} u\left(\tau_{n}, x\right) .
$$

Using that all the nonlinear solutions $U^{j}, j \in \mathcal{J}_{\text {ext }}$, are globally defined and scatter, we deduce by Proposition 2.8 that $\tilde{u}_{n}$ is globally defined for large $n$ and

$$
\tilde{u}_{n}\left(\tau_{n}+t, x\right)=v\left(\tau_{n}+t, x\right)+\sum_{\substack{j \in \mathcal{J}_{\text {ext }} \\ j \leq J}} U_{n}^{j}(t, x)+\tilde{w}_{n}^{J}(t, x)+r_{n}^{J}(t, x),
$$

where $r_{n}^{J}$ satisfies 2.23). By the definition of $\tilde{u}_{n}$,

$$
\tilde{u}_{n}\left(\tau_{n}, x\right)=u\left(\tau_{n}, x\right), \quad \partial_{t} \tilde{u}_{n}\left(\tau_{n}, x\right)=\partial_{t} u\left(\tau_{n}, x\right) \quad \text { if }|x| \geq 2 \tilde{\rho}_{n} .
$$

By finite speed of propagation, as long as $0 \leq \tau_{n}+t<1$, we have

$$
\tilde{u}_{n}\left(\tau_{n}+t, x\right)=u\left(\tau_{n}+t, x\right), \quad \partial_{t} \tilde{u}_{n}\left(\tau_{n}+t, x\right)=\partial_{t} u\left(\tau_{n}+t, x\right) \quad \text { if }|x| \geq 2 \tilde{\rho}_{n}+|t| .
$$

The key point of the proof is the following claim:

Claim 4.6. The set $\mathcal{J}_{\text {ext }}$ is empty.

Proof. The proof takes several steps.

Step 1: No profile dispersing backward in time. Let $k \in \mathcal{J}_{\text {ext }}$. We first show that we cannot have $-t_{k, n} / \lambda_{k, n} \rightarrow-\infty$. Let us assume that $-t_{k, n} / \lambda_{k, n} \rightarrow-\infty$. Then $\rho_{k, n}=\left|t_{k, n}\right|$. Furthermore $U^{k}$ scatters backward in time. As a consequence, by Lemma 4.1, if $M$ is large enough, there exists $\varepsilon_{k}>0$ such that for all large $n$,

$$
\int_{|x| \geq t_{k, n}+\tau_{n}-M \lambda_{k, n}}\left[\left|\nabla U_{n}^{k}\left(-\tau_{n}, x\right)\right|^{2}+\left|\partial_{t} U_{n}^{k}\left(-\tau_{n}, x\right)\right|^{2}\right] d x \geq \varepsilon_{k} .
$$

As $k \in \mathcal{J}_{\text {ext }}$, we know that $t_{k, n}=\rho_{k, n} \gg \tilde{\rho}_{n}$. Furthermore $\lambda_{k, n}=o\left(\left|t_{k, n}\right|\right)$. Thus for large $n, t_{k, n}+\tau_{n}-M \lambda_{k, n} \gg 2 \tilde{\rho}_{n}+\tau_{n}$, and the preceding inequality implies

$$
\int_{|x| \geq 2 \tilde{\rho}_{n}+\tau_{n}}\left[\left|\nabla U_{n}^{k}\left(-\tau_{n}, x\right)\right|^{2}+\left|\partial_{t} U_{n}^{k}\left(-\tau_{n}, x\right)\right|^{2}\right] d x \geq \varepsilon_{k}
$$


Using again that $U^{k}$ scatters backward in time and that $\tilde{\rho}_{n} \ll t_{k, n}$, by Lemma 4.1 we get

$$
\int_{|x| \leq 2 \tilde{\rho}_{n}+\tau_{n}}\left[\left|\nabla U_{n}^{k}\left(-\tau_{n}, x\right)\right|^{2}+\left|\partial_{t} U_{n}^{k}\left(-\tau_{n}, x\right)\right|^{2}\right] d x=o_{n}(1) .
$$

Let $j \in \mathcal{J}_{\text {ext }} \backslash\{k\}$. Then $U^{j}$ scatters in both time directions, and there exists a solution $V_{\mathrm{L}}^{j}$ of the linear wave equation such that

$$
\lim _{t \rightarrow-\infty}\left[\left\|V_{\mathrm{L}}^{j}(t)-U^{j}(t)\right\|_{\dot{H}^{1}}+\left\|\partial_{t} V_{\mathrm{L}}^{j}(t)-\partial_{t} U^{j}(t)\right\|_{L^{2}}\right]=0 .
$$

Noting $V_{\mathrm{L}, n}^{j}(t, x)=\frac{1}{\lambda_{j, n}^{1 / 2}} V_{\mathrm{L}}^{j}\left(\frac{t-t_{j, n}}{\lambda_{j, n}}, \frac{x}{\lambda_{j, n}}\right)$, we get, by conservation of energy for the linear wave equation and 4.28 ,

$$
\begin{aligned}
\int_{|x| \geq \tau_{n}+2 \tilde{\rho}_{n}} \nabla_{t, x} U_{n}^{j}\left(-\tau_{n}\right) & \cdot \nabla_{t, x} U_{n}^{k}\left(-\tau_{n}\right) d x=\int_{\mathbb{R}^{3}} \nabla_{t, x} U_{n}^{j}\left(-\tau_{n}\right) \cdot \nabla_{t, x} U_{n}^{k}\left(-\tau_{n}\right) d x+o_{n}(1) \\
= & \int_{\mathbb{R}^{3}} \nabla_{t, x} V_{\mathrm{L}, n}^{j}(0) \cdot \nabla_{t, x} U_{\mathrm{L}, n}^{k}(0) d x+o_{n}(1)=o_{n}(1)
\end{aligned}
$$

where we have used the orthogonality of the parameters $\left(\lambda_{j, n}, t_{j, n}\right)$ and $\left(\lambda_{k, n}, t_{k, n}\right)$. Similarly, if $J>k$,

$$
\begin{aligned}
\int_{|x| \geq \tau_{n}+2 \tilde{\rho}_{n}} \nabla_{t, x} U_{n}^{k}\left(-\tau_{n}, x\right) \cdot \nabla_{t, x} \tilde{w}_{n}^{J}\left(-\tau_{n}, x\right) d x \\
\quad=\int \nabla_{t, x} U_{n}^{k}\left(-\tau_{n}, x\right) \cdot \nabla_{t, x} \tilde{w}_{n}^{J}\left(-\tau_{n}, x\right) d x+o_{n}(1) \\
=\int \nabla_{t, x} V_{\mathrm{L}, n}^{k}\left(-\tau_{n}, x\right) \cdot \nabla_{t, x} \tilde{w}_{n}^{J}\left(-\tau_{n}, x\right) d x+o_{n}(1) \\
=\int \nabla_{t, x} V_{\mathrm{L}, n}^{k}\left(-t_{k, n}, x\right) \cdot \nabla_{t, x} \tilde{w}_{n}^{J}(0, x) d x+o_{n}(1)=o_{n}(1) .
\end{aligned}
$$

At the last line, we used the conservation of energy, and the fact that by (4.23), the $\tilde{w}_{n}^{J}$ are the remainders of the profile decomposition 4.21$], 44.22$ ) and thus by 2.13 ,

$$
\lambda_{k, n}^{N / 2} \nabla_{t, x} \tilde{w}_{n}^{J}\left(t_{k, n}, \lambda_{k, n} x\right) \underset{n \rightarrow \infty}{\longrightarrow} 0 \quad \text { in }\left(L^{2}\right)^{N+1} .
$$

Combining 4.24) with $t=-\tau_{n}$, 4.25, 4.27, 4.29) and 4.30) we get, if $n$ is large enough,

$$
\int_{|x| \geq \tau_{n}+2 \tilde{\rho}_{n}}\left[\left|\nabla_{t, x} u(0, x)\right|^{2}-\left|\nabla_{t, x} v(0, x)\right|^{2}\right] \geq \frac{\varepsilon_{k}}{2} .
$$

As the function $x \mapsto\left|\nabla_{t, x} u(0, x)\right|^{2}-\left|\nabla_{t, x} v(0, x)\right|^{2}$ is supported in $\{|x| \leq 1\}$, we get

$$
\left.\int_{2 \tilde{\rho}_{n}+\left|\tau_{n}\right| \leq|x| \leq 1}|| \nabla_{t, x} u(0, x)\right|^{2}-\left|\nabla_{t, x} v(0, x)\right|^{2} \mid \geq \frac{\varepsilon_{k}}{2} .
$$

Letting $n \rightarrow \infty$ we have $2 \tilde{\rho}_{n}+\left|\tau_{n}\right| \rightarrow 1$, which yields a contradiction. 
Step 2: No profile dispersing forward in time. We next show by contradiction that if $k \in \mathcal{J}_{\text {ext }}$ we cannot have $\lim _{n \rightarrow \infty}-t_{k, n} / \lambda_{k, n}=\infty$. Let $\sigma_{n}=\left(1-\tau_{n}\right) / 2$. Using that $U^{k}$ scatters forward in time, Lemma 4.1 implies that if $M$ is large enough, there exists $\varepsilon_{k}>0$ such that for all large $n$,

$$
\int_{|x| \geq\left|t_{k, n}\right|+\sigma_{n}-M \lambda_{k, n}}\left|\nabla_{t, x} U_{n}^{k}\left(\sigma_{n}, x\right)\right|^{2} d x \geq \varepsilon_{k} .
$$

By Lemma 4.1, we also have (using that $\lambda_{k, n} \ll\left|t_{k, n}\right|$ )

$$
\lim _{n \rightarrow \infty} \int_{|x| \leq \sigma_{n}}\left|\nabla_{t, x} U_{n}^{k}\left(\sigma_{n}, x\right)\right|^{2} d x=0,
$$

from which we can deduce the analogues of the ortogonality conditions 4.29) and 4.30) with $\tau_{n}+2 \tilde{\rho}_{n}$ replaced by $\sigma_{n}$. As in the preceding case, using (4.24) with $t=\sigma_{n}$ we deduce from 4.31, that for large $n$,

$$
\int_{|x| \geq \sigma_{n}}\left[\left|\nabla_{t, x} u\left(\frac{1+\tau_{n}}{2}, x\right)\right|^{2}-\left|\nabla_{t, x} v\left(\frac{1+\tau_{n}}{2}, x\right)\right|^{2}\right] \geq \frac{\varepsilon_{k}}{2} .
$$

As $1-\left(1+\tau_{n}\right) / 2=\sigma_{n}$, this contradicts the fact that $|x| \leq 1-t$ on the support of $u-v$.

Step 3: No compact profile. In this step we conclude the proof, showing that $\mathcal{J}_{\text {ext }}$ is empty. According to Steps 1 and 2 , for all $j \in \mathcal{J}_{\text {ext }}$ and all $n, t_{j, n}=0$, and we can rewrite 4.24) as

$$
\tilde{u}_{n}\left(\tau_{n}+t\right)=v\left(\tau_{n}+t, x\right)+\sum_{\substack{j \in \mathcal{J}_{\text {ext }} \\ j \leq J}} \frac{1}{\lambda_{j, n}^{1 / 2}} U^{j}\left(\frac{t}{\lambda_{j, n}}, \frac{x}{\lambda_{j, n}}\right)+\tilde{w}_{n}^{J}(t, x)+r_{n}^{J}(t, x),
$$

Furthermore, we know that $j>J_{0}$ for $j \in \mathcal{J}_{\text {ext }}$, and thus by the definition of $J_{0}$ we have

$$
\sqrt{\left\|\nabla U_{0}^{j}\right\|^{2}+\left\|U_{1}^{j}\right\|^{2}} \leq \delta_{1}\left(C_{0}\right)
$$

Assume that $\mathcal{J}_{\text {ext }}$ is not empty. Then by assumption (4.1) for large $J$,

$$
0<\eta^{2}=\sum_{\substack{j \in \mathcal{J}_{\text {ext }} \\ j \leq J}}\left[\left\|\nabla U_{0}^{j}\right\|_{L^{2}}^{2}+\left\|U_{1}^{j}\right\|_{L^{2}}^{2}\right] \leq C_{0}^{2} .
$$

Choose $J$ such that

$$
\sup _{t \in \mathbb{R}} \sqrt{\left\|\nabla r_{n}^{J}(t)\right\|_{L^{2}}^{2}+\left\|\partial_{t} r_{n}^{J}(t)\right\|_{L^{2}}} \leq \frac{\eta}{8}
$$

Let $k \in \mathcal{J}_{\text {ext }}$ be such that $k \leq J$ and

$$
\lambda_{k}=\inf _{\substack{j \in \mathcal{J}_{\mathrm{ext}} \\ j=1, \ldots, J}} \lambda_{j}
$$


By 4.33, we can use Corollary 4.3, which implies that there exists $r_{0}>0$ such that the following occurs for all $t \in\left[-\tau_{n}, 0\right)$ or for all $t \in\left(0,1-\tau_{n}\right)$ :

$$
\int_{|x| \geq \lambda_{k, n} r_{0}+|t|}\left[\left|\nabla U_{n}(t, x)\right|^{2}+\left|\partial_{t} U_{n}(t, x)\right|^{2}\right] d x \geq \frac{\eta^{2}}{16},
$$

where

$$
U_{n}=\sum_{j \in \mathcal{J}_{\mathrm{ext}}} \frac{1}{\lambda_{j, n}^{1 / 2}} U^{j}\left(\frac{t}{\lambda_{j, n}}, \frac{x}{\lambda_{j, n}}\right)+\tilde{w}_{n}^{J}(t, x) .
$$

And thus by (4.34), for all $t \in\left[-\tau_{n}, 0\right)$ or for all $t \in\left(0,1-\tau_{n}\right)$,

$$
\int_{|x| \geq \lambda_{k, n} r_{0}+|t|}\left[\left|\nabla\left(\tilde{u}_{n}\left(\tau_{n}+t\right)-v\left(\tau_{n}+t\right)\right)\right|^{2}+\left|\partial_{t} \tilde{u}_{n}\left(\tau_{n}+t\right)-\partial_{t} v\left(\tau_{n}+t\right)\right|^{2}\right] \geq \frac{\eta^{2}}{64} .
$$

First assume that this holds for all $t \in\left(0,1-\tau_{n}\right)$. Letting $t_{n}=\left(1-\tau_{n}-r_{0} \lambda_{k, n}\right) / 2$ in 4.35, we obtain, for large $n$,

$$
\int_{|x| \geq 1-\tau_{n}-t_{n}}\left[\left|\nabla\left(\tilde{u}_{n}\left(\tau_{n}+t_{n}\right)-v\left(\tau_{n}+t_{n}\right)\right)\right|^{2}+\left|\partial_{t} \tilde{u}_{n}\left(\tau_{n}+t_{n}\right)-\partial_{t} v\left(\tau_{n}+t_{n}\right)\right|^{2}\right] \geq \frac{\eta^{2}}{64} .
$$

Furthermore, as $k \in \mathcal{J}_{\text {ext }}$, we have $2 \tilde{\rho}_{n}+t_{n} \leq 1-\tau_{n}-t_{n}$, and thus by 4.26, $|x| \leq$ $1-\tau_{n}-t_{n}$ on the support of $u\left(\tau_{n}+t_{n}, \cdot\right)-v\left(\tau_{n}+t_{n}, \cdot\right)$, a contradiction.

It remains to treat the case when (4.35) holds for all $t \in\left[-\tau_{n}, 0\right)$. Then (4.35) with $t=-\tau_{n}$ yields

$$
\left.\left.\int_{|x| \geq \lambda_{k, n} r_{0}+\tau_{n}}\left[\left|\nabla\left(\tilde{u}_{n}(0)-v(0)\right)\right|^{2}+\mid \partial_{t} \tilde{u}_{n}(0)-\partial_{t} v(0)\right)\right|^{2}\right] \geq \frac{\eta^{2}}{64},
$$

which is again a contradiction, recalling that $\left(\tilde{u}_{n}(0, x), \partial_{t} \tilde{u}_{n}(0, x)\right)$ and $\left(u(0, x), \partial_{t} u(0, x)\right)$ coincide for $|x| \geq \tau_{n}+2 \tilde{\rho}_{n}$, and thus for $|x| \geq \tau_{n}+\lambda_{k, n} r_{0}$ for large $n$. The proof of Claim 4.6 is complete.

To finish the proof of Proposition 4.4, we must show that if $\bar{\rho}_{n}$ is as in (4.16), and $J$ is large, then

$$
\lim _{n \rightarrow \infty} \int_{|x| \geq \bar{\rho}_{n}}\left[\left|\nabla w_{0, n}^{J}\right|^{2}+\left(w_{1, n}^{J}\right)^{2}\right] d x=0 .
$$

We will use that $w_{n}^{J}$ is a radial solution of the linear wave equation 2.1. By (4.8),

$$
\int_{\bar{\rho}_{n}}^{\infty}\left(\partial_{r}\left(r w_{0, n}^{J}\right)\right)^{2} d r=\int_{|x| \geq \bar{\rho}_{n}}\left|\nabla w_{n}^{J}\right|^{2} d x-\bar{\rho}_{n} w_{0, n}^{J}\left(\bar{\rho}_{n}\right)^{2} .
$$

By the construction of the profile decomposition (see 2.13), we can choose $J$ so large that

$$
\bar{\rho}_{n}^{1 / 2} w_{0, n}^{J}\left(\bar{\rho}_{n} \cdot\right) \underset{n \rightarrow \infty}{\longrightarrow} 0 \quad \text { in } \dot{H}^{1} .
$$


The map $u \mapsto u(1)$ is a continuous linear form on the vector space of radial functions in $\dot{H}^{1}$. Thus 4.38 implies

$$
\lim _{n \rightarrow \infty} \bar{\rho}_{n}^{1 / 2} w_{0, n}^{J}\left(\bar{\rho}_{n}\right)=0 .
$$

To show (4.36), assume for contradiction that after extraction (in $n$ ), for large $n$,

$$
\int_{|x| \geq \bar{\rho}_{n}}\left[\left|\nabla w_{0, n}^{J}\right|^{2}+\left(w_{1, n}^{J}\right)^{2}\right] d x \geq \varepsilon_{0} .
$$

Then by 4.37) and 4.39), for large $n$,

$$
\int_{\bar{\rho}_{n}}^{\infty}\left[\left(\partial_{r}\left(r w_{0, n}^{J}\right)\right)^{2}+\left(r w_{1, n}^{J}\right)^{2}\right] d r \geq \frac{\varepsilon_{0}}{2} .
$$

By Lemma 4.2 and still extracting subsequences, the following holds for all $t>0$ or all $t<0$, and for all large $n$ :

$$
\int_{\bar{\rho}_{n}+|t|}^{\infty}\left[\left(\partial_{r}\left(r w_{n}^{J}(t)\right)\right)^{2}+\left(r \partial_{t} w_{n}^{J}(t)\right)^{2}\right] d r \geq \frac{\varepsilon_{0}}{4} .
$$

By [4.8), this implies that for all $t>0$ or for all $t<0$,

$$
\int_{|x| \geq \bar{\rho}_{n}+|t|}\left[\left|\nabla w_{n}^{J}(t)\right|^{2}+\left(\partial_{t} w_{n}^{J}(t)\right)^{2}\right] d x \geq \frac{\varepsilon_{0}}{4} .
$$

By finite speed of propagation, we have

$$
\tilde{w}_{n}^{J}(t, x)=w_{n}^{J}(t, x), \quad|x| \geq 2 \tilde{\rho}_{n}+|t| .
$$

As $\tilde{\rho}_{n} \ll \bar{\rho}_{n}$, we obtain for large $n$,

$$
\int_{|x| \geq \bar{\rho}_{n}+|t|}\left[\left|\nabla \tilde{w}_{n}^{J}(t)\right|^{2}+\left(\partial_{t} \tilde{w}_{n}^{J}(t)\right)^{2}\right] d x \geq \frac{\varepsilon_{0}}{4},
$$

for all $t \geq 0$ or for all $t \leq 0$. In view of Claim 4.6 , the equality 4.32 can be rewritten

$$
\tilde{u}_{n}\left(\tau_{n}+t\right)=v\left(\tau_{n}+t, x\right)+\tilde{w}_{n}^{J}(t, x)+r_{n}^{J}(t, x), \quad 0 \leq \tau_{n}+t<1 .
$$

Taking $t=-\tau_{n}$ if 4.41 holds for all $t<0$, and $t=\left(1-\tau_{n}\right) / 2$ if 4.41 holds for all $t>0$, we get a contradiction as in the proof of Claim 4.6 , concluding the proof of Proposition 4.4 


\section{Rigidity argument for non-self-similar blow-up}

In the section we consider, as in the preceding one, a radial solution in space dimension $N=3$ that blows up at time $T=1$ and satisfies 4.1 . We assume in addition that there exist sequences $\left\{\tau_{n}\right\},\left\{\lambda_{n}\right\}$ such that $\tau_{n} \in(0,1), \tau_{n} \rightarrow 1$ and

$$
\begin{gathered}
\lambda_{n} \ll 1-\tau_{n}, \\
\lim _{n \rightarrow \infty} \int_{|x| \geq \lambda_{n}}\left[\left|\nabla a\left(\tau_{n}\right)\right|^{2}+\left(\partial_{t} a\left(\tau_{n}\right)\right)^{2}+\frac{1}{|x|^{2}}\left(a\left(\tau_{n}\right)\right)^{2}\right]=0,
\end{gathered}
$$

where $a=u-v$ is as usual the singular part of $u$. The main result of this section is the following

Proposition 5.1. Assume that $u$ is radial and that (4.1), (5.1) and 5.2) hold. Then there exist a sequence $\left\{t_{n}\right\}, J_{0}>0,\left\{\iota_{j}\right\}_{j=1}^{J_{0}} \in\{ \pm 1\}^{J_{0}}$, and, for $j=1, \ldots, J_{0}$, sequences $\left\{\lambda_{j, n}\right\}_{n}$ of positive numbers, such that

$$
\begin{aligned}
& u\left(t_{n}, x\right)=v\left(t_{n}, x\right)+\sum_{j=1}^{J_{0}} \frac{\iota_{j}}{\lambda_{j, n}^{1 / 2}} W\left(\frac{x}{\lambda_{j, n}}\right)+w_{0, n} \quad \text { in } \dot{H}^{1}, \\
& \partial_{t} u\left(t_{n}, x\right)=\partial_{t} v\left(t_{n}, x\right)+o_{n}(1) \quad \text { in } L^{2} \text {, }
\end{aligned}
$$

where, denoting by $w_{n}$ the solution of 2.1 with initial data $\left(w_{0, n}, 0\right)$,

$$
\lim _{n \rightarrow \infty}\left\|w_{n}\right\|_{S(\mathbb{R})}=0
$$

Let us mention that the assumption $N=3$ is not essential for the arguments of this section. In $\$ 5.1$ we show that assumptions 5.1, (5.2) imply that $\partial_{t} a$ is small in $L^{2}$ for a sequence of times. Proposition 5.1 is proven in $\$ 5.2$

\subsection{Smallness of the time derivative of the solution}

Lemma 5.2. Assume (5.2). Then

$$
\lim _{n \rightarrow \infty} \frac{1}{1-\tau_{n}} \int_{\tau_{n}}^{1} \int_{\mathbb{R}^{3}}\left(\partial_{t} a\right)^{2} d x d t=0 .
$$

Corollary 5.3. Under the assumption of Lemma 5.2 there exists an increasing sequence $t_{n} \rightarrow 1$ with $t_{n} \in(0,1)$ such that

$$
\begin{gathered}
\forall n, \quad \int_{\mathbb{R}^{3}}\left|\partial_{t} a\left(t_{n}, x\right)\right|^{2} d x \leq \frac{1}{n}, \\
\forall n, \forall \sigma \in\left(0,1-t_{n}\right), \quad \frac{1}{\sigma} \int_{t_{n}}^{t_{n}+\sigma} \int_{\mathbb{R}^{3}}\left|\partial_{t} a(t, x)\right|^{2} d x d t \leq \frac{1}{n} .
\end{gathered}
$$


Let us first assume Lemma 5.2 and prove Corollary 5.3 .

Proof of Corollary 5.3 Using that the map $t \mapsto \partial_{t} a(t, \cdot)$ is continuous from $(0,1)$ to $L^{2}\left(\mathbb{R}^{3}\right)$ we get 5.5 from 5.6 by letting $\sigma \rightarrow 0$.

The existence of a sequence $\left\{t_{n}\right\}$ satisfying (5.6) is equivalent to

$\forall \varepsilon>0, \forall t_{*} \in(0,1), \exists t_{0} \in\left(t_{*}, 1\right), \forall \sigma \in\left(0,1-t_{0}\right), \quad \frac{1}{\sigma} \int_{t_{0}}^{t_{0}+\sigma} \int_{\mathbb{R}^{3}}\left(\partial_{t} a\right)^{2} d x d t \leq \varepsilon$.

Assume for contradiction that

$\exists \varepsilon>0, \exists t_{*} \in(0,1), \forall t_{0} \in\left(t_{*}, 1\right), \exists \sigma \in\left(0,1-t_{0}\right), \quad \frac{1}{\sigma} \int_{t_{0}}^{t_{0}+\sigma} \int_{\mathbb{R}^{3}}\left(\partial_{t} a\right)^{2} d x d t>\varepsilon$.

By Lemma 5.2 we can fix a large $n$ such that $\tau_{n}>t_{*}$ and

$$
\frac{1}{1-\tau_{n}} \int_{\tau_{n}}^{1} \int_{\mathbb{R}^{3}}\left(\partial_{t} a\right)^{2} d x d t \leq \frac{\varepsilon}{2}
$$

Let

$$
A=\left\{\sigma \in\left(0,1-\tau_{n}\right): \frac{1}{\sigma} \int_{\tau_{n}}^{\tau_{n}+\sigma} \int_{\mathbb{R}^{3}}\left(\partial_{t} a\right)^{2} d x d t \geq \varepsilon\right\} .
$$

By (5.7), $A$ is not empty. Furthermore, it is closed in $\left(0,1-\tau_{n}\right)$. Let $\theta_{0}=\sup A$. By the choice of $n, \theta_{0} \neq 1-\tau_{n}$. Furthermore,

$$
\int_{\tau_{n}}^{\tau_{n}+\theta_{0}} \int_{\mathbb{R}^{3}}\left(\partial_{t} a\right)^{2} d x d t \geq \varepsilon \theta_{0}
$$

By (5.7), using that $t_{*}<\tau_{n}+\theta_{0}<1$, there exists $\sigma \in\left(0,1-\tau_{n}-\theta_{0}\right)$ such that

$$
\int_{\tau_{n}+\theta_{0}}^{\tau_{n}+\theta_{0}+\sigma} \int_{\mathbb{R}^{3}}\left(\partial_{t} a\right)^{2} d x d t>\varepsilon \sigma .
$$

Summing up the preceding two inequalities, we get

$$
\int_{\tau_{n}}^{\tau_{n}+\theta_{0}+\sigma} \int_{\mathbb{R}^{3}}\left(\partial_{t} a\right)^{2} d x d t>\varepsilon\left(\theta_{0}+\sigma\right)
$$

with $\theta_{0}+\sigma \in\left(\theta_{0}, 1-\tau_{n}\right)$. Thus $\theta_{0}+\sigma \in A$ and $\theta_{0}+\sigma>\theta_{0}$, contradicting the fact that $\theta_{0}=\sup A$.

It remains to prove Lemma 5.2

Proof of Lemma 5.2. Let

$$
z_{1}(t)=\int_{\mathbb{R}^{3}}\left[\left(u \partial_{t} u\right)-\left(v \partial_{t} v\right)\right] d x, \quad z_{2}(t)=\int_{\mathbb{R}^{3}}\left[x \cdot \nabla u \partial_{t} u-x \cdot \nabla v \partial_{t} v\right] d x .
$$


As $u-v$ and $\partial_{t}(u-v)$ are compactly supported in the space variable, both integrals are well-defined. We first show

$$
\lim _{n \rightarrow \infty} \frac{\left|z_{1}\left(\tau_{n}\right)\right|+\left|z_{2}\left(\tau_{n}\right)\right|}{1-\tau_{n}}=0 .
$$

Indeed, write

$$
z_{1}\left(\tau_{n}\right)=\int_{\mathbb{R}^{3}} a\left(\tau_{n}\right) \partial_{t} u\left(\tau_{n}\right)+\int_{\mathbb{R}^{3}} v\left(\tau_{n}\right) \partial_{t} a\left(\tau_{n}\right) .
$$

Then, using the fact that $|x| \leq 1-\tau_{n}$ on the supports of $a\left(\tau_{n}\right)$ and $\partial_{t} a\left(\tau_{n}\right)$,

$$
\begin{aligned}
\left|\int a\left(\tau_{n}\right) \partial_{t} u\left(\tau_{n}\right)\right| & \leq \int_{|x| \leq \lambda_{n}}\left|a\left(\tau_{n}\right) \partial_{t} u\left(\tau_{n}\right)\right|+\int_{|x| \geq \lambda_{n}}\left|a\left(\tau_{n}\right) \partial_{t} u\left(\tau_{n}\right)\right| \\
& \leq \lambda_{n} \int_{|x| \leq \lambda_{n}} \frac{1}{|x|}\left|a\left(\tau_{n}\right) \partial_{t} u\left(\tau_{n}\right)\right|+\left(1-\tau_{n}\right) \int_{|x| \geq \lambda_{n}} \frac{1}{|x|}\left|a\left(\tau_{n}\right) \partial_{t} u\left(\tau_{n}\right)\right| .
\end{aligned}
$$

By (5.1) and 5.2),

$$
\left|\int a\left(\tau_{n}\right) \partial_{t} u\left(\tau_{n}\right)\right|=o\left(1-\tau_{n}\right) \quad \text { as } n \rightarrow \infty .
$$

Estimating the other terms in the same way we get 5.8.

Differentiating the definitions of $z_{1}$ and $z_{2}$ and using that both $u$ and $v$ are solutions of (1.1), we get

$$
\begin{aligned}
z_{1}^{\prime}(t)= & \int\left(\partial_{t} u\right)^{2}-\int|\nabla u|^{2}+\int u^{6}-\left[\int\left(\partial_{t} v\right)^{2} d x-\int|\nabla v|^{2} d x+\int v^{6}\right] \\
z_{2}^{\prime}(t)= & -\frac{3}{2} \int\left(\partial_{t} u\right)^{2}+\frac{1}{2}\left(\int|\nabla u|^{2}-\int u^{6}\right) \\
& -\left[-\frac{3}{2} \int\left(\partial_{t} v\right)^{2}+\frac{1}{2}\left(\int|\nabla v|^{2}-\int v^{6}\right)\right]
\end{aligned}
$$

Noting that $|x| \leq 1-t$ on the support of $a$, that $v$ converges in $\dot{H}^{1} \times L^{2}$ as $t \rightarrow 1$, and that $u$ is bounded in $\dot{H}^{1} \times L^{2}$, we get, as $t \rightarrow 1^{-}$,

$$
\begin{aligned}
& z_{1}^{\prime}(t)=\int\left(\partial_{t} a\right)^{2} d x-\int|\nabla a|^{2} d x+\int a^{6} d x+o(1), \\
& z_{2}^{\prime}(t)=-\frac{3}{2} \int\left(\partial_{t} a\right)^{2}+\frac{1}{2}\left(\int|\nabla a|^{2}-\int a^{6}\right)+o(1) .
\end{aligned}
$$

Let

$$
Z(t)=\frac{1}{2} z_{1}(t)+z_{2}(t)
$$

Then

$$
Z^{\prime}(t)=-\int\left(\partial_{t} a\right)^{2}+o(1) \quad \text { as } t \rightarrow 1
$$


Let $\varepsilon>0$, and $m, n$ be two large integers with $n<m$. Integrating the preceding inequality we get

$$
\int_{\tau_{n}}^{\tau_{m}} \int_{\mathbb{R}^{3}}\left(\partial_{t} a\right)^{2} \leq\left|Z\left(\tau_{m}\right)-Z\left(\tau_{n}\right)\right|+\varepsilon\left|\tau_{n}-\tau_{m}\right| .
$$

Letting $m$ tend to infinity we obtain

$$
\int_{\tau_{n}}^{1} \int_{\mathbb{R}^{3}}\left(\partial_{t} a\right)^{2} \leq\left|Z\left(\tau_{n}\right)\right|+o\left(1-\tau_{n}\right) \quad \text { as } n \rightarrow \infty .
$$

From 5.8 we deduce

$$
\int_{\tau_{n}}^{1} \int_{\mathbb{R}^{3}}\left(\partial_{t} a\right)^{2}=o\left(1-\tau_{n}\right) \quad \text { as } n \rightarrow \infty .
$$

\subsection{Decomposition into a sum of rescaled stationary solutions}

The proof of Proposition 5.1 is divided into four steps.

Step 1: Extraction of a sequence and profile decomposition. Extracting a subsequence from $\left\{t_{n}\right\}$, we assume that $\left\{\left(a\left(t_{n}\right), \partial_{t} a\left(t_{n}\right)\right)\right\}_{n}$ admits a profile decomposition with profiles $\left\{U^{j}\right\}$ and parameters $\left\{\lambda_{j, n}, t_{j, n}\right\}$. By the Pythagorean expansion

$$
\left\|\partial_{t} a\left(t_{n}\right)\right\|_{L^{2}}^{2}=\sum_{j=1}^{J}\left\|\partial_{t} U^{j}\left(-t_{j, n} / \lambda_{j, n}\right)\right\|_{L^{2}}^{2}+\left\|w_{n}^{J}\right\|_{L^{2}}^{2}+o(1) \quad \text { as } n \rightarrow \infty
$$

and using 5.5 , we find that for all $j$ (here $U_{n}^{j}$ is the rescaled profiled, defined in Notation 2.77,

$$
\lim _{n \rightarrow \infty}\left\|\partial_{t} U_{n}^{j}(0)\right\|_{L^{2}}=\lim _{n \rightarrow \infty}\left\|\partial_{t} U^{j}\left(-t_{j, n} / \lambda_{j, n}\right)\right\|_{L^{2}}=0 .
$$

We deduce that for all $j$ such that $U^{j} \neq 0,\left\{-t_{j, n} / \lambda_{j, n}\right\}_{n}$ is bounded. Indeed, assume that there exists a subsequence in $n$ such that $-t_{j, n} / \lambda_{j, n} \rightarrow \pm \infty$. Then by definition of $U^{j}$ and the equipartition of energy for solutions of the linear equation 2.1) as $t \rightarrow \pm \infty$,

$$
\frac{1}{2}\left\|U_{\mathrm{L}}^{j}(0)\right\|_{\dot{H}^{1}}^{2}+\frac{1}{2}\left\|\partial_{t} U_{\mathrm{L}}^{j}(0)\right\|_{L^{2}}^{2}=\lim _{n \rightarrow \infty}\left\|\partial_{t} U_{\mathrm{L}}^{j}\left(-t_{j, n} / \lambda_{j, n}\right)\right\|_{L^{2}}^{2}=0,
$$

showing that $U^{j}=0$, a contradiction.

Translating in time the profiles, we may assume

$$
\forall j, \forall n, \quad t_{j, n}=0 .
$$

As a consequence of (5.9), $U_{1}^{j}:=\partial_{t} U^{j}(0)=0$ for all $j$. Let $\delta_{0}>0$ be a small parameter (given by the small data theory for (1.1)). There exists a finite number $J_{0}$ of profiles $U^{j}$ such that $\left\|U_{0}^{j}\right\|_{\dot{H}^{1}}+\left\|U_{1}^{j}\right\|_{L^{2}}=\left\|U_{0}^{j}\right\|_{\dot{H}^{1}} \geq \delta_{0}$. Reordering the profiles, we may assume

$$
\left\|U_{0}^{j}\right\|_{\dot{H}^{1}} \geq \delta_{0} \Leftrightarrow 1 \leq j \leq J_{0} .
$$


In view of 5.10 and the orthogonality of the profiles, we obtain, after a new extraction in $n$,

$$
\forall j, k, \quad j \neq k \Rightarrow \lambda_{j, n} \ll \lambda_{k, n} \text { or } \lambda_{k, n} \ll \lambda_{j, n} .
$$

Thus we may reorder the first profiles so that

$$
\lambda_{J_{0}, n} \ll \lambda_{J_{0}-1, n} \ll \cdots \ll \lambda_{1, n} .
$$

We show that $U^{j} \in\{W,-W\}$ if $1 \leq j \leq J_{0}$ and $U^{j}=0$ if $j>J_{0}$. This is equivalent to the fact that the set of indices $j$ such that $U^{j} \notin\{0, W,-W\}$ is empty. Assume that this set is not empty and let

$$
k_{0}=\min \left\{j \geq 1: U^{j} \notin\{0, W,-W\}\right\} .
$$

Let $k_{1}=\min \left\{1 \leq j \leq J_{0}: \lambda_{j, n} \ll \lambda_{k_{0}, n}\right\}$. If this set is empty, $k_{1}$ is not defined, and we will make the convention $\lambda_{k_{1}, n}=0$. By Claim B.1, there exists a sequence $\tilde{\lambda}_{n} \rightarrow 0$ such that

$$
\begin{aligned}
\lambda_{k_{1}, n} & \ll \tilde{\lambda}_{n} \ll \lambda_{k_{0}, n}, \\
\forall j, \quad \tilde{\lambda}_{n} & \ll \lambda_{j, n} \text { or } \lambda_{j, n} \ll \tilde{\lambda}_{n} .
\end{aligned}
$$

Let

$$
\mathcal{J}_{\text {ext }}=\left\{j \geq 1: \tilde{\lambda}_{n} \ll \lambda_{j, n}\right\} .
$$

Note that by the first inequality in 5.11 ,

$$
\forall j, \quad\left(j \in \mathcal{J}_{\mathrm{ext}} \text { and } \lambda_{j, n} \ll \lambda_{k_{0}, n}\right) \Rightarrow j>J_{0} .
$$

Step 2. Let $T>0$ be in the domain of existence of $U^{k_{0}}$. Using that $\lambda_{k_{0}, n} \lesssim 1-\tau_{n}$, we can choose $T$ small enough so that $\lambda_{k_{0}, n} T<1-t_{n}$ for large $n$. In this step we show

$$
\frac{1}{\lambda_{k_{0}, n} T} \int_{0}^{\lambda_{k_{0}, n} T} \int_{|x| \geq \lambda_{k_{0}, n} \varepsilon+|t|}\left|\partial_{t} U_{n}^{k_{0}}(t, x)+\partial_{t} w_{n}^{J}(t, x)\right|^{2} d x d t=o_{n}^{J},
$$

where by definition $\lim _{J \rightarrow \infty} \lim \sup _{n \rightarrow \infty} o_{n}^{J}=0$. More precisely, we will show the following two estimates which directly imply (5.14):

$$
\begin{gathered}
\frac{1}{\lambda_{k_{0}, n} T} \int_{0}^{\lambda_{k_{0}, n} T} \int_{|x| \geq \lambda_{k_{0}, n} \varepsilon+|t|}\left|\sum_{\substack{j \in \mathcal{J}_{\text {ext }} \\
j \leq J}} \partial_{t} U_{n}^{j}(t, x)+\partial_{t} w_{n}^{J}(t, x)\right|^{2} d x d t=o_{n}^{J}, \\
j \in \mathcal{J}_{\text {ext }} \text { and } j \neq k_{0} \Rightarrow \lim _{n \rightarrow \infty} \frac{1}{\lambda_{k_{0}, n} T} \int_{0}^{\lambda_{k_{0}, n} T} \int_{|x| \geq \varepsilon \lambda_{k_{0}, n}+|t|}\left|\partial_{t} U_{n}^{j}(t, x)\right|^{2} d x d t=0 .
\end{gathered}
$$

Proof of 5.15. Choose a radial function $\chi \in C^{\infty}\left(\mathbb{R}^{3}\right)$ such that $\chi(x)=1$ for $|x| \geq 2$ and $\chi(x)=0$ for $|x| \leq 1$. Let $\tilde{u}_{n}$ be the solution of (1.1) with initial data

$$
\tilde{u}_{n \mid t=t_{n}}=\chi\left(x / \tilde{\lambda}_{n}\right) u\left(t_{n}, x\right), \quad \partial_{t} \tilde{u}_{n \mid t=t_{n}}=\chi\left(x / \tilde{\lambda}_{n}\right) \partial_{t} u\left(t_{n}, x\right) .
$$


Then, by finite speed of propagation, as long as $t_{n}+s$ is in the domain of existence of $u$ and $\tilde{u}_{n}$,

$$
\tilde{u}_{n}\left(t_{n}+s, x\right)=u\left(t_{n}+s, x\right), \quad|x| \geq|s|+2 \tilde{\lambda}_{n} .
$$

Furthermore, letting

$$
\tilde{w}_{0, n}^{J}(x)=\chi\left(x / \tilde{\lambda}_{n}\right) w_{0, n}^{J}(x), \quad \tilde{w}_{1, n}^{J}(x)=\chi\left(x / \tilde{\lambda}_{n}\right) w_{1, n}^{J}(x),
$$

we obtain (recall that $U_{1, n}^{j}=0$ for all $j$ )

$$
\begin{aligned}
\tilde{u}_{n}\left(t_{n}, x\right)-v\left(t_{n}, x\right) & =\sum_{\substack{j \in \mathcal{J}_{\mathrm{ext}} \\
j \leq J}} U_{0, n}^{j}(x)+\tilde{w}_{0, n}^{J}(x)+o_{n}(1) \quad \text { in } \dot{H}^{1}, \\
\partial_{t} \tilde{u}_{n}\left(t_{n}, x\right)-\partial_{t} v\left(t_{n}, x\right) & =\tilde{w}_{1, n}^{J}(x)+o_{n}(1) \quad \text { in } L^{2} .
\end{aligned}
$$

By Claim 2.11 together with the argument that we used to show 4.23,

$$
\lim _{n \rightarrow \infty} \limsup _{J \rightarrow \infty}\left\|\tilde{w}_{n}^{J}\right\|_{S(\mathbb{R})}=0
$$

By 5.19, the two equations 5.17, 5.18) yield a profile decomposition of the sequence

$$
\left\{\left(\tilde{u}\left(t_{n}, x\right)-v\left(t_{n}, x\right), \partial_{t} \tilde{u}\left(t_{n}, x\right)-\partial_{t} v\left(t_{n}, x\right)\right)\right\}_{n} .
$$

The development (5.17), 5.18) satisfies the assumptions of Proposition 2.8 with $\theta_{n}=$ $\lambda_{k_{0}, n} T$. Indeed, for $j>J_{0}$ the solution $U^{j}$ scatters both forward and backward in time. Furthermore by (5.13,

$$
\left(j \in\left\{1, \ldots, J_{0}\right\} \cap \mathcal{J}_{\text {ext }} \text { and } j \neq k_{0}\right) \Rightarrow \lambda_{k_{0}, n} T \ll \lambda_{j, n} .
$$

Thus by Proposition 2.8 , for $s \in\left[0, \lambda_{k_{0}, n} T\right]$,

$$
\tilde{u}_{n}\left(t_{n}+s, x\right)=v\left(t_{n}+s, x\right)+\sum_{\substack{j \in \mathcal{J}_{\text {ext }} \\ j \leq J}} U_{n}^{j}(s, x)+\tilde{w}_{n}^{J}(s, x)+r_{n}^{J}(s, x),
$$

where $r_{n}^{J}$ satisfies 2.23) with $\theta_{n}=\lambda_{k_{0}, n} T$. Let $\varepsilon>0$. We have, for large $n$ (so that $\left.\varepsilon \lambda_{k_{0}, n} \geq 2 \tilde{\lambda}_{n}\right)$

$$
\begin{aligned}
o_{n}(1) & =\frac{1}{\lambda_{k_{0}, n} T} \int_{t_{n}}^{t_{n}+\lambda_{k_{0}, n} T} \int_{\mathbb{R}^{3}}\left|\partial_{t} a(t, x)\right|^{2} d x d t \\
& \geq \frac{1}{\lambda_{k_{0}, n} T} \int_{t_{n}}^{t_{n}+\lambda_{k_{0}, n} T} \int_{|x| \geq \lambda_{k_{0}, n} \varepsilon+|t|}\left|\partial_{t} a(t, x)\right|^{2} d x d t \\
& =\frac{1}{\lambda_{k_{0}, n} T} \int_{t_{n}}^{t_{n}+\lambda_{k_{0}, n} T} \int_{|x| \geq \lambda_{k_{0}, n} \varepsilon+|t|}\left|\left(\partial_{t} u_{n}-\partial_{t} v\right)(t, x)\right|^{2} d x d t,
\end{aligned}
$$

which yields 5.15 in view of 5.20 . 
Proof of 5.16. Let $R_{j, n}=\lambda_{k_{0}, n} / \lambda_{j, n}$. We have

$$
\begin{gathered}
\frac{1}{\lambda_{k_{0}, n} T} \int_{0}^{\lambda_{k_{0}, n} T} \int_{|x| \geq \varepsilon \lambda_{k_{0}, n}+|t|}\left|\partial_{t} U_{n}^{j}(t, x)\right|^{2} d x d t \\
=\frac{1}{T R_{j, n}} \int_{0}^{T R_{j, n}} \int_{|y| \geq \varepsilon R_{j, n}+|s|}\left|\partial_{t} U^{j}(s, y)\right|^{2} d y d s .
\end{gathered}
$$

If $\lambda_{j, n} \ll \lambda_{k_{0}, n}$ (and thus $j>J_{0}$ ), we have $R_{j, n} \rightarrow \infty$. By finite speed of propagation, for all $\eta>0$, there exists $M>0$ such that

$$
\forall s \in \mathbb{R}, \quad \int_{|y| \geq M+|s|}\left|\partial_{t} U^{j}(s, y)\right|^{2} d y \leq \eta,
$$

which implies that the right-hand member of 5.21 tends to 0 as $n \rightarrow \infty$.

If $\lambda_{k_{0}, n} \ll \lambda_{j, n}$, then $R_{j, n} \rightarrow 0$, and thus

$$
\begin{aligned}
\frac{1}{T R_{j, n}} \int_{0}^{T R_{j, n}} \int_{|y| \geq R_{j, n} \varepsilon+|s|}\left|\partial_{t} U^{j}(s, y)\right|^{2} d y d s & \leq \frac{1}{T R_{j, n}} \int_{0}^{T R_{j, n}} \int\left|\partial_{t} U^{j}(s, y)\right|^{2} d y d s \\
& \underset{n \rightarrow \infty}{\longrightarrow} \int\left|\partial_{t} U^{j}(0, y)\right|^{2} d y=0
\end{aligned}
$$

concluding the proof of 5.16.

Step 3: Uniqueness argument and conclusion of the proof. By 5.14,

$$
\frac{1}{T} \int_{0}^{T} \int_{|x| \geq \varepsilon+|t|}\left|\partial_{t} U^{k_{0}}(t, x)+\lambda_{k_{0}, n}^{3 / 2} \partial_{t} w_{n}^{J}\left(\lambda_{k_{0}, n} t, \lambda_{k_{0}, n} x\right)\right|^{2} d x d t=o_{n}^{J}
$$

Consider the mapping $\dot{H}^{1} \times L^{2} \rightarrow \mathbb{R}$,

$$
\left(f_{0}, f_{1}\right) \mapsto \frac{1}{T} \int_{0}^{T} \int_{|x| \geq \varepsilon+|t|} \partial_{t} U^{k_{0}}(t, x) \partial_{t} f(t, x) d x d t
$$

where $f(t, x)$ is the solution of the linear wave equation with initial conditions $\left(f_{0}, f_{1}\right)$. This is a continuous linear form on $\dot{H}^{1} \times L^{2}$. By 2.13,

$$
\left(\lambda_{k_{0}, n}^{1 / 2} w_{0, n}^{J}\left(\lambda_{k_{0}, n} \cdot\right), \lambda_{k_{0}, n}^{3 / 2} w_{1, n}^{J}\left(\lambda_{k_{0}, n} \cdot\right)\right) \underset{n \rightarrow \infty}{\longrightarrow} 0 \quad \text { weakly in } \dot{H}^{1} \times L^{2} .
$$

Hence

$$
\lim _{n \rightarrow \infty} \frac{1}{T} \int_{0}^{T} \int_{|x| \geq \varepsilon+|t|} \partial_{t} U^{k_{0}}(t, x) \lambda_{k_{0}, n}^{3 / 2} \partial_{t} w_{n}^{J}\left(\lambda_{k_{0}, n} t, \lambda_{k_{0}, n} x\right) d x d t=0,
$$

and we conclude that for all $\varepsilon>0$,

$$
\frac{1}{T} \int_{0}^{T} \int_{|x| \geq \varepsilon+|t|}\left|\partial_{t} U^{k_{0}}(t, x)\right|^{2} d x d t=0
$$


Letting $\varepsilon \rightarrow 0$ we get

$$
\frac{1}{T} \int_{0}^{T} \int_{|x| \geq|t|}\left|\partial_{t} U^{k_{0}}(t, x)\right|^{2} d x d t=0 .
$$

This shows that $\partial_{t} U^{k_{0}}(t, x)=0$ if $t \leq|x|$ and $0 \leq t \leq T$. Let

$$
\Omega=\left\{(t, x) \in[0, T] \times \mathbb{R}^{3}:|x| \geq t\right\} .
$$

Then

$$
(t, x) \in \Omega \Rightarrow U^{k_{0}}(t, x)=U_{0}^{k_{0}}(x) .
$$

In $\Omega$, the nonlinear wave equation $\partial_{t}^{2} U^{k_{0}}-\Delta U^{k_{0}}-\left(U^{k_{0}}\right)^{5}=0$ becomes $\Delta U^{k_{0}}=$ $-\left(U^{k_{0}}\right)^{5}$. Thus $U^{k_{0}}$ satisfies in the sense of distributions the elliptic equation

$$
\Delta U_{0}^{k_{0}}=-\left(U_{0}^{k_{0}}\right)^{5} \quad \text { in } \mathbb{R}^{3} \backslash\{0\} .
$$

This shows that $U^{k_{0}}$ is smooth in $\mathbb{R}^{3} \backslash\{0\}$ and satisfies the preceding equation in the classical sense in $\mathbb{R}^{3} \backslash\{0\}$. As a consequence $\Delta U_{0}^{k_{0}}+\left(U_{0}^{k_{0}}\right)^{5}$ is a distribution in $H^{-1}\left(\mathbb{R}^{3}\right)$, supported at the origin. The only distribution with these properties in dimension 3 is 0 and we deduce

$$
\Delta U_{0}^{k_{0}}+\left(U_{0}^{k_{0}}\right)^{5}=0
$$

in the sense of distributions on $\mathbb{R}^{3}$ and thus by Claim 2.2, as $U^{k_{0}}$ is radial,

$$
U^{k_{0}}(x)=\frac{1}{\lambda_{0}^{1 / 2}} W\left(\frac{x}{\lambda_{0}}\right) \quad \text { or } \quad U^{k_{0}}(x)=-\frac{1}{\lambda_{0}^{1 / 2}} W\left(\frac{x}{\lambda_{0}}\right) \quad \text { or } \quad U^{k_{0}}=0,
$$

for some $\lambda_{0}>0$, which yields the desired contradiction. The proof is complete.

\section{All radial compact solutions are stationary}

In this section we show Theorem 2

We will assume without loss of generality that $\lambda$ is continuous on $\left(T_{-}(u), T_{+}(u)\right)$ (see [KM06, Remark 5.4]).

Step 1. We show in this step that the solution is globally defined. Assume that $T_{+}(u)<\infty$. For the sake of simplicity, we will assume that $T_{+}(u)=1$. By a standard argument (see Section 3), $\lambda(t) \leq C(1-t)$. By [KM08, Section 6], self-similar, compact blow-up is excluded, which implies that there exists a sequence $\left\{\tau_{n}\right\}_{n}$ such that

$$
\tau_{n} \in(0,1), \quad \lim _{n \rightarrow \infty} \tau_{n}=1, \quad \lim _{n \rightarrow \infty} \frac{\lambda\left(\tau_{n}\right)}{1-\tau_{n}}=0 .
$$

Using that the regular part of $v$ at the blow-up point $t=1$ is 0 , we find, arguing as in Corollary 5.3. that there exists a sequence $\left\{t_{n}\right\}_{n}$ such that

$$
\forall n, \forall \sigma \in\left(0,1-t_{n}\right), \quad \frac{1}{\sigma} \int_{t_{n}}^{t_{n}+\sigma} \int_{\mathbb{R}^{N}}\left|\partial_{t} u(t, x)\right|^{2} d x d t \leq \frac{1}{n} .
$$


Consider $\left(U_{0}, U_{1}\right) \in \dot{H}^{1} \times L^{2}$ such that for a subsequence,

$$
\lim _{n \rightarrow \infty}\left(\lambda\left(t_{n}\right)^{(N-2) / 2} u\left(t_{n}, \lambda\left(t_{n}\right) x\right), \lambda\left(t_{n}\right)^{N / 2} u\left(t_{n}, \lambda\left(t_{n}\right) x\right)\right)=\left(U_{0}, U_{1}\right) .
$$

Let $U$ be the solution of (1.1) with initial condition $\left(U_{0}, U_{1}\right)$ and $\tau_{0} \in\left(0, T_{+}(U)\right)$. Then by Theorem 2.1 .

$$
\lim _{n \rightarrow \infty} \int_{0}^{\tau_{0}} \int_{\mathbb{R}^{N}} \lambda\left(t_{n}\right)^{N}\left(\partial_{t} u\left(t_{n}+\lambda\left(t_{n}\right) s, \lambda\left(t_{n}\right) x\right)\right)^{2} d x d s=\int_{0}^{\tau_{0}} \int_{\mathbb{R}^{N}}\left(\partial_{t} U(t)\right)^{2} d t .
$$

By 6.1], we obtain

$$
\begin{aligned}
\int_{0}^{\tau_{0}} \int_{\mathbb{R}^{N}} \lambda\left(t_{n}\right)^{N}\left(\partial _ { t } u \left(t_{n}+\right.\right. & \left.\left.\lambda\left(t_{n}\right) s, \lambda\left(t_{n}\right) x\right)\right)^{2} d x d s \\
& =\frac{1}{\lambda\left(t_{n}\right)} \int_{0}^{\tau_{0} \lambda\left(t_{n}\right)} \int_{\mathbb{R}^{N}}\left(\partial_{t} u\left(t_{n}+t, x\right)\right)^{2} d x d t \underset{n \rightarrow \infty}{\longrightarrow} 0 .
\end{aligned}
$$

As a consequence, $\partial_{t} U=0$ for $t \in\left[0, \tau_{0}\right]$. By Claim 2.2. $U=0$ or $U=W$ up to the invariances of the equation. If $U=0$, then $E\left(u_{0}, u_{1}\right)=0$, and as $\left\|u\left(t_{n}\right)\right\|_{\dot{H}^{1}}$ tends to 0 , this implies by Claim 2.3 that $u=0$, contradicting our assumption. Thus $U=W$ up to the invariances, and by conservation of energy we deduce that $E\left(u_{0}, u_{1}\right)=E(W, 0)$.

The solution $u$ of $(1.1)$ has threshold energy $E(W, 0)$, is not globally defined and satisfies $u_{0} \in L^{2}$. By Theorem 2 of [DM08], $N=5$ and $u$ has to be the special solution $W^{+}$constructed in this paper, which satisfies $\|u(t)-W\|_{\dot{H}^{1}} \leq e^{c t}$ as $t \rightarrow-\infty$. This contradicts the fact that $u$ has compact support in space, concluding Step 1.

Step 2. We assume in this step that $\lambda$ is bounded on $[0, \infty)$ or on $(-\infty, 0]$, and show that $E\left(u_{0}, u_{1}\right)=E(W, 0)$. By time symmetry we can assume that $\lambda$ is bounded on $[0, \infty)$. By the preceding step,

$$
T_{+}(u)=\infty .
$$

Let us fix $\phi \in C_{0}^{\infty}\left(\mathbb{R}^{3}\right)$ such that $\phi \equiv 1$ for $|x| \leq 1$, and $\phi \equiv 0$ for $|x| \geq 2$. For $R>1$, write $\phi_{R}=\phi(x / R), \psi_{R}=x \phi(x / R)$ and

$$
\rho(R)=\sup _{t \in\left(T_{-}(u), T_{+}(u)\right)} \int_{|x| \geq R}\left[\frac{|u|^{2}}{|x|^{2}}+|\nabla u|^{2}+\left|\partial_{t} u\right|^{2}+|u|^{6}\right] d x .
$$

The compactness of $\bar{K}$ and the boundedness of $\lambda$ imply that $\rho(R)$ is finite, and tends to 0 as $R$ goes to infinity. Let

$$
y_{R}(t)=\int_{\mathbb{R}^{3}} \psi_{R} \cdot \nabla u \partial_{t} u d x+\frac{1}{2} \int_{\mathbb{R}^{3}} \varphi_{R} u \partial_{t} u d x .
$$

Then (see [KM08, Lemma 5.3])

$$
y_{R}^{\prime}(t)=-\int_{\mathbb{R}^{3}}\left(\partial_{t} u\right)^{2} d x+\mathcal{O}(\rho(R)) .
$$


Integrating with respect to time, we find that there exists a constant $C>0$, independent of $R$, such that for all $T>0$,

$$
\int_{0}^{T}\left\|\partial_{t} u(t)\right\|_{L^{2}}^{2} d t \leq\left|y_{R}(T)-y_{R}(0)\right|+C T \rho(R) .
$$

Using that, for any fixed $R>0, y_{R}(t)$ is bounded independently of $t$, we get

$$
\lim _{T \rightarrow \infty} \frac{1}{T} \int_{0}^{T}\left\|\partial_{t} u(t)\right\|_{L^{2}}^{2} d t=0
$$

We next show that there exists a sequence $t_{n} \rightarrow \infty$ such that

$$
\lim _{n \rightarrow \infty} \frac{1}{\lambda\left(t_{n}\right)} \int_{t_{n}}^{t_{n}+\lambda\left(t_{n}\right)}\left\|\partial_{t} u(t)\right\|_{L^{2}}^{2} d t=0 .
$$

Indeed, define

$$
\tau_{0}=0, \quad \tau_{n+1}=\tau_{n}+\lambda\left(\tau_{n}\right) .
$$

We first show that $\tau_{n} \rightarrow \infty$. If not, $\tau_{n}$ has a finite limit $\tau_{\infty}=\sum_{n \geq 0} \lambda\left(\tau_{n}\right)$, which shows by continuity of $\lambda$ that $\lambda\left(\tau_{\infty}\right)=0$, a contradiction with the assumption that $\lambda$ takes strictly positive values.

Assuming, towards a contradiction, that no subsequence $\left\{t_{n}\right\}$ of $\left\{\tau_{n}\right\}$ satisfies (6.5), we see that there exists $\varepsilon>0$ such that

$$
\forall n, \quad \int_{\tau_{n}}^{\tau_{n+1}}\left\|\partial_{t} u(t)\right\|_{L^{2}}^{2} d t \geq \varepsilon \lambda\left(\tau_{n}\right) .
$$

Summing up, and using that $\tau_{n+1}=\sum_{k=1}^{n} \lambda\left(\tau_{k}\right)$, we get

$$
\forall n, \quad \frac{1}{\tau_{n+1}} \int_{0}^{\tau_{n+1}}\left|\partial_{t} u(t)\right|_{L^{2}}^{2} d t \geq \varepsilon,
$$

contradicting 6.4). Hence 6.5.

Extracting subsequences, we get $\left(U_{0}, U_{1}\right) \in \dot{H}^{1} \times L^{2}$ such that

$$
\left(\lambda_{n}^{N / 2-1} u\left(t_{n}, \lambda\left(t_{n}\right) x\right), \lambda_{n}^{N / 2} \partial_{t} u\left(t_{n}, \lambda\left(t_{n}\right) x\right)\right) \underset{n \rightarrow \infty}{\longrightarrow}\left(U_{0}, U_{1}\right) .
$$

Let $U$ be the solution of 1.1 with initial conditions $\left(U_{0}, U_{1}\right)$. Let $\theta_{0} \in\left(0, T_{+}(U)\right)$ with $\theta_{0} \leq 1$. Then by Theorem 2.1

$$
\begin{aligned}
& \frac{1}{\theta_{0} \lambda\left(t_{n}\right)} \int_{t_{n}}^{t_{n}+\lambda\left(t_{n}\right)}\left\|\partial_{t} u(t)\right\|_{L^{2}}^{2} d t \geq \frac{1}{\theta_{0} \lambda\left(t_{n}\right)} \int_{t_{n}}^{t_{n}+\theta_{0} \lambda\left(t_{n}\right)}\left\|\partial_{t} u(t)\right\|_{L^{2}}^{2} d t \\
& \quad=\frac{1}{\theta_{0} \lambda\left(t_{n}\right)} \int_{0}^{\theta_{0} \lambda\left(t_{n}\right)}\left\|\partial_{t} U\left(t / \lambda\left(t_{n}\right)\right)\right\|_{L^{2}}^{2} d t+o_{n}(1)=\int_{0}^{\theta_{0}}\left\|\partial_{t} U(s)\right\|_{L^{2}}^{2} d s+o_{n}(1) .
\end{aligned}
$$

By 6.5 , we see that $\partial_{t} U=0$ on $\left[0, \theta_{0}\right]$. By Claim 2.2 $U=W$, which shows that $E\left(U_{0}, U_{1}\right)=E\left(u_{0}, u_{1}\right)=E(W, 0)$. This concludes Step 2. 
Step 3. We next show that $E\left(u_{0}, u_{1}\right)=E(W, 0)$ also if $\lambda$ is unbounded on both intervals $[0, \infty)$ and $(-\infty, 0]$. We will use an argument of [KM06] to reduce to the previous case. We sketch the argument for the sake of completeness. Consider the sequence $\left\{t_{n}\right\}_{n}$,

$$
t_{n}=\inf \left\{t \in[0, \infty): \lambda\left(t_{n}\right)=n\right\} .
$$

By continuity of $\lambda$ and the fact that $\lambda(t)$ tends to $\infty$ as $t$ tends to $\infty$, we see that $t_{n}$ is well-defined for large $n$ and

$$
\lim _{n \rightarrow \infty} t_{n}=\infty, \quad \forall t \in\left[0, t_{n}\right], \lambda(t) \leq \lambda\left(t_{n}\right) .
$$

Extracting subsequences, consider $\left(U_{0}, U_{1}\right)$ such that

$$
\lim _{n \rightarrow \infty}\left(\lambda\left(t_{n}\right)^{N / 2-1} u\left(t_{n}, \lambda\left(t_{n}\right) x\right), \lambda\left(t_{n}\right)^{N / 2} \partial_{t} u\left(t_{n}, \lambda\left(t_{n}\right) x\right)\right)=\left(U_{0}, U_{1}\right) .
$$

Note that we cannot have $\left(U_{0}, U_{1}\right)=(0,0)$ (this would imply, by Claim 2.3 , that $u=$ $0)$. Let $U$ be the solution of 11.1 with initial conditions $\left(U_{0}, U_{1}\right)$. By the arguments of [KM06, proof of Theorem 7.1], we can show, as a consequence of the compactness of $\bar{K}$ and $(6.6)$, that there exists a continuous function $\tilde{\lambda}$ on $\left(T_{-}(U), T_{+}(U)\right)$, bounded on $\left(T_{-}(U), 0\right]$ and such that

$$
\widetilde{K}=\left\{\left(\tilde{\lambda}(t)^{N / 2-1} U(t, \tilde{\lambda}(t) x), \tilde{\lambda}(t)^{N / 2} \partial_{t} U(t, \tilde{\lambda}(t) x)\right): t \in\left(T_{-}(U), T_{+}(U)\right)\right\}
$$

has compact closure in $\dot{H}^{1} \times L^{2}$. By Step 1, $U$ is globally defined. By Step 2, as $\tilde{\lambda}$ is bounded on $(-\infty, 0]$, we find that $E\left(U_{0}, U_{1}\right)=E(W, 0)$. Thus by conservation of the energy of $u, E\left(u_{0}, u_{1}\right)=E(W, 0)$, which concludes this step.

Step 4: Convergence in mean to $W$. By [DM08, Theorem 2], $\|\nabla u(t)\|_{L^{2}}^{2} \geq\|\nabla W\|_{L^{2}}^{2}$ for all $t$ : if not, $u$ would scatter at least in one time direction, contradicting the compactness of $K$.

To show that $u=W$, we will use the arguments of [DM08, Section 3] $]^{2}$ In that section, it is shown in particular that a globally defined solution $u$ of (1.1) of energy $E(W, 0)$, satisfying $\left\|\nabla u_{0}\right\|_{L^{2}}^{2} \leq\|\nabla W\|_{L^{2}}^{2}$ and such that there exists $\lambda(t)$ with $K$ compact, must be equal to $W$ up to the symmetries of $[1.1$. We will quickly check here that the same proof works with a slight modification in the case $\|\nabla u(t)\|_{L^{2}}^{2} \geq\|\nabla W\|_{L^{2}}^{2}$. As usual, we may assume that $\lambda(t)$ is a continuous function of $t$. Let

$$
\mathrm{d}(t)=8 \int\left(\partial_{t} u\right)^{2}+4\left(\int|\nabla u|^{2}-\int|\nabla W|^{2}\right) \geq 0 .
$$

By the characterization of $W$ ([Aub76], [Tal76]), for any $t_{0}, \mathrm{~d}\left(t_{0}\right)=0$ if and only if $u\left(t_{0}\right) \equiv W$ up to the symmetries of the equation. In this case, by uniqueness of the Cauchy problem, $u(t)$ is a stationary solution identically equal to $W$ up to the symmetries.

In this step we show that

$$
\lim _{T \rightarrow \infty} \frac{1}{T} \int_{-T}^{T} \mathrm{~d}(t) d t=0
$$

\footnotetext{
2 In the cited paper, the notation $\lambda(t)$ stands for the function $1 / \lambda(t)$ of the present paper.
} 
Choose a function $\varphi \in C_{0}^{\infty}$ such that $\varphi=1$ if $|x| \leq 1$, and write $\varphi_{R}(x)=\varphi(x / R)$. Let $g_{R}(t)=2 \int u \partial_{t} u \varphi_{R}$ and note that $\left|g_{R}(t)\right| \leq C_{0} R$ for a constant $C_{0}>0$ depending only on $\sup _{t}\left[\left\|\partial_{t} u(t)\right\|_{L^{2}}+\|\nabla u(t)\|_{L^{2}}\right]$. Using that $u$ is solution of (1.1), we get

$$
g_{R}^{\prime}(t)=\mathrm{d}(t)+A_{R}(t)
$$

where

$$
\left|A_{R}(t)\right| \leq \int_{|x| \geq R}\left[\frac{1}{|x|^{2}} u^{2}+u^{6}+|\nabla u|^{2}+\left(\partial_{t} u\right)^{2}\right] .
$$

As in the case $\|\nabla u(t)\|_{L^{2}}<\|\nabla W\|_{L^{2}}$ we will use that $g_{R}$ and $g_{R}^{\prime}$ vanish for $u=W$, and that $\left|g_{R}^{\prime}\right|$ is larger than $\mathrm{d}(t)$ up to the remainder term $A_{R}$. In our case, the definition of $g_{R}$ is slightly different but it will not affect the proof.

Fix a small $\varepsilon>0$. As in the proof of Lemma 3.3 of [DM08], using that $\lambda(t) / t \rightarrow 0$ as $t \rightarrow \pm \infty$, we deduce that there exists a constant $C_{1}$, independent of $\varepsilon$, and a time $t_{1}=t_{1}(\varepsilon)$ such that

$$
\forall T>2 t_{1}(\varepsilon), \forall t \in\left[t_{1}(\varepsilon), T\right], \quad g_{\varepsilon T}^{\prime}(t) \geq \mathrm{d}(t)-C_{1} \varepsilon
$$

integrating between $t_{1}$ and $T$ we find that $T^{-1} \int_{0}^{T} \mathrm{~d}(t) d t$ tends to 0 . The same proof works for negative time, yielding 6.7.

Step 5. In view of 6.8, and refining the bound on $g_{R}(t)$ and the estimate 6.9) on $A_{R}(t)$ by modulating the solution around $W$ for small $\mathrm{d}(t)$, we conclude that there is a constant $C>0$ (depending only on the set $K$ ) such that

$$
\forall \sigma, \tau \in \mathbb{R}, \quad \sigma<\tau \Rightarrow \int_{\sigma}^{\tau} \mathrm{d}(t) d t \leq C\left(\sup _{\sigma \leq t \leq \tau} \lambda(t)\right)(\mathrm{d}(\sigma)+\mathrm{d}(\tau))
$$

(see the proof of Lemma 3.8 in [DM08]). Using compactness and modulation arguments, we get the following control on $\lambda(t)$ (see Lemma 3.10 in [DM08] and its proof):

$$
\sigma+\lambda(\sigma) \leq \tau \Rightarrow|\lambda(\sigma)-\lambda(\tau)| \leq \int_{\sigma}^{\tau} \mathrm{d}(t) d t
$$

Pick two sequences $\sigma_{n} \rightarrow-\infty$ and $\tau_{n} \rightarrow \infty$ such that $\mathrm{d}\left(\sigma_{n}\right) \rightarrow 0$ and $\mathrm{d}\left(\tau_{n}\right) \rightarrow 0$ as $n \rightarrow \infty$. The existence of $\left\{\sigma_{n}\right\}_{n}$ and $\left\{\tau_{n}\right\}_{n}$ is given by 6.7 in Step 4. Let $n_{0}$ be such that $\mathrm{d}\left(\tau_{n_{0}}\right) \leq 1 / 2$. Let us prove that $\lambda$ is bounded. For large $n$, let $t_{n} \in\left[\tau_{n_{0}}, \tau_{n}\right]$ be such that

$$
\lambda\left(t_{n}\right)=\max _{\tau_{n_{0}} \leq t \leq \tau_{n}} \lambda(t)
$$

If $\lambda\left(t_{n}\right) \rightarrow \infty$, then by continuity of $\lambda, t_{n} \rightarrow \infty$. In particular for large $n, \tau_{n_{0}}+\lambda\left(\tau_{n_{0}}\right)$ $\leq t_{n}$, and we can deduce from 6.10 and 6.11 that

$$
\lambda\left(t_{n}\right) \leq \lambda\left(\tau_{n_{0}}\right)+\lambda\left(t_{n}\right)\left(1 / 2+\mathrm{d}\left(\tau_{n}\right)\right),
$$

a contradiction if $\lambda\left(t_{n}\right) \rightarrow \infty$. Thus $\lambda$ is bounded on $[0, \infty)$ and a similar proof yields the boundedness of $\lambda$ on $(-\infty, 0]$. As a consequence of [6.10), we get

$$
\int_{\sigma_{n}}^{\tau_{n}} \mathrm{~d}(t) d t \leq C\left(\mathrm{~d}\left(\sigma_{n}\right)+\mathrm{d}\left(\tau_{n}\right)\right),
$$

which implies that $\mathrm{d}(t)=0$ for all $t$, concluding the sketch of the proof. 


\section{Bounded globally defined solutions are not self-similar}

This section is dedicated to the proof of the following proposition, which will be needed in Section 8 and uses some of the material of Section 3 .

Proposition 7.1. Assume that $N=3$. There exists a constant $\eta_{1}>0$ with the following property. Let $u$ be a spherically symmetric solution of (1.1) such that $T_{+}(u)=\infty$, which does not scatter for positive time and such that

$$
\sup _{t \geq 0}\left[\|\nabla u(t)\|_{L^{2}}^{2}+\left\|\partial_{t} u(t)\right\|_{L^{2}}^{2}\right] \leq\|\nabla W\|_{L^{2}}^{2}+\eta_{1} .
$$

Define

$$
v(t)=\inf \left\{\mu: \int_{|x| \geq \mu}\left[\left|\partial_{t} u(t)\right|^{2}+|\nabla u(t)|^{2}\right] \leq \frac{1}{2} \int|\nabla W|^{2}\right\} .
$$

Then there exists a sequence $t_{n} \rightarrow \infty$ such that

$$
\lim _{n \rightarrow \infty} v\left(t_{n}\right) / t_{n}=0 .
$$

Proof. Assume that (7.3) does not hold. Taking into account the finite speed of propagation, we deduce that there exist $c_{0}, C_{0}$ such that

$$
\forall t \geq 1, \quad c_{0} t \leq v(t) \leq C_{0} t .
$$

Step 1. Let $\mathcal{A}$ be the set of $\left(\begin{array}{c}U_{0} \\ U_{1}\end{array}\right)$ such that there exists $t_{n} \rightarrow \infty$ with

$$
\left(\begin{array}{c}
t_{n}^{1 / 2} u\left(t_{n}, t_{n} x\right) \\
t_{n}^{3 / 2} \partial_{t} u\left(t_{n}, t_{n} x\right)
\end{array}\right) \underset{n \rightarrow \infty}{\longrightarrow}\left(\begin{array}{c}
U_{0} \\
U_{1}
\end{array}\right) \quad \text { weakly in } \dot{H}^{1} \times L^{2} .
$$

In this step we show that there is an $\left(A_{0}, A_{1}\right) \in \mathcal{A}$ with minimal energy, that is, such that

$$
\forall\left(U_{0}, U_{1}\right) \in \mathcal{A}, \quad E\left(A_{0}, A_{1}\right) \leq E\left(U_{0}, U_{1}\right) .
$$

We first show that $\mathcal{A}$ is sequentially closed in $\dot{H}^{1} \times L^{2}$ for the weak topology. Indeed, let $\left(U_{0, n}, U_{1, n}\right) \rightarrow\left(U_{0}, U_{1}\right)$ with $\left(U_{0, n}, U_{1, n}\right) \in \mathcal{A}$. Select a countable family of smooth compactly supported functions $\left\{\left(\varphi_{j}, \psi_{j}\right)\right\}_{j \in \mathbb{N}}$ which is dense in $\dot{H}^{-1} \times L^{2}$. Then for all $k$, there exists $n_{k}$ such that

$$
\left|\int\left(U_{0, n_{k}}-U_{0}\right) \varphi_{j}\right|+\left|\int\left(U_{1, n_{k}}-U_{1}\right) \psi_{j}\right| \leq \frac{1}{k}, \quad j=0, \ldots, k .
$$

Thus there exists $t_{k} \geq k$ such that

$$
\left|\int\left(t_{k}^{1 / 2} u\left(t_{k}, t_{k} x\right)-U_{0}\right) \varphi_{j}\right|+\left|\int\left(t_{k}^{3 / 2} \partial_{t} u\left(t_{k}, t_{k} x\right)-U_{1}\right) \psi_{j}\right| \leq \frac{2}{k}, \quad j=0, \ldots, k .
$$

This shows that $\left(t_{k}^{1 / 2} u\left(t_{k}, t_{k} x\right), t_{k}^{3 / 2} \partial_{t} u\left(t_{k}, t_{k} x\right)\right)$ converges weakly to $\left(U_{0}, U_{1}\right)$ and thus $\left(U_{0}, U_{1}\right) \in \mathcal{A}$. 
We next construct the minimizing element $\left(A_{0}, A_{1}\right)$ of $\mathcal{A}$. Let $\left\{\left(U_{0, n}, U_{1, n}\right)\right\}_{n}$ be a sequence in $\mathcal{A}$ minimizing the energy. As $\left\{\left(U_{0, n}, U_{1, n}\right)\right\}_{n}$ is bounded in $\dot{H}^{1} \times L^{2}$, we can extract from it a subsequence such that

$$
\left(U_{0, n}, U_{1, n}\right) \underset{n \rightarrow \infty}{\longrightarrow}\left(A_{0}, A_{1}\right) \in \mathcal{A} .
$$

Define $\tilde{w}_{0, n}=U_{0, n}-A_{0}, \tilde{w}_{1, n}=U_{1, n}-A_{1}$. Writing after extraction of a subsequence the profile decomposition of the sequence $\left\{\left(U_{0, n}, U_{1, n}\right)\right\}$ and using the Pythagorean expansions 2.14-2.16, we get

$$
\begin{gathered}
\left\|\nabla U_{0, n}\right\|_{L^{2}}^{2}+\left\|U_{1, n}\right\|_{L^{2}}^{2}=\left\|\nabla A_{0}\right\|_{L^{2}}^{2}+\left\|A_{1}\right\|_{L^{2}}^{2}+\left\|\nabla \tilde{w}_{0, n}\right\|_{L^{2}}^{2}+\left\|\tilde{w}_{1, n}\right\|_{L^{2}}^{2}+o_{n}(1), \\
E\left(U_{0, n}, U_{1, n}\right)=E\left(A_{0}, A_{1}\right)+E\left(\tilde{w}_{0, n}, \tilde{w}_{1, n}\right)+o_{n}(1) .
\end{gathered}
$$

By (7.6) and assumption (7.1), we obtain, for large $n$,

$$
\left\|\nabla A_{0}\right\|_{L^{2}}^{2}+\left\|A_{1}\right\|_{L^{2}}^{2}+\left\|\nabla \tilde{w}_{0, n}\right\|_{L^{2}}^{2}+\left\|\nabla \tilde{w}_{1, n}\right\|_{L^{2}}^{2} \leq\|\nabla W\|_{L^{2}}^{2}+2 \eta_{1},
$$

which shows by Claim 2.3 that in (7.7), all the energies are positive. Thus

$$
\inf _{\left(V_{0}, V_{1}\right) \in \mathcal{A}} E\left(V_{0}, V_{1}\right)=\lim _{n \rightarrow \infty} E\left(U_{0, n}, U_{1, n}\right) \geq E\left(A_{0}, A_{1}\right),
$$

implying that $\left(A_{0}, A_{1}\right)$ satisfies 7.5 .

Step 2: Profile decomposition. Choose an arbitrary positive sequence $\left\{\tau_{n}\right\}_{n}$ that tends to $\infty$ and such that

$$
\left(\tau_{n}^{1 / 2} u\left(\tau_{n}, \tau_{n} x\right), \tau_{n}^{3 / 2} \partial_{t} u\left(\tau_{n}, \tau_{n} x\right)\right) \underset{n \rightarrow \infty}{\longrightarrow}\left(A_{0}, A_{1}\right) \quad \text { weakly in } \dot{H}^{1} \times L^{2},
$$

where $\left(A_{0}, A_{1}\right)$ is the minimal element of $\mathcal{A}$ defined in Step 1.

Extracting a subsequence from $\left\{\tau_{n}\right\}_{n}$, we can assume that their exists a profile decomposition $\left\{U_{\mathrm{L}}^{j}\right\},\left\{\lambda_{j, n}, t_{j, n}\right\}$ associated to the sequence $\left\{\left(u\left(\tau_{n}\right), \partial_{t} u\left(\tau_{n}\right)\right)\right\}_{n}$.

Reordering the profiles, we may assume

$$
\left\|\nabla U_{0}^{1}\right\|_{L^{2}}^{2}+\left\|U_{1}^{1}\right\|_{L^{2}}^{2}=\sup _{j \geq 1}\left[\left\|\nabla U_{0}^{j}\right\|_{L^{2}}^{2}+\left\|U_{1}^{j}\right\|_{L^{2}}^{2}\right] .
$$

We remark that

$$
\left\|\nabla U_{0}^{1}\right\|_{L^{2}}^{2}+\left\|U_{1}^{1}\right\|_{L^{2}}^{2} \geq \frac{2}{3}\|\nabla W\|_{L^{2}}^{2} .
$$

If not, the result of [KM08] would imply that all nonlinear profiles $U^{j}$ scatter, showing by Proposition 2.8 that $u$ scatters for both positive and negative times, which contradicts our assumption.

As a consequence, we deduce from (7.1) and again the result of [KM08] that for all $j \geq 2$, the nonlinear profile $U^{j}$ scatters both for positive and for negative time.

Extracting subsequences and time-translating $U_{\mathrm{L}}^{1}$ if necessary, we may distinguish three cases: 
(a) $\lim _{n \rightarrow \infty} \frac{-t_{1, n}}{\lambda_{1, n}}=\infty$

(b) $\lim _{n \rightarrow \infty} \frac{-t_{1, n}}{\lambda_{1, n}}=-\infty$.

(c) $\forall n, t_{1, n}=0$.

Case (a) is clearly excluded, as it would imply by Proposition 2.8 that $u$ scatters for positive time, contradicting our assumptions.

Assume that $(\mathrm{b})$ holds. Then the nonlinear solution $U^{1}$ scatters for negative time. Precisely, by definition of $U^{1}$,

$$
\lim _{t \rightarrow-\infty}\left[\left\|U_{\mathrm{L}}^{1}(t)-U^{1}(t)\right\|_{\dot{H}^{1}}+\left\|\partial_{t} U_{\mathrm{L}}^{1}(t)-\partial_{t} U^{1}(t)\right\|_{L^{2}}\right]=0 .
$$

Furthermore, by Proposition 2.8 , denoting as usual by $U_{n}^{j}$ the rescaled profiles (see Notation 2.6,

$$
\begin{aligned}
u(0)= & \frac{1}{\lambda_{1, n}^{1 / 2}} U_{\mathrm{L}}^{1}\left(\frac{-t_{1, n}-\tau_{n}}{\lambda_{1, n}}, \frac{x}{\lambda_{1, n}}\right)+\sum_{j=2}^{J} U_{n}^{j}\left(-\tau_{n}, x\right)+w_{n}^{J}\left(-\tau_{n}\right)+r_{n}^{J}\left(-\tau_{n}\right), \\
\partial_{t} u(0)= & \frac{1}{\lambda_{1, n}^{3 / 2}} \partial_{t} U_{\mathrm{L}}^{1}\left(\frac{-t_{1, n}-\tau_{n}}{\lambda_{1, n}}, \frac{x}{\lambda_{1, n}}\right) \\
& +\sum_{j=2}^{J} \partial_{t} U_{n}^{j}\left(-\tau_{n}, x\right)+\partial_{t} w_{n}^{J}\left(-\tau_{n}\right)+\partial_{t} r_{n}^{J}\left(-\tau_{n}\right) .
\end{aligned}
$$

Let $v_{n}(t)=S_{\mathrm{L}}(t)\left(\lambda_{1, n}^{1 / 2} u\left(0, \lambda_{1, n} x\right), \lambda_{1, n}^{3 / 2} u\left(0, \lambda_{1, n} x\right)\right)$. By orthogonality of the parameters $\left\{\lambda_{j, n}, t_{j, n}\right\}$, the developments 7.11, 7.12 imply

$$
\left(v_{n}\left(\frac{t_{1, n}+\tau_{n}}{\lambda_{1, n}}\right), \partial_{t} v_{n}\left(\frac{t_{1, n}+\tau_{n}}{\lambda_{1, n}}\right)\right) \underset{n \rightarrow \infty}{\longrightarrow}\left(U_{0}^{1}, U_{1}^{1}\right) \quad \text { in } \dot{H}^{1} \times L^{2}
$$

since $\left(t_{1, n}+\tau_{n}\right) / \lambda_{1, n} \rightarrow \infty$ this would imply $\left(U_{0}^{1}, U_{1}^{1}\right)=(0,0)$, a contradiction.

Step 3: Compact main profile. It remains to consider case (c). By (7.4),

$$
\begin{aligned}
& \int_{C_{0} \tau_{n} \leq|x|}\left[\left|\nabla u\left(\tau_{n}, x\right)\right|^{2}+\left|\partial_{t} u\left(\tau_{n}, x\right)\right|^{2}\right] d x \\
& \quad \leq \frac{1}{2} \int|\nabla W|^{2} \leq \int_{c_{0} \tau_{n} \leq|x|}\left[\left|\nabla u\left(\tau_{n}, x\right)\right|^{2}+\left|\partial_{t} u\left(\tau_{n}, x\right)\right|^{2}\right] d x .
\end{aligned}
$$

This shows by assumption 7.1 ,

$$
\int_{|x| \leq c_{0} \tau_{n}}\left[\left|\nabla u\left(\tau_{n}, x\right)\right|^{2}+\left|\partial_{t} u\left(\tau_{n}, x\right)\right|^{2}\right] d x \leq \frac{1}{2} \int|\nabla W|^{2}+\eta_{1},
$$


and thus by 7.10 (using that $t_{1, n}=0$ ), $\lambda_{1, n} \approx \tau_{n}$. Extracting subsequences and rescaling $U^{1}$ we may assume that $\lambda_{1, n}=\tau_{n}$. Then by $(7.8)$,

$$
U_{0}^{1}=A_{0}, \quad U_{1}^{1}=A_{1} .
$$

We will show that $T_{-}\left(U^{1}\right)=-1$ and that

$$
K=\left\{\left(\begin{array}{c}
(1+t)^{1 / 2} U^{1}(t,(1+t) x) \\
(1+t)^{3 / 2} \partial_{t} U^{1}(t,(1+t) x)
\end{array}\right): t \in(-1,0]\right\}
$$

has compact closure in $\dot{H}^{1} \times L^{2}$. This type of self-similar solution is excluded by [KM08, Section 6]. Let $\sigma \in\left(T_{-}\left(U_{1}\right), 0\right)$. Then by Proposition 2.8

$$
\begin{gathered}
u\left(\tau_{n}+\sigma \tau_{n}\right)=\frac{1}{\tau_{n}^{1 / 2}} U^{1}\left(\sigma, \frac{x}{\tau_{n}}\right)+\sum_{j=2}^{J} U_{n}^{j}\left(\sigma \tau_{n}\right)+w_{n}^{J}\left(\sigma \tau_{n}\right)+r_{n}^{J}\left(\sigma \tau_{n}\right) \\
\partial_{t} u\left(\tau_{n}+\sigma \tau_{n}\right)=\frac{1}{\tau_{n}^{3 / 2}} \partial_{t} U^{1}\left(\sigma, \frac{x}{\tau_{n}}\right)+\sum_{j=2}^{J} \partial_{t} U_{n}^{j}\left(\sigma \tau_{n}\right)+\partial_{t} w_{n}^{J}\left(\sigma \tau_{n}\right)+\partial_{t} r_{n}^{J}\left(\sigma \tau_{n}\right) .
\end{gathered}
$$

Let

$$
Z_{n}^{J}(t)=\sum_{j=2}^{J} U_{n}^{j}(t)+w_{n}^{J}(t)
$$

By Remark 2.10, for large $J$ and $n$ we have

$$
\left\|\nabla_{t, x} U^{1}(\sigma)\right\|_{L^{2}}^{2}+\left\|\nabla_{t, x} Z_{n}^{J}\left(\sigma \tau_{n}\right)\right\|_{L^{2}}^{2} \leq\left\|\nabla_{t, x} u\left(\tau_{n}+\sigma \tau_{n}\right)\right\|_{L^{2}}^{2}+\eta_{1} .
$$

By assumption (7.1) and using (7.10), we get

$$
\left\|\nabla_{t, x} Z_{n}^{J}\left(\sigma \tau_{n}\right)\right\|_{L^{2}}^{2} \leq \frac{1}{3}\|\nabla W\|_{L^{2}}^{2}+2 \eta_{1} .
$$

By (7.4) and the triangle inequality, we deduce that for large $J$ and $n$,

$$
\begin{aligned}
& \sqrt{\frac{1}{2} \int|\nabla W|^{2}} \leq \sqrt{\int_{c_{0}(1+\sigma) \tau_{n} \leq|x|}\left|\nabla_{t, x} u\left((1+\sigma) \tau_{n}, x\right)\right|^{2} d x} \\
& \leq \sqrt{\int_{c_{0}(1+\sigma) \tau_{n} \leq|x|} \frac{1}{\tau_{n}^{3}}\left|\nabla_{t, x} U^{1}\left(\sigma, \frac{x}{\tau_{n}}\right)\right|^{2} d x}+\sqrt{\int_{c_{0}(1+\sigma) \tau_{n} \leq|x|}\left|\nabla_{t, x} Z_{n}^{J}\left(\sigma \tau_{n}, x\right)\right|^{2} d x}+\eta_{1} .
\end{aligned}
$$

Thus by (7.18), and if $\eta_{1}$ is chosen small enough so that the left hand side inequality holds,

$$
\left(2 \eta_{1}\right)^{1 / 2} \leq \sqrt{\frac{1}{2} \int|\nabla W|^{2}}-\sqrt{\frac{1}{3} \int|\nabla W|^{2}+2 \eta_{1}}-\eta_{1} \leq \sqrt{\int_{c_{0}(1+\sigma) \leq|x|}\left|\nabla_{t, x} U^{1}(\sigma)\right|^{2}} .
$$


Using again assumption 7.1 , we obtain

$$
\forall \sigma \in(-1,0), \quad \int_{|x| \leq c_{0}(1+\sigma)}\left|\nabla_{t, x} U^{1}(\sigma)\right|^{2} \leq \int_{\mathbb{R}^{3}}|\nabla W|^{2}-\eta_{1} .
$$

In view of 3.4 in Theorem 3.2. we must have $T_{-}\left(U^{1}\right) \leq-1$. We cannot have $T_{-}\left(U^{1}\right)$ $<-1$ because (7.16, (7.17) with $\sigma=-1$ would give a nontrivial profile decomposition for $\left(u(0), \partial_{t} u(0)\right)$, a contradiction. Thus $T_{-}\left(U^{1}\right)=-1$.

Next, note that by the developments (7.16, 7.17), we have

$$
\left(\tau_{n}^{1 / 2} u\left((1+\sigma) \tau_{n}, \tau_{n} \cdot\right), \tau_{n}^{3 / 2} u\left((1+\sigma) \tau_{n}, \tau_{n} \cdot\right)\right) \underset{n \rightarrow \infty}{\longrightarrow}\left(U^{1}(\sigma), \partial_{t} U^{1}(\sigma)\right) .
$$

This shows that

$\forall \sigma \in\left(-1, T_{+}\left(U^{1}\right)\right), \quad\left((1+\sigma)^{1 / 2} U^{1}(\sigma,(1+\sigma) \cdot),(1+\sigma)^{3 / 2} \partial_{t} U^{1}(\sigma,(1+\sigma) \cdot)\right) \in \mathcal{A}$.

We next show that $K$ defined by (7.15) has compact closure in $\dot{H}^{1} \times L^{2}$. Indeed, let $t_{n}$ be a sequence that goes to -1 and assume after extraction that (weakly in $\dot{H}^{1} \times L^{2}$ )

$$
\left(\left(1+t_{n}\right)^{1 / 2} U^{1}\left(t_{n},\left(1+t_{n}\right) \cdot\right),\left(1+t_{n}\right)^{3 / 2} \partial_{t} U^{1}\left(t_{n},\left(1+t_{n}\right) \cdot\right)\right) \stackrel{n \rightarrow \infty}{\longrightarrow}\left(\widetilde{U}_{0}, \widetilde{U}_{1}\right) .
$$

Then by $(7.20)$ and the fact that $\mathcal{A}$ is closed for the weak topology, $\left(\widetilde{U}_{0}, \widetilde{U}_{1}\right) \in \mathcal{A}$. In particular, using that $\left(U_{0}^{1}, U_{1}^{1}\right)=\left(A_{0}, A_{1}\right)$ has minimal energy in $\mathcal{A}$,

$$
0<E\left(U_{0}^{1}, U_{1}^{1}\right) \leq E\left(\widetilde{U}_{0}, \widetilde{U}_{1}\right) .
$$

We must show that 7.21 is (at least for a subsequence) a strong convergence. For this, consider, after extraction, a profile decomposition for the sequence

$$
\left(U^{1}\left(t_{n}, x\right)-\frac{1}{\left(1+t_{n}\right)^{1 / 2}} \widetilde{U}_{0}\left(\frac{x}{1+t_{n}}\right), \partial_{t} U^{1}\left(t_{n}, x\right)-\frac{1}{\left(1+t_{n}\right)^{3 / 2}} \widetilde{U}_{1}\left(\frac{x}{1+t_{n}}\right)\right) .
$$

Denote the profiles by $V_{\mathrm{L}}^{j}$, the parameters by $s_{j, n}$, and $v_{j, n}$ and the remainders by $\tilde{w}_{n}^{J}$. By the Pythagorean expansion of the energy

$E\left(U_{0}^{1}, U_{1}^{1}\right)=E\left(\tilde{U}_{0}, \widetilde{U}_{1}\right)+\sum_{j=1}^{J} E\left(V_{\mathrm{L}}^{j}\left(\frac{-s_{j, n}}{v_{j, n}}\right), \partial_{t} V_{\mathrm{L}}^{j}\left(\frac{-s_{j, n}}{v_{j, n}}\right)\right)+E\left(\tilde{w}_{0, n}^{J}, \tilde{w}_{1, n}^{J}\right)+o_{n}(1)$.

By Claim 2.3, all the energies are positive in this expansion. By 7.22$), E\left(\widetilde{U}_{0}, \widetilde{U}_{1}\right)=$ $E\left(U_{0}^{1}, U_{1}^{1}\right)$, and thus using Claim 2.3 again, $V_{\mathrm{L}}^{j}=0$ for all $j \geq 1$ and $\left\|\nabla \tilde{w}_{0, n}^{J}\right\|_{L^{2}}+$ $\left\|\tilde{w}_{1, n}^{J}\right\|_{L^{2}}$ tends to 0 as $n$ tends to infinity, concluding the proof of the compactness of $\bar{K}$ in $\dot{H}^{1} \times L^{2}$ and yielding the desired contradiction. Note that in this last argument, we only needed the profile decomposition, for a fixed $J$, to show that the weak convergence (7.21) and the inequality (7.22) imply the strong convergence. The proof of Proposition 7.1 is complete. 


\section{Proof of the main result}

In this section we prove Theorem 1 .

Assume that $N=3$ and that $u$ is a spherically symmetric type II blow-up solution such that

$$
\sup _{\tau_{0} \leq t<1}\left[\|\nabla u(t)\|_{L^{2}}^{2}+\left\|\partial_{t} u(t)\right\|_{L^{2}}^{2}\right] \leq\|\nabla W\|_{L^{2}}^{2}+\eta_{0}
$$

The proof of Theorem 1 takes several steps. Consider the singular part $a$ of $u$ given by Definition 3.3. In $\$ 8.1$, we show that a profile decomposition of a sequence $\left\{\left(a\left(\tau_{n}\right), \partial_{t} a\left(\tau_{n}\right)\right)\right\}$ with $\tau_{n} \rightarrow 1^{-}$admits a large profile which is compact up to scaling. In $\$ 8.2$. we show that, at least for a time sequence, the concentration is not self-similar, i.e. $u$ satisfies the assumptions of Section 5 In $\$ 8.3$, we show that $a(t)$ is compact in the energy space up to a scaling parameter. In $\$ 8.4$ it is proven that the only limit, as $t$ tends to 1 , of $a(t)$ up to scaling is $W$. We then conclude the proof of the theorem.

\subsection{Compactness of the main profile}

Lemma 8.1. Assume that $N=3$ and that 8.1 holds. Choose a sequence $\tau_{n} \rightarrow 1^{-}, a$ profile decomposition $\left\{U^{j}\right\},\left\{\lambda_{j, n}, t_{j, n}\right\}$ associated to $\left\{\left(a\left(\tau_{n}\right), \partial_{t} a\left(\tau_{n}\right)\right)\right\}$ and reorder the profiles (after extraction) so that 3.11 holds. Then all the profiles $U^{j}, j \geq 2$, scatter. Furthermore $U^{1}$ does not scatter for positive or negative time,

$$
\left\|U_{0}^{1}\right\|_{\dot{H}^{1}}^{2}+\left\|U_{1}^{1}\right\|_{\dot{H}^{1}}^{2} \geq \frac{2}{3}\|\nabla W\|_{L^{2}}^{2},
$$

and the sequence $\left\{-t_{1, n} / \lambda_{1, n}\right\}_{n}$ is bounded.

In other words, the largest profile is compact up to modulation and we may assume that $t_{1, n}=0$ for all $n$.

Proof. The inequality (8.2) follows from Lemma 3.6. The assumption (8.1) implies that for all $j \geq 2,\left\|U_{1}^{j}\right\|_{L^{2}}^{2}+\left\|\nabla U_{0}^{j}\right\|_{L^{2}}^{2} \leq \frac{1}{3}\|\nabla W\|_{L^{2}}^{2}+\eta_{0}$. Thus all nonlinear profiles $U^{j}$, $j \geq 2$, scatter both forward and backward in time. To conclude the proof, it is sufficient to show that $U^{1}$ does not scatter forward or backward in time, which would imply that $\left\{-t_{1, n} / \lambda_{1, n}\right\}_{n}$ is bounded. Assume that $U^{1}$ is globally defined and scatters forward in time. Then, by Proposition 2.8, $u$ is globally defined and scatters forward in time, a contradiction. It remains to exclude the case when $U^{1}$ is globally defined and scatters backward in time. By Proposition 2.8 again, we find that for $t<0$,

$$
u\left(\tau_{n}+t, x\right)=v\left(\tau_{n}+t, x\right)+\sum_{j=1}^{J} U_{n}^{j}(t, x)+w_{n}^{J}(t, x)+r_{n}^{J}(t, x),
$$

where

$$
\lim _{J \rightarrow \infty} \limsup _{n \rightarrow \infty}\left[\left\|r_{n}^{J}\right\|_{S(-\infty, 0)}+\sup _{t \in(-\infty, 0)}\left(\left\|\nabla r_{n}^{J}(t)\right\|_{L^{2}}+\left\|\partial_{t} r_{n}^{J}(t)\right\|_{L^{2}}\right)\right]=0 .
$$


The solution $U^{1}$ scatters backward, but not forward in time. By [KM08], this implies that $E\left(U_{0}^{1}, U_{1}^{1}\right) \geq E(W, 0)$. As a consequence, for all $t$ in the domain of existence of $U^{1}$,

$$
\left\|\nabla U^{1}(t)\right\|_{L^{2}}^{2}+\left\|\partial_{t} U^{1}(t)\right\|_{L^{2}}^{2} \geq 2 E\left(U_{0}^{1}, U_{1}^{1}\right) \geq 2 E(W, 0)=\frac{2}{3}\|\nabla W\|_{L^{2}}^{2} .
$$

Let $t_{0} \in\left(\tau_{0}, 1\right)$, where $\tau_{0}$ is defined in 8.1 . Taking $t=t_{0}-\tau_{n}<0$ in the preceding decomposition, we obtain, for large $n$,

$$
\begin{array}{r}
u\left(t_{0}, x\right)=\frac{1}{\lambda_{1, n}^{1 / 2}} U^{1}\left(\frac{t_{0}-\tau_{n}-t_{1, n}}{\lambda_{1, n}}, \frac{x-x_{1, n}}{\lambda_{1, n}}\right)+R_{0, n}(x), \\
\partial_{t} u\left(t_{0}, x\right)=\frac{1}{\lambda_{1, n}^{3 / 2}} \partial_{t} U^{1}\left(\frac{t_{0}-\tau_{n}-t_{1, n}}{\lambda_{1, n}}, \frac{x-x_{1, n}}{\lambda_{1, n}}\right)+R_{1, n}(x),
\end{array}
$$

where by Pythagorean expansion, $\left\|\nabla R_{0, n}\right\|_{L^{2}}^{2}+\left\|R_{1, n}\right\|_{L^{2}}^{2} \leq \frac{1}{3}\|\nabla W\|_{L^{2}}^{2}+\eta_{0}$. By 8.3 , we conclude that $\left\{\left(u\left(t_{0}\right), \partial_{t} u\left(t_{0}\right)\right)\right\}_{n}$, considered as a sequence in $n$, admits a nontrivial profile decomposition (recall that $\lambda_{1, n} \rightarrow 0$ ), a contradiction. The proof is complete.

\subsection{Existence of a sequence avoiding self-similar blow-up}

Proposition 8.2. Assume $N=3$ and let $u$ be a radial solution satisfying 8.1). Then there exist $\left\{\tau_{n}\right\}_{n},\left\{\mu_{n}\right\}_{n}$ with

$$
\tau_{n} \rightarrow 1^{-}, \quad 0<\mu_{n} \ll 1-\tau_{n} \quad \text { as } n \rightarrow \infty
$$

such that

$$
\lim _{n \rightarrow \infty} \int_{|x| \geq \mu_{n}}\left[\left(\partial_{t} a\left(\tau_{n}, x\right)\right)^{2}+\left|\nabla a\left(\tau_{n}, x\right)\right|^{2}+\frac{1}{|x|^{2}}\left(a\left(\tau_{n}, x\right)\right)^{2}\right] d x=0 .
$$

Corollary 8.3.

$$
\lim _{t \rightarrow 1^{-}} E\left(a(t), \partial_{t} a(t)\right)=E(W, 0) .
$$

Proof of Corollary 8.3 By Propositions 8.2 and 5.1 (replacing $u$ by $-u$ if necessary), there exists a sequence $\tau_{n} \rightarrow 1^{-}$a sequence $\lambda_{n} \rightarrow 0$ such that

$$
\begin{aligned}
a\left(\tau_{n}, x\right) & =\frac{1}{\lambda_{n}^{1 / 2}} W\left(\frac{x}{\lambda_{n}}\right)+w_{0, n}, \\
\partial_{t} a\left(\tau_{n}, x\right) & =o(1) \quad \text { in } L^{2} \text { as } n \rightarrow \infty,
\end{aligned}
$$

where, denoting by $w_{n}$ the solution of 2.1] with initial condition $\left(w_{0, n}, 0\right)$,

$$
\lim _{n \rightarrow \infty}\left\|w_{n}\right\|_{S(-\infty, \infty)}=0
$$

Step 1. We first show

$$
\lim _{n \rightarrow \infty}\left\|w_{0, n}\right\|_{\dot{H}^{1}}=0 .
$$


Let us mention that this step still works, with a small refinement, after replacing the assumption 8.1 by the more general 4.1 .

Assume that 8.6 does not hold. Extracting a subsequence in $n$, we can assume that there exists $\varepsilon_{0}>0$ and, for all $n, r_{n}>0$ such that

$$
\int_{|x| \geq r_{n}}\left|\nabla w_{0, n}(x)\right|^{2} d x \geq \varepsilon_{0}
$$

Then, arguing as in the proof of Proposition 4.4 (see 4.40), we find that for large $n$,

$$
\int_{r_{n}}^{\infty}\left|\partial_{r}\left(r w_{0, n}\right)(r)\right|^{2} d r \geq \frac{\varepsilon_{0}}{2} .
$$

Next, the fact that $w_{n}(t)=w_{n}(-t)$ and Lemma 4.2 imply that for large $n$, for all $T>0$,

$$
\int_{|x| \geq r_{n}+T \lambda_{n}}\left|\nabla_{t, x} w_{n}\left(-T \lambda_{n}, x\right)\right|^{2} d x \geq \frac{\varepsilon_{0}}{4} .
$$

By Proposition 2.8, we have

$$
\left\{\begin{array}{l}
a\left(\tau_{n}-T \lambda_{n}\right)=\frac{1}{\lambda_{n}^{1 / 2}} W\left(\frac{x}{\lambda_{n}}\right)+w_{n}\left(-T \lambda_{n}\right)+o_{n}(1) \quad \text { in } \dot{H}^{1} \\
\partial_{t} a\left(\tau_{n}-T \lambda_{n}\right)=\partial_{t} w_{n}\left(-T \lambda_{n}\right)+o_{n}(1) \text { in } L^{2}
\end{array}\right.
$$

Combining this with 8.7 we see that there exists an increasing sequence $\left\{n_{k}\right\}$ such that $\tau_{n_{k}}-k \lambda_{n_{k}} \geq 0$ and

$$
\int_{|x| \geq r_{n_{k}}+k \lambda_{n_{k}}}\left|\nabla_{t, x} a\left(\tau_{n_{k}}-k \lambda_{n_{k}}, x\right)\right|^{2} d x \geq \frac{\varepsilon_{0}}{8} .
$$

In view of $\left[8.8\right.$, this contradicts Proposition 4.4 (here $\rho_{1, n}=\lambda_{n}$ ). Step 1 is complete.

Step 2. By Step 1,

$$
\lim _{n \rightarrow \infty} E\left(a\left(\tau_{n}\right), \partial_{t} a\left(\tau_{n}\right)\right)=E(W, 0)
$$

Note that

$$
E\left(u(t), \partial_{t} u(t)\right)=E\left(v(t), \partial_{t} v(t)\right)+E\left(a(t), \partial_{t} a(t)\right)+o(1) \quad \text { as } t \rightarrow 1^{-},
$$

which shows by conservation of energy for $u$ and $v$ that $E\left(a(t), \partial_{t} a(t)\right)$ has a limit as $t \rightarrow 1^{-}$, concluding the proof of Corollary 8.3 .

Proof of Proposition 8.2 By Hardy's inequality

$$
\int_{|x| \geq R} \frac{1}{|x|^{2}}(a(t, x))^{2} d x \leq C \int_{|x| \geq R}|\nabla a(t, x)|^{2} d x,
$$

so that we only need to show that there exist sequences $\left\{\mu_{n}\right\}$ and $\left\{\tau_{n}\right\}$ as in the proposition such that

$$
\lim _{n \rightarrow \infty} \int_{|x| \geq \mu_{n}}\left[\left(\partial_{t} a\left(\tau_{n}, x\right)\right)^{2}+\left|\nabla a\left(\tau_{n}, x\right)\right|^{2}\right] d x=0 .
$$


If this does not hold, there exist $\alpha>0$ and $\varepsilon_{0}>0$ such that

$$
\forall t \in(0,1), \quad \int_{|x| \geq \alpha(1-t)}\left[\left|\partial_{t} a(t, x)\right|^{2}+|\nabla a(t, x)|^{2}\right] d x \geq \varepsilon_{0} .
$$

Step 1. We first show that there exists $\beta>0$ such tha ${ }^{3}$

$$
\liminf _{t \rightarrow 1^{-}} \int_{|x| \geq \beta(1-t)}\left[\left|\partial_{t} a(t, x)\right|^{2}+|\nabla a(t, x)|^{2}\right] d x \geq \frac{2}{3}\|\nabla W\|_{L^{2}}^{2} .
$$

Indeed, assume that 8.10 does not hold, i.e. that there exist sequences $\tau_{n} \rightarrow 1^{-}$and $\beta_{n} \rightarrow 0^{+}$such that

$$
\int_{|x| \geq \beta_{n}\left(1-\tau_{n}\right)}\left[\left|\partial_{t} a\left(\tau_{n}, x\right)\right|^{2}+\left|\nabla a\left(\tau_{n}, x\right)\right|^{2}\right] d x \leq \frac{2}{3}\|\nabla W\|_{L^{2}}^{2}-\varepsilon_{1} .
$$

After extraction, pick a profile decomposition $\left\{U^{j}\right\},\left\{t_{j, n}, \lambda_{j, n}\right\}$ for $\left\{\left(a\left(\tau_{n}\right), \partial_{t} a\left(\tau_{n}\right)\right)\right\}_{n}$. Reordering the profiles, we assume 3.11), i.e. that $U^{1}$ is the largest profile in the energy space. By Lemma 8.1. we may assume that $t_{1, n}=0$, and the norm of $\left(U_{0}^{1}, U_{1}^{1}\right)$ in the energy space is bounded from below (see 8.2 ).

Let $\varepsilon_{2}>0$ to be specified later. By Proposition 3.8 , there exists $T \in\left(0, T_{+}\left(U^{1}\right)\right)$ such that

Then by Proposition 2.8 .

$$
\left\|\nabla U^{1}(T)\right\|_{L^{2}}^{2}+\left\|\partial_{t} U^{1}(T)\right\|_{L^{2}}^{2} \geq\|\nabla W\|_{L^{2}}^{2}-\varepsilon_{2}
$$

$$
\begin{gathered}
a\left(\tau_{n}+\lambda_{1, n} T\right)=\sum_{j=1}^{J} U_{n}^{j}\left(\lambda_{1, n} T\right)+w_{n}^{J}\left(\lambda_{1, n} T\right)+r_{n}^{J}\left(\lambda_{1, n} T\right), \\
\partial_{t} a\left(\tau_{n}+\lambda_{1, n} T\right)=\sum_{j=1}^{J} \partial_{t} U_{n}^{j}\left(\lambda_{1, n} T\right)+\partial_{t} w_{n}^{J}\left(\lambda_{1, n} T\right)+\partial_{t} r_{n}^{J}\left(\lambda_{1, n} T\right) .
\end{gathered}
$$

The rescaled profiles $U_{n}^{j}$ are defined as usual (see Notation 2.6. Note that $\left\|\nabla U^{1}\left(\lambda_{1, n} T\right)\right\|_{L^{2}}^{2}+\left\|\partial_{t} U^{1}\left(\lambda_{1, n} T\right)\right\|_{L^{2}}^{2}=\left\|\nabla U_{n}^{1}(T)\right\|_{L^{2}}^{2}+\left\|\partial_{t} U_{n}^{1}(T)\right\|_{L^{2}}^{2} \geq\|\nabla W\|_{L^{2}}^{2}-\varepsilon_{2}$. Combining this with 8.1 , 8.12, 8.13 and the orthogonality of the parameters, we get

$$
\sum_{j=2}^{J}\left\|\nabla_{t, x} U^{j}\left(\frac{\lambda_{1, n} T-t_{j, n}}{\lambda_{j, n}}\right)\right\|_{L^{2}}^{2}+\left\|\nabla_{t, x} w_{n}^{J}\left(\lambda_{1, n} T\right)\right\|_{L^{2}}^{2} \leq \eta_{0}+\varepsilon_{2} .
$$

And thus using the conservation of energy,

$$
\sum_{j=2}^{J} E\left(U_{0}^{j}, U_{j}^{1}\right) \leq \frac{1}{2}\left(\eta_{0}+\varepsilon_{2}\right)
$$

\footnotetext{
3 We could replace $\frac{2}{3}\|\nabla W\|_{L^{2}}^{2}$ by $\|\nabla W\|_{L^{2}}^{2}-C \eta_{0}$ for some large positive constant $C$, where $\eta_{0}$ is given by 8.1 .
} 
Take $\eta_{0}$ and $\varepsilon_{2}$ so small that $\frac{1}{2}\left(\eta_{0}+\varepsilon_{2}\right) \leq \frac{1}{3} \delta_{1}^{2}$, where $\delta_{1}=\delta_{1}\left(2\|\nabla W\|_{L^{2}}^{2}\right)$ is given by Corollary 4.3 Then $U^{1}$ is the unique large profile, i.e., with the notations of $\$ 4.2, J_{0}=1$. Assume that $\lambda_{1, n}=o_{n}\left(1-\tau_{n}\right)$. Then by Proposition 4.4 we would obtain

$$
\lim _{R \rightarrow \infty} \limsup _{n \rightarrow \infty} \int_{|x| \geq R \lambda_{1, n}}\left[\left|\nabla a\left(\tau_{n}\right)\right|^{2}+\left(\partial_{t} a\left(\tau_{n}\right)\right)^{2}\right] d x=0,
$$

a contradiction with 8.9 . Thus $1-\tau_{n} \approx \lambda_{1, n}$. Choose a sequence $\left\{\tilde{\beta}_{n}\right\}$ such that $\beta_{n} \ll$ $\tilde{\beta}_{n} \ll 1$. Let $\chi \in C^{\infty}\left(\mathbb{R}^{3}\right)$ be such that $\chi(x)=1$ if $|x| \geq 2$ and $\chi(x)=0$ is $|x| \leq 1$. By Remark2.10

$$
\begin{aligned}
\int_{|x| \geq \beta_{n}\left(1-\tau_{n}\right)}\left[\left|\nabla a\left(\tau_{n}, x\right)\right|^{2}+\right. & \left.\left|\partial_{t} a\left(\tau_{n}, x\right)\right|^{2}\right] \\
& \geq \int \chi\left(\frac{x}{\tilde{\beta}_{n}\left(1-\tau_{n}\right)}\right)\left[\left|\nabla a\left(\tau_{n}, x\right)\right|^{2}+\left|\partial_{t} a\left(\tau_{n}, x\right)\right|^{2}\right] \\
& \geq \int \chi\left(\frac{\lambda_{1, n} y}{\tilde{\beta}_{n}\left(1-\tau_{n}\right)}\right)\left[\left|\nabla U_{0}^{1}(y)\right|^{2}+\left|U_{1}^{1}(y)\right|^{2}\right] d y \\
& \underset{n \rightarrow \infty}{\longrightarrow} \int\left[\left|\nabla U_{0}^{1}(y)\right|^{2}+\left|U_{1}^{1}(y)\right|^{2}\right] d y \geq \frac{2}{3}\|\nabla W\|_{L^{2}}^{2} .
\end{aligned}
$$

This contradicts 8.11 and concludes Step 1.

Step 2: End of the argument. Let, for $t \in[0,1)$,

$$
\mu(t)=\inf \left\{\mu: \int_{|x| \leq \mu}\left[\left|\partial_{t} a(t)\right|^{2}+|\nabla a(t)|^{2}\right] \geq \frac{2}{5} \int|\nabla W|^{2}\right\} .
$$

By Step 1 and assumption 8.1 ,

$$
\beta(1-t) \leq \mu(t) \leq 1-t .
$$

Take any sequence $\tau_{n} \rightarrow 1^{-}$such that $\left(a\left(\tau_{n}\right), \partial_{t} a\left(\tau_{n}\right)\right)$ admits a profile decomposition. By Lemma 8.1 and Step 1, we may assume, after extraction,

$$
\left\|\nabla U_{0}^{1}\right\|_{L^{2}}^{2}+\left\|U_{0}^{1}\right\|_{L^{2}}^{2} \geq \frac{2}{3}\|\nabla W\|_{L^{2}}^{2}, \quad t_{1, n}=0, \quad \lambda_{1, n}=\mu\left(\tau_{n}\right) \approx 1-\tau_{n} .
$$

Furthermore, the solution $U^{1}$ does not scatter forward or backward in time. Let $\varepsilon_{3}=$ $\beta^{2} / 2$, where $\beta$ is given by 8.15 . By Proposition 7.1 (if $T_{-}\left(U^{1}\right)=-\infty$ ) or Section 3 (if $\left.T_{-}\left(U^{1}\right) \in(-\infty, 0)\right)$, there exists $-\theta \in\left(T_{-}\left(U^{1}\right), 0\right)$ such that

$$
\int_{|x| \leq \varepsilon_{3} \theta}\left[\left|\partial_{t} U^{1}(-\theta)\right|^{2}+\left|\nabla U^{1}(-\theta)\right|^{2}\right] \geq \frac{1}{2} \int|\nabla W|^{2} .
$$

Let us show that for large $n$,

$$
\mu\left(\tau_{n}-\theta \mu\left(\tau_{n}\right)\right) \leq \varepsilon_{3} \theta \mu\left(\tau_{n}\right) .
$$


If this holds, by 8.15 we would get

$$
\begin{aligned}
\beta^{2} \theta\left(1-\tau_{n}\right) & \leq \beta \theta \mu\left(\tau_{n}\right) \leq \beta\left(1-\tau_{n}+\theta \mu\left(\tau_{n}\right)\right) \leq \mu\left(\tau_{n}-\theta \mu\left(\tau_{n}\right)\right) \leq \varepsilon_{3} \theta \mu\left(\tau_{n}\right) \\
& \leq \frac{\beta^{2}}{2} \theta\left(1-\tau_{n}\right),
\end{aligned}
$$

a contradiction. The inequality 8.17 is equivalent to

$$
\int_{|x| \leq \varepsilon_{3} \theta \mu\left(\tau_{n}\right)}\left|\nabla_{t, x} a\left(\tau_{n}-\theta \mu\left(\tau_{n}\right)\right)\right|^{2} \geq \frac{2}{5} \int|\nabla W|^{2} .
$$

We have, writing $\theta_{j, n}=-\theta \mu\left(\tau_{n}\right)-t_{j, n}$,

$$
\begin{aligned}
u\left(\tau_{n}-\theta \mu\left(\tau_{n}\right)\right)= & v\left(\tau_{n}-\theta \mu\left(\tau_{n}\right)\right)+\frac{1}{\mu\left(\tau_{n}\right)^{1 / 2}} U^{1}\left(-\theta, \frac{x}{\mu\left(\tau_{n}\right)}\right) \\
& +\sum_{j=2}^{J} \frac{1}{\lambda_{j, n}^{1 / 2}} U^{j}\left(\frac{\theta_{j, n}}{\lambda_{j, n}}, \frac{x}{\lambda_{j, n}}\right)+w_{n}^{J}\left(-\theta \mu\left(\tau_{n}\right)\right)+r_{n}^{J}\left(-\theta \mu\left(\tau_{n}\right)\right), \\
\partial_{t} u\left(\tau_{n}-\theta \mu\left(\tau_{n}\right)\right)= & \partial_{t} v\left(\tau_{n}-\theta \mu\left(\tau_{n}\right)\right)+\frac{1}{\mu\left(\tau_{n}\right)^{3 / 2}} \partial_{t} U^{1}\left(-\theta, \frac{x}{\mu\left(\tau_{n}\right)}\right) \\
& +\sum_{j=2}^{J} \frac{1}{\lambda_{j, n}^{3 / 2}} \partial_{t} U^{j}\left(\frac{\theta_{j, n}}{\lambda_{j, n}}, \frac{x}{\lambda_{j, n}}\right)+\partial_{t} w_{n}^{J}\left(-\theta \mu\left(\tau_{n}\right)\right)+\partial_{t} r_{n}^{J}\left(-\theta \mu\left(\tau_{n}\right)\right),
\end{aligned}
$$

where $r_{n}^{J}$ satisfies

$$
\lim _{J \rightarrow \infty} \limsup _{n \rightarrow \infty}\left[\left\|\nabla r_{n}^{J}\left(\theta \mu\left(\tau_{n}\right)\right)\right\|_{L^{2}}+\left\|\partial_{t} r_{n}^{J}\left(\theta \mu\left(\tau_{n}\right)\right)\right\|_{L^{2}}\right]=0
$$

Let $J_{0}$ be such that for all $J \geq J_{0}$, and for large $n,\left\|\partial_{t, x} r_{n}^{J}\right\|^{2} \leq \frac{1}{40} \int|\nabla W|^{2}$. Let $\psi \in$ $C_{0}^{\infty}\left(\mathbb{R}^{3}\right)$ with $\psi(x)=1$ for $|x| \leq 1$ and $\psi(x)=0$ for $|x| \geq 2$. Then by Remark 2.10 .

$$
\int \psi\left(\frac{x}{\varepsilon_{3} \theta \mu\left(\tau_{n}\right)}\right)\left|\nabla_{t, x} a\left(\tau_{n}-\theta \mu\left(\tau_{n}\right)\right)\right|^{2} \geq \int \psi\left(\frac{x}{\varepsilon_{3} \theta}\right)\left|\nabla_{t, x} U^{1}(-\theta)\right|^{2} \geq \frac{2}{5} \int|\nabla W|^{2},
$$

hence 8.17 holds. The proof is complete.

\subsection{Compactness of the singular part}

Proposition 8.4. Under the assumptions of Theorem 1 (in particular $N=3$ and $u$ is spherically symmetric), a is compact in the energy space up to a scaling parameter: there exists a continuous function $\lambda(t), t \in(0,1)$, such that the closure of

$$
K=\left\{\left(\lambda(t)^{1 / 2} a(t, \lambda(t) x), \lambda(t)^{3 / 2} \partial_{t} a(t, \lambda(t) x)\right): t \in(0,1)\right\}
$$

is compact in $\dot{H}^{1} \times L^{2}$. 
Proof. It is sufficient to show that for any time sequence $\tau_{n} \stackrel{<}{\rightarrow} 1$, there exists a subsequence of $\left\{\tau_{n}\right\}$ and a sequence $\left\{\lambda_{n}\right\}$ such that $\left(\lambda_{n}^{1 / 2} a\left(\tau_{n}, \lambda_{n} x\right), \lambda_{n}^{3 / 2} \partial_{t} a\left(\tau_{n}, \lambda_{n} x\right)\right)$ converges in $\dot{H}^{1} \times L^{2}$.

Let $\tau_{n} \stackrel{<}{\rightarrow}$. After extraction of a subsequence (in $\left.n\right)$, assume that $\left\{\left(a\left(\tau_{n}\right), \partial_{t} a\left(\tau_{n}\right)\right)\right\}$ has a profile decomposition with profiles $\left\{U_{\mathrm{L}}^{j}\right\}$ and parameters $\left\{\lambda_{j, n}, t_{j, n}\right\}$. Let $U^{1}$ be the largest profile. By Lemma 8.1. $\left\|\nabla U_{0}^{1}\right\|_{L^{2}}+\left\|U_{1}^{1}\right\|_{L^{2}} \geq \frac{2}{3}\|\nabla W\|_{L^{2}}^{2}$. By [8.1) and the Pythagorean expansions 2.14) and 2.15, we get

$$
\left\|\nabla w_{n}^{J}\left(\tau_{n}\right)\right\|_{L^{2}}^{2} \leq \frac{1}{3}\|\nabla W\|_{L^{2}}^{2}+\eta_{0}
$$

and

$$
\forall j \geq 2, \quad\left\|\nabla U_{0}^{j}\right\|_{L^{2}}^{2}+\left\|U_{1}^{j}\right\|_{L^{2}}^{2} \leq \frac{1}{3}\|\nabla W\|_{L^{2}}^{2}+\eta_{0} .
$$

This implies that the energies of $U^{j}, j \geq 2$, and of $w_{n}^{J}$ are all positive (see Claim 2.3. We distinguish three cases:

- If $E\left(U^{1}, \partial_{t} U^{1}\right) \geq E(W, 0)$, then by Corollary 8.3 and the Pythagorean expansion of the energy (using that all energies are positive), we see immediately that $E\left(U^{1}, \partial_{t} U^{1}\right)$ $=E(W, 0)$, that there are no other nonzero profiles and that $\left(w_{0, n}^{J}, w_{1, n}^{J}\right)$ tends to 0 as $n \rightarrow \infty$, giving the compactness property.

- If $E\left(U^{1}, \partial_{t} U^{1}\right)<E(W, 0)$, and $\left\|\nabla U_{0}^{1}\right\|_{L^{2}}^{2}+\left\|U_{1}^{1}\right\|_{L^{2}}^{2}<\|\nabla W\|_{L^{2}}^{2}$, the profile $U^{1}$ scatters, yielding immediately a contradiction.

- If $E\left(U^{1}, \partial_{t} U^{1}\right)<E(W, 0)$, and $\left\|\nabla U_{0}^{1}\right\|_{L^{2}}^{2}+\left\|U_{1}^{1}\right\|_{L^{2}}^{2}>\|\nabla W\|_{L^{2}}^{2}$, then the nonlinear solution $U^{1}$ blows up in both time directions. By Proposition 2.8, $U^{1}$ is a type II blow-up solution of (1.1) such that $E\left(U^{1}, \partial_{t} U^{1}\right)<E(W, 0)$. Furthermore, as $\left(a, \partial_{t} a\right)$ converges weakly to 0 and $\left(v, \partial_{t} v\right)$ converges strongly in $\dot{H}^{1} \times L^{2}$ as $t \rightarrow 1$, we have

$$
\int\left|\nabla_{t, x} u(t, x)\right|^{2}=\int\left|\nabla_{t, x} a(t, x)\right|^{2}+\int\left|\nabla_{t, x} v(t, x)\right|^{2}+o(1) \quad \text { as } t \rightarrow 1^{-} .
$$

Thus $U^{1}$ also satisfies 8.1 , which shows that $U^{1}$ contradicts Corollary 8.3

The proof is complete.

\subsection{Convergence to the stationary solution up to scaling}

In this section we conclude the proof of Theorem 1 . Consider a solution $u$ of $(1.1)$ satisfying the assumptions of Theorem 1 . By Corollary 8.3

$$
\lim _{t \rightarrow 1^{-}} E\left(a(t), \partial_{t} a(t)\right)=E(W, 0) .
$$

By Proposition 8.4 there exists $\lambda(t)$ such that the closure of

$$
K=\left\{\left(\lambda(t)^{1 / 2} a(t, \lambda(t) x), \lambda(t)^{3 / 2} \partial_{t} a(t, \lambda(t) x)\right): t \in(0,1)\right\}
$$

is compact in $\dot{H}^{1} \times L^{2}$. The following result is classical in this setting. 
Lemma 8.5. Let $\tau_{n}$ be a sequence that tends to 1 , and such that

$$
\left(\lambda\left(\tau_{n}\right)^{1 / 2} a\left(\tau_{n}, \lambda\left(\tau_{n}\right) x\right), \lambda\left(\tau_{n}\right)^{3 / 2} \partial_{t} a\left(\tau_{n}, \lambda\left(\tau_{n}\right) x\right)\right) \underset{n \rightarrow \infty}{\longrightarrow}\left(U_{0}, U_{1}\right)
$$

in $\dot{H}^{1} \times L^{2}$. Consider the solution $U$ of (1.1) such that

$$
U_{\uparrow t=0}=U_{0}, \quad \partial_{t} U_{\uparrow t=0}=U_{1} .
$$

Then there exists a continuous function $\tilde{\lambda}$ defined on $\left(T_{-}(U), T_{+}(U)\right)$ such that

$$
\tilde{K}=\left\{\left(\begin{array}{c}
\tilde{\lambda}(t)^{1 / 2} U(t, \tilde{\lambda}(t) x) \\
\tilde{\lambda}(t)^{3 / 2} \partial_{t} U(t, \tilde{\lambda}(t) x)
\end{array}\right): t \in\left(T_{-}(U), T_{+}(U)\right)\right\}
$$

has compact closure in $\dot{H}^{1} \times L^{2}$.

Sketch of proof. We have

$$
\begin{aligned}
& u\left(\tau_{n}, x\right)=v\left(\tau_{n}, x\right)+\frac{1}{\lambda\left(\tau_{n}\right)^{1 / 2}} U_{0}\left(\frac{x}{\lambda\left(\tau_{n}\right)}\right)+o_{n}(1) \quad \text { in } \dot{H}^{1}, \\
& \partial_{t} u\left(\tau_{n}, x\right)=\partial_{t} v\left(\tau_{n}, x\right)+\frac{1}{\lambda\left(\tau_{n}\right)^{3 / 2}} U_{1}\left(\frac{x}{\lambda\left(\tau_{n}\right)}\right)+o_{n}(1) \quad \text { in } L^{2} .
\end{aligned}
$$

Let $T \in\left(T_{-}(U), T_{+}(U)\right)$. By Proposition 2.8.

$$
\begin{gathered}
u\left(\tau_{n}+\lambda\left(\tau_{n}\right) T, x\right)=v\left(\tau_{n}+\lambda\left(\tau_{n}\right) T, x\right)+\frac{1}{\lambda\left(\tau_{n}\right)^{1 / 2}} U\left(T, \frac{x}{\lambda\left(\tau_{n}\right)}\right)+o_{n}(1) \quad \text { in } \dot{H}^{1}, \\
\partial_{t} u\left(\tau_{n}+\lambda\left(\tau_{n}\right) T, x\right)=\partial_{t} v\left(\tau_{n}+\lambda\left(\tau_{n}\right) T, x\right)+\frac{1}{\lambda\left(\tau_{n}\right)^{3 / 2}} \partial_{t} U\left(T, \frac{x}{\lambda\left(\tau_{n}\right)}\right)+o_{n}(1) \text { in } L^{2} .
\end{gathered}
$$

Letting $\sigma_{n}=\tau_{n}+\lambda\left(\tau_{n}\right) T$, we get

$$
\begin{aligned}
& \left(\sigma_{n}\right)^{1 / 2} a\left(\sigma_{n}, \lambda\left(\sigma_{n}\right) x\right)=\left(\frac{\lambda\left(\sigma_{n}\right)}{\lambda\left(\tau_{n}\right)}\right)^{1 / 2} U\left(T, \frac{\lambda\left(\sigma_{n}\right)}{\lambda\left(\tau_{n}\right)} x\right)+o_{n}(1) \quad \text { in } \dot{H}^{1}, \\
& \lambda\left(\sigma_{n}\right)^{3 / 2} \partial_{t} a\left(\sigma_{n}, x\right)=\left(\frac{\lambda\left(\sigma_{n}\right)}{\lambda\left(\tau_{n}\right)}\right)^{3 / 2} \partial_{t} U\left(T, \frac{\lambda\left(\sigma_{n}\right)}{\lambda\left(\tau_{n}\right)} x\right)+o_{n}(1) \text { in } L^{2} .
\end{aligned}
$$

Extracting subsequences, we deduce from the compactness of $\bar{K}$ that there exists $\left(V_{0}, V_{1}\right)$ $\in \bar{K}$ such that

$$
\begin{aligned}
\lim _{n \rightarrow \infty}\left(\left(\frac{\lambda\left(\sigma_{n}\right)}{\lambda\left(\tau_{n}\right)}\right)^{1 / 2} U\left(T, \frac{\lambda\left(\sigma_{n}\right)}{\lambda\left(\tau_{n}\right)} x\right),\left(\frac{\lambda\left(\sigma_{n}\right)}{\lambda\left(\tau_{n}\right)}\right)^{3 / 2} \partial_{t} U\left(T, \frac{\lambda\left(\sigma_{n}\right)}{\lambda\left(\tau_{n}\right)} x\right)\right)=\left(V_{0}, V_{1}\right) & \text { in } \dot{H}^{1} \times L^{2} .
\end{aligned}
$$

This shows that $\lambda\left(\sigma_{n}\right) / \lambda\left(\tau_{n}\right)$ has a limit $\tilde{\lambda}(T) \in(0, \infty)$ (by conservation of energy $(0,0) \notin \bar{K})$ and that

$$
\left(\tilde{\lambda}(T)^{1 / 2} U(T, \tilde{\lambda}(T) x), \tilde{\lambda}(T)^{3 / 2} \partial_{t} U(T, \tilde{\lambda}(T) x)\right) \in \bar{K} .
$$


The proof is complete, up to the proof of the known fact that the function $T \mapsto \tilde{\lambda}(T)$ may be taken continuous, for which we refer to [KM06, Remark 5.4].

We next prove Theorem 1

Step 1: Convergence to $W$ for sequences. Let $\left\{t_{n}\right\}_{n}$ be a sequence in $(0,1)$ such that $t_{n} \rightarrow 1$ and

$$
\lim _{n \rightarrow \infty}\left(\lambda\left(t_{n}\right)^{1 / 2} a\left(t_{n}, \lambda\left(t_{n}\right) x\right), \lambda\left(t_{n}\right)^{3 / 2} \partial_{t} a\left(t_{n}, \lambda\left(t_{n}\right) x\right)\right)=\left(U_{0}, U_{1}\right) \quad \text { in } \dot{H}^{1} \times L^{2} .
$$

In this step we show that for some $\lambda_{0}>0$ and some sign + or,$-\left(U_{0}, U_{1}\right)=$ $\pm\left(\lambda_{0}^{1 / 2} W\left(\lambda_{0} \cdot\right), 0\right)$.

Let $U$ be the solution of 1.1 with initial condition $\left(U_{0}, U_{1}\right)$. By Lemma $8.5, U$ is compact up to scaling. By Theorem 2, $U=W$ up to the symmetries, concluding Step 1.

Step 2: Estimate on the scaling parameter. Let

$$
\lambda_{1}(t)=\inf \left\{\mu>0: \int_{|x| \leq \mu}|\nabla u(t, x)-\nabla v(t, x)|^{2} d x \geq \int_{|x| \geq 1}|\nabla W|^{2} d x\right\} .
$$

By Step 1, $\int|\nabla a(t, x)|^{2} d x \rightarrow \int|\nabla W|^{2}$ as $t \rightarrow 1$, which shows that $\lambda_{1}(t)$ is well-defined for $t<1$, close to 1 . Consider a sequence $t_{n} \stackrel{<}{\rightarrow} 1$. By Step 1 , for $\iota_{0}=-1$ or +1 and some sequence $\left\{\lambda_{n}\right\}_{n}$ of positive numbers,

$$
a\left(t_{n}, x\right)=\iota_{0} \frac{1}{\lambda_{n}^{1 / 2}} W\left(\frac{x}{\lambda_{n}}\right)+o_{n}(1) \quad \text { in } \dot{H}^{1} .
$$

Thus if $\mu>0$,

$$
\begin{aligned}
\int_{|x| \leq \mu}\left|\nabla a\left(t_{n}, x\right)\right|^{2} d x & =\int_{|x| \leq \mu} \frac{1}{\lambda_{n}^{3}}\left|\nabla W\left(\frac{x}{\lambda_{n}}\right)\right|^{2}+o_{n}(1) \\
& =\int_{|y| \leq \mu / \lambda_{n}}|\nabla W(y)|^{2} d y+o_{n}(1),
\end{aligned}
$$

which shows that

$$
\lim _{n \rightarrow \infty} \frac{\lambda_{n}}{\lambda_{1}\left(t_{n}\right)}=1
$$

Thus

$$
a\left(t_{n}, x\right)=\iota_{0} \frac{1}{\lambda_{1}\left(t_{n}\right)^{1 / 2}} W\left(\frac{x}{\lambda_{1}\left(t_{n}\right)}\right)+o_{n}(1) \quad \text { in } \dot{H}^{1} .
$$

Step 3: Choice of the sign. Let

$$
f(t)=\int \nabla a(t, x) \cdot \frac{1}{\lambda_{1}(t)^{1 / 2}} \nabla W\left(\frac{x}{\lambda_{1}(t)}\right) d x .
$$

Then by Step 2, for each sequence $t_{n} \stackrel{<}{\rightarrow}$, there exists a subsequence such that $f\left(t_{n}\right) \rightarrow$ $\pm \int|\nabla W|^{2}$. As $f$ is a continuous function, the intermediate value theorem implies that 
the value must be the same for all the sequences $\left\{t_{n}\right\}$. Changing $u$ into $-u$ if necessary, we can assume

$$
\lim _{t \rightarrow 1^{-}} f(t)=\int|\nabla W|^{2}
$$

By Step 2, for all sequences $\left\{t_{n}\right\}$,

$$
u\left(t_{n}, x\right)=v\left(t_{n}, x\right)+\frac{1}{\lambda_{1}\left(t_{n}\right)^{1 / 2}} W\left(\frac{x}{\lambda_{1}\left(t_{n}\right)}\right)+o_{n}(1),
$$

which concludes the proof of the development 1.8 .

Step 4: Estimate on $\lambda_{1}$. Recalling that $u-v$ is supported in the cone $\{|x| \leq 1-t\}$, we get, for $t$ close to 1 ,

$$
\begin{aligned}
0=\int_{|x| \geq 1-t}|\nabla u(t)-\nabla v(t)|^{2} d x & =\int_{|x| \geq 1-t} \frac{1}{\lambda_{1}^{3}(t)}\left|\nabla W\left(\frac{x}{\lambda_{1}(t)}\right)\right|^{2} d x+o(1) \\
& =\int_{|y| \geq \frac{1-t}{\lambda_{1}(t)}}|\nabla W(y)|^{2} d y+o(1)
\end{aligned}
$$

as $t \rightarrow 1^{-}$, which shows that $(1-t) / \lambda_{1}(t) \rightarrow \infty$, concluding the proof of Theorem 1 .

\section{Appendix A. Properties of profiles}

In this appendix we prove a pseudo-orthogonality property (Claim A.1) and Claim 2.11

Claim A.1. Assume that $N \geq 3$ is odd. Let $\left\{w_{n}\right\}$ be a sequence of finite energy solutions of the linear wave equation $\overline{2.1}$, bounded in the energy space, and $U$ be a finite energy solution of 2.1. Consider real sequences $\left\{\lambda_{n}\right\},\left\{\mu_{n}\right\},\left\{t_{n}\right\},\left\{\theta_{n}\right\}$ with $\lambda_{n}, \mu_{n}>0$. Assume that

$$
\lambda_{n}^{N / 2} \nabla_{t, x} w_{n}\left(t_{n}, \lambda_{n} \cdot\right) \underset{n \rightarrow \infty}{\longrightarrow} 0 \quad \text { in } L^{2}\left(\mathbb{R}^{N+1}\right)
$$

Then, if $\varphi=1$, or if $\varphi$ is a radial, continuous, compactly supported function on $\mathbb{R}^{N}$ such that $\varphi(r)=1$ if $r$ is small, there exist subsequences such that

$$
\lim _{n \rightarrow \infty} \int \varphi\left(\frac{|x|}{\mu_{n}}\right) \nabla_{t, x} w_{n}\left(\theta_{n}, x\right) \cdot \frac{1}{\lambda_{n}^{N / 2}} \nabla_{t, x} U\left(\frac{\theta_{n}-t_{n}}{\lambda_{n}}, \frac{x}{\lambda_{n}}\right) d x=0
$$

and

$$
\lim _{n \rightarrow \infty} \int\left(1-\varphi\left(\frac{|x|}{\mu_{n}}\right)\right) \nabla_{t, x} w_{n}\left(\theta_{n}, x\right) \cdot \frac{1}{\lambda_{n}^{N / 2}} \nabla_{t, x} U\left(\frac{\theta_{n}-t_{n}}{\lambda_{n}}, \frac{x}{\lambda_{n}}\right) d x=0 .
$$


Proof. We start by showing A.2 when $\varphi=1$. By conservation of energy for solutions of (2.1),

$$
\begin{aligned}
\int \nabla_{t, x} w_{n}\left(\theta_{n}, x\right) \frac{1}{\lambda_{n}^{N / 2}} \nabla_{t, x} U\left(\frac{\theta_{n}-t_{n}}{\lambda_{n}}\right. & \left., \frac{x}{\lambda_{n}}\right) d x \\
& =\int \nabla_{t, x} w_{n}\left(t_{n}, x\right) \frac{1}{\lambda_{n}^{N / 2}} \nabla_{t, x} U\left(0, \frac{x}{\lambda_{n}}\right) d x
\end{aligned}
$$

By the change of variable $\lambda_{n} y=x$, we see that A.1 implies A.2 for $\varphi=1$.

We next consider the case when $\varphi \in C^{0}\left(\mathbb{R}^{N}\right)$ is compactly supported and satisfies $\varphi=1$ around 0 . Because of the case $\varphi=1$, one of the estimates A.2 or A.3 implies the other. By the change of variable $\mu_{n} y=x$,

$$
\begin{aligned}
\int \varphi\left(\frac{|x|}{\mu_{n}}\right) & \nabla_{t, x} w_{n}\left(\theta_{n}, x\right) \cdot \frac{1}{\lambda_{n}^{N / 2}} \nabla_{t, x} U\left(\frac{\theta_{n}-t_{n}}{\lambda_{n}}, \frac{x}{\lambda_{n}}\right) d x \\
& =\int \varphi(|y|) \mu_{n}^{N / 2} \nabla_{t, x} w_{n}\left(\mu_{n} \tilde{\theta}_{n}, \mu_{n} y\right) \cdot \frac{1}{\tilde{\lambda}_{n}^{N / 2}} \nabla_{t, x} U\left(\frac{\tilde{\theta}_{n}-\tilde{t}_{n}}{\tilde{\lambda}_{n}}, \frac{y}{\tilde{\lambda}_{n}}\right) d y
\end{aligned}
$$

where $\tilde{\theta}_{n}=\theta_{n} / \mu_{n}, \tilde{\lambda}_{n}=\lambda_{n} / \mu_{n}, \tilde{t}_{n}=t_{n} / \mu_{n}$. Replacing $w_{n}$ by the solution $(t, y) \mapsto$ $\mu_{n}^{N / 2} w_{n}\left(\mu_{n} t, \mu_{n} y\right)$ of 2.1], $\theta_{n}$ by $\tilde{\theta}_{n}, t_{n}$ by $\tilde{t}_{n}$ and $\lambda_{n}$ by $\tilde{\lambda}_{n}$, we will assume, in addition to A.1., that $\mu_{n}=1$ for all $n$.

Extracting subsequences, we distinguish two cases.

Case 1. Assume

$$
\lim _{n \rightarrow \infty} \frac{\theta_{n}-t_{n}}{\lambda_{n}}= \pm \infty
$$

Then, by Lemma 4.1 , the energy of $\frac{1}{\lambda_{n}^{N / 2}} U\left(\frac{\theta_{n}-t_{n}}{\lambda_{n}}, \frac{x}{\lambda_{n}}\right)$ concentrates in sets of the form

$$
\left\{\left|\theta_{n}-t_{n}\right|-C \lambda_{n} \leq|x| \leq\left|\theta_{n}-t_{n}\right|+C \lambda_{n}\right\}
$$

Recalling that $\mu_{n}=1$, we deduce that if $\left|\theta_{n}-t_{n}\right| \rightarrow \infty$, then A.2 holds, and if $\left|\theta_{n}-t_{n}\right| \rightarrow 0$, then $\mathrm{A} .3$ holds. In both cases, the proof is complete.

We next assume, after extraction, that

$$
\lim _{n \rightarrow \infty}\left(\theta_{n}-t_{n}\right)=T \in \mathbb{R}^{*}
$$

Let $\varepsilon>0$, and let $R$ (given by Lemma 4.1 be such that

$$
\limsup _{n \rightarrow \infty} \int_{\mathcal{C C}_{n}(R)} \frac{1}{\lambda_{n}^{N}}\left|\nabla_{t, x} U\left(\frac{\theta_{n}-t_{n}}{\lambda_{n}}, \frac{x}{\lambda_{n}}\right)\right|^{2} d x \leq \varepsilon^{2},
$$


where $\mathcal{C}_{n}(R)=\left\{x \in \mathbb{R}^{N}:\left|\theta_{n}-t_{n}\right|-R \lambda_{n} \leq|x| \leq\left|\theta_{n}-t_{n}\right|+R \lambda_{n}\right\}$ and $C \mathcal{C}_{n}(R)$ is its complement in $\mathbb{R}^{N}$. Using the boundedness of $\nabla_{t, x} w_{n}$ in $\left(L^{2}\right)^{N+1}$, we get, for large $n$,

$$
\begin{aligned}
\mid \int\left(\varphi(x)-\varphi\left(\left|\theta_{n}-t_{n}\right|\right)\right) \nabla_{t, x} w_{n}\left(\theta_{n}, x\right) & \frac{1}{\lambda_{n}^{N / 2}} \nabla_{t, x} U\left(\frac{\theta_{n}-t_{n}}{\lambda_{n}}, \frac{x}{\lambda_{n}}\right) d x \mid \\
& \leq C \max _{x \in \mathcal{C}_{n}(R)}\left|\varphi(x)-\varphi\left(\left|\theta_{n}-t_{n}\right|\right)\right|+C \varepsilon
\end{aligned}
$$

where the constant $C$ depends only on the energy of $U$ and the bound of $\nabla_{t, x} w_{n}$ in $\left(L^{2}\right)^{N+1}$. As $\varphi$ is uniformly continuous, and $\lambda_{n} \rightarrow 0$, by A.4 and A.5 we get

$$
\limsup _{n \rightarrow \infty}\left|\int\left(\varphi(x)-\varphi\left(\left|\theta_{n}-t_{n}\right|\right)\right) \nabla_{t, x} w_{n}\left(\theta_{n}, x\right) \frac{1}{\lambda_{n}^{N / 2}} \nabla_{t, x} U\left(\frac{\theta_{n}-t_{n}}{\lambda_{n}}, \frac{x}{\lambda_{n}}\right) d x\right| \leq C \varepsilon,
$$

and hence (using the case $n=1$ ),

$$
\limsup _{n \rightarrow \infty}\left|\int \varphi(x) \nabla_{t, x} w_{n}\left(\theta_{n}, x\right) \frac{1}{\lambda_{n}^{N / 2}} \nabla_{t, x} U\left(\frac{\theta_{n}-t_{n}}{\lambda_{n}}, \frac{x}{\lambda_{n}}\right) d x\right| \leq C \varepsilon .
$$

The proof is complete if A.4 holds.

Case 2. Assume

$$
\lim _{n \rightarrow \infty} \frac{\theta_{n}-t_{n}}{\lambda_{n}}=t_{0} \in \mathbb{R}
$$

Then by Lemma 4.1 the $L^{2}$ norm of $\frac{1}{\lambda_{n}^{N / 2}} \nabla_{t, x} U\left(\frac{\theta_{n}-t_{n}}{\lambda_{n}}, \frac{x}{\lambda_{n}}\right)$ is localized in sets of the form

$$
\left\{C^{-1} \lambda_{n} \leq|x| \leq C \lambda_{n}\right\}
$$

If $\lambda_{n} \rightarrow \infty$ or $\lambda_{n} \rightarrow 0$, the argument of Case 1 yields A.2 and A.3. Let us assume

$$
\lim _{n \rightarrow \infty} \lambda_{n}=\lambda_{\infty} \in(0, \infty)
$$

Then

$$
\frac{1}{\lambda_{n}^{N / 2}} \nabla_{t, x} U\left(\frac{\theta_{n}-t_{n}}{\lambda_{n}}, \frac{x}{\lambda_{n}}\right)=\frac{1}{\lambda_{\infty}^{N / 2}} \nabla_{t, x} U\left(t_{0}, \frac{x}{\lambda_{\infty}}\right)+o_{n}(1) \quad \text { in }\left(L^{2}\left(\mathbb{R}^{N}\right)\right)^{N+1} .
$$

Thus we must show

$$
\lim _{n \rightarrow \infty} \int \varphi(x) \nabla_{t, x} w_{n}\left(\theta_{n}, x\right) \frac{1}{\lambda_{\infty}^{N / 2}} \nabla_{t, x} U\left(t_{0}, \frac{x}{\lambda_{\infty}}\right) d x=0 .
$$

First notice that if $\Phi \in\left(L^{2}\right)^{N+1}$,

$$
\begin{aligned}
\int \nabla_{t, x} w_{n}\left(t_{n}, x\right) \cdot \Phi(x) d x & =\int \lambda_{n}^{N / 2} \nabla_{t, x} w_{n}\left(t_{n}, \lambda_{n} y\right) \cdot \lambda_{n}^{N / 2} \Phi\left(\lambda_{n} y\right) d y \\
& =\int \lambda_{n}^{N / 2} \nabla_{t, x} w_{n}\left(t_{n}, \lambda_{n} y\right) \cdot \lambda_{\infty}^{N / 2} \Phi\left(\lambda_{\infty} y\right) d y+o_{n}(1)
\end{aligned}
$$


At the last line we used that $\lambda_{n}^{N / 2} \Phi\left(\lambda_{n} y\right)$ converges strongly to $\lambda_{\infty}^{N / 2} \Phi\left(\lambda_{\infty} y\right)$ in $\left(L^{2}\right)^{N+1}$. Thus by (A.1,

$$
\nabla_{t, x} w_{n}\left(t_{n}, x\right) \underset{n \rightarrow \infty}{\longrightarrow} 0 \quad \text { in }\left(L^{2}\right)^{N+1}
$$

Next, consider the solution $v$ of 2.1 with initial data $\left(v_{0}, v_{1}\right) \in \dot{H}^{1} \times L^{2}$ such that

$$
\Delta v_{0}(x)=\frac{1}{\lambda_{\infty}^{N / 2}} \operatorname{div}\left(\varphi(x) \nabla_{x} U\left(t_{0}, \frac{x}{\lambda_{\infty}}\right)\right), \quad v_{1}(x)=\frac{1}{\lambda_{\infty}^{N / 2}} \varphi(x) \partial_{t} U\left(t_{0}, \frac{x}{\lambda_{\infty}}\right) .
$$

Write $\theta_{n}=\lambda_{\infty} t_{0}+t_{n}+\varepsilon_{n}$ with $\varepsilon_{n} \rightarrow 0^{+}$. Then by conservation of energy,

$$
\begin{aligned}
\int \varphi(x) \nabla_{t, x} w_{n}\left(\theta_{n}, x\right) \frac{1}{\lambda_{\infty}^{N / 2}} \nabla_{t, x} U\left(t_{0},\right. & \left.\frac{x}{\lambda_{\infty}}\right) d x=\int \nabla_{t, x} w_{n}\left(\theta_{n}, x\right) \nabla_{t, x} v(0, x) d x \\
& =\int \nabla_{t, x} w_{n}\left(t_{n}, x\right) \nabla_{t, x} v\left(-\lambda_{\infty} t_{0}-\varepsilon_{n}, x\right) d x \\
& =\int \nabla_{t, x} w_{n}\left(t_{n}, x\right) \nabla_{t, x} v\left(-\lambda_{\infty} t_{0}, x\right) d x+o_{n}(1)
\end{aligned}
$$

which shows A.7 in view of A.8.

We next prove Claim 2.11

Proof of Claim 2.11 We prove the result when $N$ is odd, although it should also hold when $N$ is even. Rescaling if necessary, we will assume

$$
\forall n, \quad \tilde{\lambda}_{n}=1
$$

Note that the assumption implies that for any sequences $\left\{\lambda_{n}\right\},\left\{t_{n}\right\}$,

$\left(\frac{1}{\lambda_{n}^{(N-2) / 2}} w_{n}\left(\frac{-t_{n}}{\lambda_{n}}, \frac{\cdot}{\lambda_{n}}\right), \frac{1}{\lambda_{n}^{N / 2}} \partial_{t} w_{n}\left(\frac{-t_{n}}{\lambda_{n}}, \frac{\cdot}{\lambda_{n}}\right)\right) \underset{n \rightarrow \infty}{\underset{ }{\longrightarrow}}(0,0) \quad$ weakly in $\dot{H}^{1} \times L^{2}$.

Indeed, if A.10 does not hold, the sequence $\left\{w_{n}\right\}$ would have a nontrivial profile decomposition, contradicting 2.26.

Conversely, we claim that 2.27 holds as soon as for all sequences $\left\{\lambda_{n}\right\},\left\{t_{n}\right\}$,

$\left(\frac{1}{\lambda_{n}^{(N-2) / 2}} \tilde{w}_{n}\left(\frac{-t_{n}}{\lambda_{n}}, \frac{\cdot}{\lambda_{n}}\right), \frac{1}{\lambda_{n}^{N / 2}} \partial_{t} \tilde{w}_{n}\left(\frac{-t_{n}}{\lambda_{n}}, \frac{\cdot}{\lambda_{n}}\right)\right) \underset{n \rightarrow \infty}{\longrightarrow}(0,0) \quad$ weakly in $\dot{H}^{1} \times L^{2}$.

Again, if 2.27) does not hold, then the sequence $\left(\tilde{w}_{n}(0), \partial_{t} \tilde{w}_{n}(0)\right)$ has a profile decomposition with at least one nonzero profile, which contradicts A.11. 
Let us show A.11. Let $\left(Z_{0}, V_{1}\right) \in \dot{H}^{-1} \times L^{2}$ and let $V_{0} \in \dot{H}^{1}$ be such that $\Delta V_{0}$ $=Z_{0}$. Let $V$ be the solution of (2.1) with initial conditions $\left(V_{0}, V_{1}\right)$. We have

$$
\begin{aligned}
& \int \frac{1}{\lambda_{n}^{(N-2) / 2}} \tilde{w}_{n}\left(\frac{-t_{n}}{\lambda_{n}}, \frac{x}{\lambda_{n}}\right) Z_{0}(x) d x+\int \frac{1}{\lambda_{n}^{N / 2}} \partial_{t} \tilde{w}_{n}\left(\frac{-t_{n}}{\lambda_{n}}, \frac{x}{\lambda_{n}}\right) V_{1}(x) d x \\
& =\int \nabla_{x} \tilde{w}_{n}(0, x) \cdot \lambda_{n}^{N / 2} \nabla_{x} V\left(t_{n}, \lambda_{n} x\right) d x+\int \partial_{t} \tilde{w}_{n}(0, x) \lambda_{n}^{N / 2} \partial_{t} V\left(t_{n}, \lambda_{n} x\right) \\
& =\int \nabla_{x}\left(\varphi(|x|) w_{0, n}(x)\right) \cdot \lambda_{n}^{N / 2} \nabla_{x} V\left(t_{n}, \lambda_{n} x\right) d x+\int \varphi(|x|) w_{1, n}(x) \lambda_{n}^{N / 2} \partial_{t} V\left(t_{n}, \lambda_{n} x\right) .
\end{aligned}
$$

Thus it suffices to show

$$
\begin{aligned}
& \lim _{n \rightarrow \infty} \int \varphi(|x|) \nabla_{x} w_{0, n}(x) \cdot \lambda_{n}^{N / 2} \nabla_{x} V\left(t_{n}, \lambda_{n} x\right) d x \\
& +\int \varphi(|x|) w_{1, n}(x) \lambda_{n}^{N / 2} \partial_{t} V\left(t_{n}, \lambda_{n} x\right)=0, \\
& \lim _{n \rightarrow \infty} \int\left(\nabla_{x} \varphi(|x|)\right) w_{0, n}(x) \cdot \lambda_{n}^{N / 2} \nabla_{x} V\left(t_{n}, \lambda_{n} x\right) d x=0 .
\end{aligned}
$$

The first limit, $\mathrm{A.13}$, follows immediately from Claim A.1 To show $(\mathrm{A})$, we use that there exists $C>0$ such that $\nabla \varphi$ is supported in $\{1 / C \leq|x| \leq C\}$, and distinguish several cases.

If $t_{n}$ is bounded, then one can assume after extraction that $t_{n}$ has a limit $T \in[0, \infty)$. If $\lambda_{n} \rightarrow 0$ or $\lambda_{n} \rightarrow \infty$ then by Lemma 4.1 .

$$
\lim _{n \rightarrow \infty} \int_{1 / C \leq|x| \leq C} \lambda_{n}^{N}\left|\nabla V\left(t_{n}, \lambda_{n} x\right)\right|^{2} d x=0,
$$

and Af follows. If $\lambda_{n}$ has a limit $\lambda_{\infty} \in(0, \infty)$, then $\lambda_{n}^{N / 2} \nabla V\left(t_{n}, \lambda_{n} x\right)$ converges strongly to $\lambda_{\infty}^{N / 2} \nabla V\left(T, \lambda_{\infty} x\right)$, and we are reduced to showing

$$
\lim _{n \rightarrow \infty} \int_{1 / C \leq|x| \leq C}\left(\nabla_{x} \varphi(|x|)\right) w_{0, n}(x) \cdot \lambda_{\infty}^{N / 2} \nabla V\left(T, \lambda_{\infty} x\right) d x=0,
$$

which follows from the fact that by A.10, $w_{0, n}$ tends to 0 weakly in $\dot{H}^{1}$ (and thus, by Hardy's inequality, $|x|^{-1} w_{0, n}$ tends to 0 weakly in $L^{2}$ ).

We next treat the case when $t_{n}$ is not bounded. Extracting, we assume that $t_{n} \rightarrow \infty$ (the case $t_{n} \rightarrow-\infty$ is analogous). If $t_{n} / \lambda_{n} \rightarrow 0$ or $t_{n} / \lambda_{n} \rightarrow \infty$, Lemma 4.1 implies again (A.15), and A follows. It remains to consider the case when (after extraction) $t_{n} / \lambda_{n} \rightarrow \ell \in(0, \infty)$. By Lemma 4.1. for all $\varepsilon>0$ there exists $R_{\varepsilon}$ such that for all $R \geq R_{\varepsilon}$,

$$
\limsup _{n \rightarrow \infty} \int_{|| x|-\ell| \geq R / \lambda_{n}} \lambda_{n}^{N}\left|\nabla V\left(t_{n}, \lambda_{n} x\right)\right|^{2} d x \leq \varepsilon
$$


As a consequence,

$$
\limsup _{n \rightarrow \infty} \int_{|| x|-\ell| \geq 1 / \sqrt{\lambda_{n}}} \lambda_{n}^{N}\left|\nabla V\left(t_{n}, \lambda_{n} x\right)\right|^{2} d x=0 .
$$

It remains to show that

$$
\lim _{n \rightarrow \infty}\left|\int_{|| x|-\ell| \leq 1 / \sqrt{\lambda_{n}}} \partial_{r} \varphi(|x|) w_{0, n}(x) \lambda_{n}^{N / 2} \partial_{r} V\left(t_{n}, \lambda_{n} x\right) d x\right|=0 .
$$

We have

$$
\begin{aligned}
\int_{|| x|-\ell| \leq 1 / \sqrt{\lambda_{n}}} \partial_{r} \varphi(|x|) w_{0, n}(x) \lambda_{n}^{N / 2} \partial_{r} V\left(t_{n}, \lambda_{n} x\right) d x \\
=\int_{|| x|-\ell| \leq 1 / \sqrt{\lambda_{n}}}|x| \partial_{r} \varphi(|x|) \frac{1}{|x|} w_{0, n}(x) \lambda_{n}^{N / 2} \partial_{r} V\left(t_{n}, \lambda_{n} x\right) d x \\
=\int_{|| x|-\ell| \leq 1 / \sqrt{\lambda_{n}}} \ell \partial_{r} \varphi(\ell) \frac{1}{|x|} w_{0, n}(x) \lambda_{n}^{N / 2} \partial_{r} V\left(t_{n}, \lambda_{n} x\right) d x+o_{n}(1) \\
=\ell \partial_{r} \varphi(\ell) \int_{\mathbb{R}^{N}} \frac{1}{|x|} w_{0, n}(x) \lambda_{n}^{N / 2} \partial_{r} V\left(t_{n}, \lambda_{n} x\right) d x+o_{n}(1) .
\end{aligned}
$$

At the third line, we have used that $r \partial_{r} \varphi$ is continuous and thus

$$
\lim _{n \rightarrow \infty} \sup _{|r-\ell| \leq 1 / \sqrt{\lambda_{n}}}\left|r \partial_{r} \varphi(r)-\ell \partial_{r} \varphi(\ell)\right|=0 .
$$

At the last line we have used A.16. By Hardy's inequality and assumption (A.10), $|x|^{-1} w_{0, n}$ converges weakly to 0 in $L^{2}$, and thus (A.18) implies (A.17), which concludes the proof of Claim 2.11 .

\section{Appendix B. Family of sequences of positive numbers}

Claim B.1. Let $\left\{\lambda_{n}\right\}_{n},\left\{v_{n}\right\}_{n}$ and for $j \in \mathbb{N},\left\{\rho_{j, n}\right\}_{n}$, be sequences of positive numbers and assume

$$
\lambda_{n} \ll v_{n} .
$$

Then, after extraction of subsequences, there exists a sequence $\left\{\mu_{n}\right\}_{n}$ such that

$$
\begin{aligned}
\lambda_{n} & \ll \mu_{n} \ll v_{n}, \\
\forall k, \quad \mu_{n} & \ll \rho_{k, n} \text { or } \rho_{k, n} \ll \mu_{n} .
\end{aligned}
$$

Proof. Let, for $s \in(0,1)$,

$$
\mu_{n}(s)=\lambda_{n}^{1-s} v_{n}^{s}
$$


Note that for any $s \in(0,1), \lambda_{n} \ll \mu_{n}(s) \ll v_{n}$. Let $j \in \mathbb{N}$. Then, extracting subsequences in $n$ if necessary, we are in one of the following three cases:

$$
\left\{\begin{array}{l}
\forall s \in(0,1), \quad \mu_{n}(s) \ll \rho_{j, n} \text { or } \\
\forall s \in(0,1), \quad \rho_{j, n} \ll \mu_{n}(s) \text { or } \\
\exists s_{j} \in(0,1), \quad \forall s \in\left(0, s_{j}\right), \mu_{n}(s) \ll \rho_{j, n} \text { and } \forall s \in\left(s_{j}, 1\right), \rho_{j, n} \ll \mu_{n}(s) .
\end{array}\right.
$$

Indeed, let

$$
s_{j}=\inf \left\{s \in[0,1]:\left\{\rho_{j, n} / \mu_{n}(s)\right\}_{n} \text { is bounded. }\right\} .
$$

Note that $\mu_{n}(s) \ll \mu_{n}\left(s^{\prime}\right)$ is $s<s^{\prime}$. As a consequence, if $s_{j}=0$, then $\rho_{j, n} / \mu_{n}(s) \rightarrow 0$ for all $s \in(0,1)$. Similarly if $s_{j}=1$, then $\left\{\rho_{j, n} / \mu_{n}(s)\right\}_{n}$ is never bounded for $s \in(0,1)$ and by diagonal extraction we can find a subsequence such that $\rho_{j, n} / \mu_{n}(s) \rightarrow \infty$ for any $s \in(0,1)$. Finally if $s_{j} \in(0,1)$, then $\rho_{j, n} / \mu_{n}(s) \rightarrow 0$ for all $s \in\left(s_{j}, 1\right)$, and $\left\{\rho_{j, n} / \mu_{n}(s)\right\}_{n}$ is not bounded for $s \in\left(0, s_{j}\right)$. Using diagonal extraction again we can assume that $\rho_{j, n} / \mu_{n}(s) \rightarrow \infty$ for all $s \in\left(0, s_{j}\right)$. Hence $(\mathrm{B} .4)$ follows.

After another diagonal extraction, we can assume that (B.4) holds for all $j \in \mathbb{N}$. Choosing $s \in(0,1)$ distinct from all $s_{j}$, and letting $\mu_{n}=\mu_{n}(s)$, we get the desired properties (B.2) and (B.3).

Acknowledgments. Research of T. Duyckaerts was partially supported by ANR Grants ONDNONLIN and ControlFlux. Research of C. Kenig was partially supported by NSF Grant DMS-0456583. Research of F. Merle was partially supported by ANR Grant ONDNONLIN.

\section{References}

[Aub76] Aubin, T.: Équations différentielles non linéaires et problème de Yamabe concernant la courbure scalaire. J. Math. Pures Appl. (9) 55, 269-296 (1976) Zbl 0336.53033 MR 0431287

[BG99] Bahouri, H., Gérard, P.: High frequency approximation of solutions to critical nonlinear wave equations. Amer. J. Math. 121, 131-175 (1999) Zbl 0919.35089 MR 1705001

[BC85] Brezis, H., Coron, J.-M.: Convergence of solutions of $H$-systems or how to blow bubbles. Arch. Ration. Mech. Anal. 89, 21-56 (1985) Zbl 0584.49024 MR 0784102

[CF86] Caffarelli, L. A., Friedman, A.: The blow-up boundary for nonlinear wave equations. Trans. Amer. Math. Soc. 297, 223-241 (1986) Zbl $0638.35053 \mid$ MR 0849476

[CTZ93] Christodoulou, D., Tahvildar-Zadeh, A. S.: On the asymptotic behavior of spherically symmetric wave maps. Duke Math. J. 71, 31-69 (1993) Zbl 0791.58105 MR 1230285

[DKM] Duyckaerts, T., Kenig, C., Merle, F.: Universality of the blow-up profile for small type II blow-up solutions of the energy-critical wave equation: the non-radial case. J. Eur. Math. Soc., to appear; arXiv:1003.0625 (2010)

[DM08] Duyckaerts, T., Merle, F.: Dynamics of threshold solutions for energy-critical wave equation. Int. Math. Res. Pap. 2008, art. ID rpn002, 67 pp. Zbl 1159.35043 MR 2470571

[GSV92] Ginibre, J., Soffer, A., Velo, G.: The global Cauchy problem for the critical nonlinear wave equation. J. Funct. Anal. 110, 96-130 (1992) Zbl 0813.35054 MR 1190421

[Kap94] Kapitanski, L.: Global and unique weak solutions of nonlinear wave equations. Math. Res. Lett. 1, 211-223 (1994) Zbl 0841.35067| MR 1266760 
[KM06] Kenig, C. E., Merle, F.: Global well-posedness, scattering and blow-up for the energycritical, focusing, non-linear Schrödinger equation in the radial case. Invent. Math. 166, 645-675 (2006) Zbl 1115.35125 MR 2257393

[KM08] Kenig, C. E., Merle, F.: Global well-posedness, scattering and blow-up for the energy-critical focusing non-linear wave equation. Acta Math. 201, 147-212 (2008) Zbl 1183.35202 MR 2461508

[KS07] Krieger, J., Schlag, W.: On the focusing critical semi-linear wave equation. Amer. J. Math. 129, 843-913 (2007) Zbl pre05170329 MR 2325106

[KST08] Krieger, J., Schlag, W., Tataru, D.: Renormalization and blow up for charge one equivariant critical wave maps. Invent. Math. 171, 543-615 (2008) Zbl 1139.35021 MR 2372807

[KST09] Krieger, J., Schlag, W., Tataru, D.: Slow blow-up solutions for the $H^{1}\left(\mathbb{R}^{3}\right)$ critical focusing semilinear wave equation. Duke Math. J. 147, 1-53 (2009) Zbl 1170.35066 MR 2494455

[Lev74] Levine, H. A.: Instability and nonexistence of global solutions to nonlinear wave equations of the form $P u_{t t}=-A u+\mathcal{F}(u)$. Trans. Amer. Math. Soc. 192, 1-21 (1974) Zbl 0288.35003 MR 0344697

[LS95] Lindblad, H., Sogge, C. D.: On existence and scattering with minimal regularity for semilinear wave equations. J. Funct. Anal. 130, 357-426 (1995) Zbl 0846.35085 MR 1335386

[Lio85] Lions, P.-L.: The concentration-compactness principle in the calculus of variations. The limit case. II. Rev. Mat. Iberoamer. 1, no. 2, 45-121 (1985) Zbl 0704.49006 MR 0850686

[MM00] Martel, Y., Merle, F.: A Liouville theorem for the critical generalized Korteweg-de Vries equation. J. Math. Pures Appl. (9) 79, 339-425 (2000) Zbl 0963.37058 MR 1753061

[MM01] Martel, Y., Merle, F.: Asymptotic stability of solitons for subcritical generalized KdV equations. Arch. Ration. Mech. Anal. 157, 219-254 (2001) Zbl 0981.35073 MR 1826966

[MM02] Martel, Y., Merle, F.: Stability of blow-up profile and lower bounds for blow-up rate for the critical generalized KdV equation. Ann. of Math. (2) 155, 235-280 (2002) Zbl 1005.35081 MR 1888800

[MR04] Merle, F., Raphaël, P.: On universality of blow-up profile for $L^{2}$ critical nonlinear Schrödinger equation. Invent. Math. 156, 565-672 (2004) Zbl 1067.35110 MR 2061329

[MR05] Merle, F., Raphaël, P.: Profiles and quantization of the blow up mass for critical nonlinear Schrödinger equation. Comm. Math. Phys. 253, 675-704 (2005) Zbl 1062.35137 MR 2116733

[MR08] Merle, F., Raphaël, P.: Blow up of the critical norm for some radial $L^{2}$ super critical nonlinear Schrödinger equations. Amer. J. Math. 130, 945-978 (2008) Zbl 1188.35182 MR 2427005

[MV98] Merle, F., Vega, L.: Compactness at blow-up time for $L^{2}$ solutions of the critical nonlinear Schrödinger equation in 2D. Int. Math. Res. Notices 1998, no. 8, 399-425 Zbl 0913.35126 MR 1628235

[MZ07] Merle, F., Zaag, H.: Existence and universality of the blow-up profile for the semilinear wave equation in one space dimension. J. Funct. Anal. 253, 43-121 (2007) Zbl 1133.35070 MR 2362418

[MZ08] Merle, F., Zaag, H.: Existence and characterization of characteristic points for a semilinear wave equation in one space dimension. http://arxiv.org/abs/0811.4068 (2008)

[Pec84] Pecher, H.: Nonlinear small data scattering for the wave and Klein-Gordon equation. Math. Z. 185, 261-270 (1984) Zbl 0538.35063 MR 0731347 
[RR] Raphaël, P., Rodnianski, I.: Personal communication

[RS] Rodnianski, I., Sterbenz, J.: On the formation of singularities in the critical $\mathrm{O}(3) \sigma$-model. Ann. of Math. 172, 187-242 (2010) Zbl pre05779357| MR 2680419

[SS94] Shatah, J., Struwe, M.: Well-posedness in the energy space for semilinear wave equations with critical growth. Int. Math. Res. Notices 1994, no. 7, 303ff., approx. 7 pp. Zbl 0830.35086 MR 1283026

[SS98] Shatah, J., Struwe, M.: Geometric Wave Equations. Courant Lecture Notes Math. 2, New York Univ. Courant Inst. of Math. Sci., New York (1998) Zbl 0993.35001 MR 1674843

[STZ97] Shatah, J., Tahvildar-Zadeh, A. S.: On the stability of stationary wave maps. Comm. Math. Phys. 185, 231-256 (1997) Zbl 0877.58054 MR 1463041

[Sog95] Sogge, C. D.: Lectures on Nonlinear Wave Equations. Monogr. Anal. 2, Int. Press, Boston, MA (1995) Zbl 1089.35500 MR 1715192

[ST10] Sterbenz, J., Tataru, D.: Regularity of wave-maps in dimension 2+1. Comm. Math. Phys. 298, 231-264 (2010) Zbl pre05764635 MR 2657818

[Str02] Struwe, M.: Radially symmetric wave maps from $(1+2)$-dimensional Minkowski space to the sphere. Math. Z. 242, 407-414 (2002) Zbl 1052.58034 MR 1985457

[Str03] Struwe, M.: Radially symmetric wave maps from $(1+2)$-dimensional Minkowski space to general targets. Calc. Var. Partial Differential Equations 16, 431-437 (2003) Zbl 1039.58033 MR 1971037

[Tal76] Talenti, G.: Best constant in Sobolev inequality. Ann. Mat. Pura Appl. (4) 110, 353-372 (1976) Zbl 0353.46018 MR 0463908 